Estabilidade de ondas viajantes para equações de Schrödinger do tipo cúbica-quíntica

César Adolfo Hernández Melo

Tese apresentada ao Instituto de Matemática e Estatística da Universidade de São Paulo para obtenção do título de Doutor em Ciências

Programa: Matemática Orientador: Prof. Dr. Jaime Angulo Pava

Durante o desenvolvimento deste trabalho o autor recebeu auxílio financeiro da CNPq São Paulo, janeiro de 2012 



\section{Estabilidade de ondas viajantes para equações de Schrödinger do tipo cúbica-quíntica}

Esta tese trata-se da versão original do aluno César Adolfo Hernández Melo. 



\title{
Estabilidade de ondas viajantes para equações de Schrödinger do tipo cúbica-quíntica
}

\author{
Esta tese contém as correções e alterações \\ sugeridas pela Comissão Julgadora durante a defesa \\ realizada por César Adolfo Hernández Melo em 18/11/2011. \\ O original encontra-se disponível no Instituto de \\ Matemática e Estatística da Universidade de São Paulo.
}

Comissão Julgadora:

- Prof. Dr. Jaime Angulo Pava - IME-USP

- Prof. Dr. Luiz Augusto Fernandes de Oliveira - IME-USP

- Prof. Dr. Arnaldo Simal do Nascimento - UFSCAR

- Prof. Dr. Gustavo Alberto Perla Menzala - LNCC

- Prof. Dr. José Felipe Linares Ramirez - IMPA 



\title{
Resumo
}

\author{
Estabilidade de ondas viajantes \\ para equações de Schrödinger \\ do tipo cúbica-quíntica
}

Este trabalho é dedicado a entender alguns aspectos matemáticos dos seguintes modelos não lineares: a equação de Schrödinger não linear com potência dupla, isto é

$$
i u_{t}+u_{x x}+u|u|^{2}+u|u|^{4}=0
$$

e uma perturbação de tipo delta deste modelo, à saber,

$$
i u_{t}+u_{x x}+Z \delta(x) u+u|u|^{2}+u|u|^{4}=0
$$

Para o primeiro modelo em (1), usando a teoria de integrais elípticas de Jacobi e o teorema da função implícita, obtemos uma família de ondas estacionárias $u(x, t)=$ $e^{-i w t} \phi_{w}(x)$, onde $\phi_{w}: \mathbb{R} \rightarrow \mathbb{R}$ é uma função positiva e periódica de período $L>0$, conhecida como o perfil da onda. Para $L \rightarrow \infty$, mostramos que as ondas estacionárias periódicas tendem uniformemente sobre intervalos compactos à onda solitária. Usando uma extensão da teoria de Angulo\&Natali assim como as idéias desenvolvidas por Weinstein, Bona, Grillakis, Shatah e Strauss, mostramos estabilidade orbital desas ondas por perturbações do mesmo período que a onda. Por fim, provamos um resultado de instabilidade orbital por perturbações subharmônicas.

Para o segundo modelo em (2), usando a onda solitária $\phi_{w, 0}$ no caso em que $Z=0$, obtemos duas famílias de picos solitários. Nós observamos que quando $Z \rightarrow 0$, temos que $\phi_{w, Z} \rightarrow \phi_{w, 0}$, onde $\phi_{w, 0}$ denota a onda solitária. Então, usando a teoria de perturbação analítica para operadores lineares não limitados, obtemos um resultado detalhado da estabilidade orbital de picos solitários.

Além disto, apresentamos alguns problemas naturais que podem ser resolvidos futuramente. Em particular, nós propomos uma nova abordagem para resolver questões de estabilidade linear de soluções de equilíbrio para certo tipo de equações parabólicas. 



\section{Abstract \\ Stability of travelling waves \\ for Schrödinger's equations \\ of cubic-quintic type}

This work is devoted to understand some mathematical aspects of the following nonlinear models: the nonlinear Schrödinger equation with double power in its non-linearity, that is

$$
i u_{t}+u_{x x}+u|u|^{2}+u|u|^{4}=0
$$

and a perturbation of delta type of this model, namely

$$
i u_{t}+u_{x x}+Z \delta(x) u+u|u|^{2}+u|u|^{4}=0 .
$$

For the first model, by using the theory of Jacobi elliptic integrals and the implicit function theorem, we obtain a family of standing waves $u(x, t)=e^{-i w t} \phi_{w}(x)$, where $\phi_{w}: \mathbb{R} \rightarrow \mathbb{R}$ is a positive periodic function of period $L>0$, known as the wave profile. When $L \rightarrow \infty$, we show that the periodic standing waves converge uniformly on compact intervals to the solitary waves. Moreover, using an extension of the Angulo\&Natali stability theory, as well as, the stability ideas developed by Weinstein, Bona, Grillakis, Shatah and Strauss, we show the orbital stability of the standing waves for perturbations of the same period of the wave profile. Finally, an orbital instability result by subharmonic perturbations is proved.

For the second model, by using the existence of the solitary wave $\phi_{w, 0}$ in the case $Z=0$, we obtain two families of solitary peaks. We observe that when $Z \rightarrow 0$, we have that $\phi_{w, Z} \rightarrow \phi_{w, 0}$, where $\phi_{w, 0}$ denotes the solitary wave. Then, using the analytic perturbation theory of unbounded linear operators, we obtain an accurate result about orbital stability of solitary peaks.

Furthermore, we give some natural problems that can be solved futurely. In particular, we propose a new approach to solve question of linear stability of equilibrium solutions for certain type of parabolic equations. 

1 Introdução 1

2 Notações e definições básicas $\quad 11$

$\begin{array}{lll}3 & \text { Teoria de estabilidade orbital } & 17\end{array}$

3.1 Estabilidade orbital . . . . . . . . . . . . . . . . . 17

3.2 Positividade da transformada de Fourier e estabilidade . . . . . . . . . 21

4 Existência e estabilidade orbital de ondas periódicas para a equação de Schrödinger cúbica-quíntica (CQ-S) 31

4.1 Boa colocação local e global para a equação CQ-S . . . . . . . . . . . . 32

4.2 Existência de ondas periódicas para a equação CQ-S . . . . . . . . . . . 41

4.3 Teoria espectral para a equação CQ-S . . . . . . . . . . . . . . . 49

4.4 Condição de convexidade da função $d \ldots \ldots$. . . . . . . . . . 55

4.5 Instabilidade por perturbações subharmônicas . . . . . . . . . . . 58

4.6 Estabilidade de soluções constantes . . . . . . . . . . . . . . 62

5 Equação de Schrödinger cúbica-quíntica com uma interação de tipo delta de Dirac (CQD-S)

5.1 Estabilidade de standing waves para a equação CQ-S no caso da reta . 66 
$5.2 \delta$-interação em dimensão um. . . . . . . . . . . . . . . . . . . 68

5.3 Boa colocação local e global para a equação CQD-S . . . . . . . . . . . 73

5.4 Existência de picos solitários para a equação CQD-S . . . . . . . . . . . 74

5.5 Estabilidade de standing waves para a equação CQD-S . . . . . . . . 78

5.5.1 Autovalores do operador $\mathcal{L}_{1, Z} \ldots \ldots \ldots$. . . . . . . . 81

5.5.2 Condição de convexidade . . . . . . . . . . . . . . . . . . . 89

5.5.3 Resultados de Estabilidade . . . . . . . . . . . . . . . . . . . 93

$\begin{array}{lll}6 & \text { Estudos Futuros } & 97\end{array}$

A Prova da estabilidade para a equação CQ-S 103

B Dinâmica da equação diferencial ordinária $a^{\prime \prime}+r a+s a^{3}+t a^{5}=0 \quad 109$ 


\section{CAPÍTULO 1}

Muitos dos complexos e interessantes fenômenos na natureza são devidos as interações não lineares de suas componentes. Devido à suas diversas aplicações, particularmente o chamado fenômeno de onda não linear (nonlinear wave phenomena) tem recebido bastante atenção nas últimas décadas dos pesquisadores das diferentes areas das ciências; Física: fluídos, ótica não linear, acústica e radio física; Matemática: equações diferenciais parciais, geometria diferencial, matemática computacional, análise numérica; engenheria, e.t.c.

As ondas em geral são um fenômeno comum em nossa vida diária. Mas, o que é uma onda não linear?. Um exemplo de ondas não lineares são aquelas que são vistas na mar. Dentro de águas profundas elas são pequenas, mas quando elas se movimentãm em direção da costa elas crescem e finalmente viram espuma. Outro exemplo, é o magnetron dentro de um micro-ondas. O magnetron usa campo magnético para gerar ondas eletrônicas, que usam uma interação não linear para produzir as micro-ondas que aquecem a comida. Além disso, temos certo tipo de onda não linear chamada soliton que foi proposta como portadoras de pedaços (bits) de informação em fibra óptica.

Mais exatamente, no estudo da física uma onda é uma perturbação que se propaga no espaço ou em qualquer outro meio, como, por exemplo, a água. Uma onda transfere 
energia de um ponto para outro, mas nunca transfere matéria entre dois pontos. Alguns dos fenômenos físicos que aparecem quando o fenômeno de onda é estudado são os seguintes: reflexão, refração polarização, difração, interferência, dispersão, e.t.c. Este último, é um fenômeno que acontece quando uma onda resultante da superposição de várias outras entra num meio onde a velocidade de propagação é diferente para cada uma de suas componentes. Conseqüentemente, a forma da função de onda inicial muda, sendo que sua forma é uma função do tempo. Por exemplo, a luz branca é formada por sete cores (vermelho, laranja, amarelo, verde, azul, azul escuro e violeta), que constituem seu espectro. Quando esta luz incide sobre um prisma de vidro, ela acaba sofrendo uma dispersão pois a velocidade da luz é diferente para cada cor e a luz branca acaba sofrendo uma decomposição nesta passagem. O violeta é o que sofre maior diminuição em sua velocidade ao passo que o vermelho é a cor que sofre a menor diminuição.

As ondas podem se classificar de acordo com a direção de propagação de energia, quanto à natureza das ondas e quanto à direção de propagação.

Quanto à direção de propagação de energia as ondas se classificam da seguinte forma:

1. Unidimensionais: propagam-se em uma única dimensão;

2. Bidimensionais: propagam-se num plano;

3. Tridimensionais: propagam-se em todas as direções.

Quanto à natureza, as ondas se classificam em:

1. Ondas mecânicas: são aquelas que necessitam de um meio material para se propagar como, por exemplo, onda em uma corda ou mesmo as ondas sonoras;

2. Ondas eletromagnéticas: são aquelas que não necessitam de meio material para se propagar, elas podem se propagar tanto no vácuo (ausência de matéria) como também em certos tipos de materiais. São exemplos de ondas eletromagnéticas: a luz solar, as ondas de rádio, as microondas, raios X, entre outras. 
Quanto à direção de propagação as ondas se classificam em:

1. Ondas transversais: são aquelas que têm a direção de propagação perpendicular à direção de vibração como, por exemplo, as ondas eletromagnéticas.

2. Ondas longitudinais: nessas ondas a direção de propagação se coincide com a direção de vibração. Nos líquidos e gases a onda se propaga dessa forma.

De particular importância, devido à suas diversas aplicações são as standing waves ou também conhecidas como stationary waves, este tipo de ondas é caracterizada pela sua não propagação. De fato este tipo de onda tem alguns pontos do meio fixados ou em posição constante durante a sua evolução. E pode surgir devido a que o meio se movimenta em direção oposta à onda ou também num meio estacionário como o resultado da interferência (interação) entre duas ondas viajando em direções opostas com a mesma amplitude e frequência. Para descrever melhor este tipo de onda consideremos a seguinte onda de tipo senoidal (ondas que tem um perfil parecido ao gráfico da função seno) $S_{+}(x)=\alpha \operatorname{sen}(k x-w t)$, aqui as variáveis $k, \alpha$ e $w>0$ denotam o número de onda, a amplitude e a frequência angular da onda, respectivamente. Já a variável $x$ denota o espaço e $t>0$ o tempo. Nestas condições, a função $S_{+}(x)$ representa então uma onda periódica viajando a uma velocidade $w$ na direção positiva do eixo $x$ tal que seu perfil é o mesmo para todo tempo $t$. Da mesma forma a função $S_{-}(x)=\alpha \operatorname{sen}(k x+w t)$ representa uma onda viajando a uma velocidade $w$ na direção negativa do eixo $x$. Como resultado da interação (soma) das duas ondas $S_{+}, S_{-}$, obtemos a standing wave $S=S_{+}+S_{-}$dada por $S(x, t)=\alpha \cos (w t) \operatorname{sen}(k x)$. Os pontos do espaço $x=0, \pm \frac{\pi}{k}, \pm \frac{2 \pi}{k}, \pm \frac{3 \pi}{k}, \cdots$, são os pontos estáticos da onda $S$ que surgem como a interação das ondas $S_{+}$e $S_{-}$. O resto de pontos do meio mudãm sua posição no tempo, embora de uma maneira muito repetida e regular. Descobrir este tipo de comportamento num médio físico é bastante simples comparado com outro tipo de comportamentos irregulares no meio.

Um dos aspectos fascinantes dentro do vasto mundo dos fenômenos não lineares (em particular ondas não lineares) são aqueles relacionados com a modelagem matemática 
de cada problema específico. Basicamente um modelo matemático é uma representação dos aspectos essenciais de um sistema existente (ou de um sistema a ser criado) que apresenta informação do sistema de uma maneira utilizável. Embora cada fenômeno não-linear tenha sua própria equação como modelo, é possível que exista uma estrutura não-linear comum entre as equações a qual pode permitir prever comportamentos similares das soluções. Por exemplo, no caso de equações de evolução dispersivas nãolineares (ou também lineares), muitos fenômenos no campo da física em particular no fenômeno de ondas não lineares podem ser modelados na forma abstrata (forma Hamiltoniana),

$$
u_{t}(x, t)=J E^{\prime}(u(x, t))
$$

onde $J$ é geralmente um operador anti-simétrico (skew-symmetric), $(x, t) \in \mathbb{R}^{n} \times \mathbb{R} \mathrm{e}$ $E^{\prime}$ é a derivada de Fréchet de um funcional geralmente chamado de energia ou primeira integral. Aqui $u=u(x, t)$ pode ser de valor real ou complexo ou ainda de valor vetorial.

Um exemplo clássico é a Equação de Schrödinger Não-Linear (NLS) (ver [20], [51])

$$
u_{t}=-i\left(-\Delta u-|u|^{p} u\right)
$$

onde $u=u(x, t) \in \mathbb{C}$ para $(x, t) \in \mathbb{R}^{n} \times \mathbb{R}$ e $p \in \mathbb{R}^{+}$. Comparando (1.2) com (1.1), neste caso temos que $J=-i$ e $E=E_{1}$ onde $E_{1}^{\prime}(u)=-\Delta u-|u|^{p}$ com

$$
E_{1}(u)=\int_{\mathbb{R}^{n}}\left[\frac{1}{2}|\nabla u|^{2}-\frac{1}{p+2}|u|^{p+2}\right] d x .
$$

Vários fenômenos físicos são governados pela equação (1.2) para $1 \leq n \leq 3$, a saber, na teoria não relativista da mecânica quântica, no estudo da propagação de ondas de laser, ondas langmuir em plasma, ondas de água, entre outros (ver [37]).

Outro importante exemplo é o da Equação de Korteweg-de Vries Generalizada $(\mathrm{GKdV})(\operatorname{ver}[32],[36])$

$$
u_{t}=\partial_{x}\left(-u_{x x}-u^{p}\right)
$$

onde $u=u(x, t) \in \mathbb{R}$ para $x, t \in \mathbb{R}, p \in \mathbb{N}, p \geqq 2$. Comparando (1.4) com (1.1), temos que $J=\partial_{x}$ e $E=E_{2}$ onde $E_{2}^{\prime}(u)=-u_{x x}-u^{p}$ com

$$
E_{2}(u)=\int_{\mathbb{R}}\left[\frac{1}{2}\left(u_{x}\right)^{2}-\frac{1}{p+1} u^{p+1}\right] d x .
$$


O caso $p=2$ em (1.4) é a conhecida equação de Korteweg-de Vries ou KdV, o caso $p=3$ é referida como a equação modificada de Korteweg-de Vries ou mKdV e o caso $p=5$ é a equação crítica de Korteweg-de Vries ou CKdV. Dependendo do valor de $p$, estes modelos surgem em vários contextos físicos, como por exemplo, para descrever a propagação de ondas de água de pequena amplitude e longitude de onda longa quando é comparada com a profundidade de um canal horizontal pouco profundo ou como modelo para ondas internas em um fluído estratificado $(p=2)$, ou na propagação de ondas pouco profundas em dimensão $2(p=3)$, ou em linhas de transmisão não-linear em eletrônica (ver Jeffrey-Kakutani [32] e Dodd et. al [24] para uma boa descrição dos contextos físicos relevantes onde a GKdV pode aparecer).

Do ponto de vista das equações diferenciais parciais dispersivas, alguns dos problemas que vem sendo abordados por muitos dos pesquisadores em procura de um melhor entendimento de ditos modelos e porém do fenômeno de onda não linear, são entre outros:

\section{Problemas de tipo local:}

Existência de soluções, unicidade, regularidade, efeito regularizante.

\section{Problemas de tipo global:}

Explosão em tempo finito (blow-up), existência global, comportamento assintótico das soluções, existência de ondas viajantes e a sua estabilidade (orbital/assintótica).

Particularmente, há algumas décadas atrás, o estudo da existência e estabilidade (orbital ou assintótica) de ondas viajantes do tipo onda solitárias vem possuindo um refinado e gratificante avanço. Muitas provas tem sido simplificadas e condições suficientes foram obtidas para assegurar a estabilidade/instabilidade destas ondas por pequenas perturbações localizadas (Albert [2], Albert\&Bona [1], Bona\&Souganidis\&Strauss [18], Grillakis\&Shatah\&Straus [27, 30], Weinstein $[48,49,50])$.

Comparavelmente, já o estudo da existência e estabilidade/instabilidade de ondas viajantes de tipo periódico tem recebido pouca atenção. Um primeiro estudo destas ondas foi iniciado por Benjamin em [14] com relação as ondas viajantes periódicas cha- 
madas de tipo cnoidal para a equação KdV (ver Eq. (1.4)). Em dito trabalho Benjamin não provou tal estabilidade de maneira completa e vários aspectos da sua prova parecem problemáticos. Recentemente, Angulo\&Bona\&Scialom em [9] conceberam uma teoria completa para a estabilidade destas ondas cnoidal pelo fluxo da KdV (ver também [8] e [7]). A abordagem para obter este resultado foi uma extensão da teoria moderna de estabilidade desenvolvida nos trabalhos de Weinstein\&Grillakis\&Shatah\&Strauss ([27], [48], [49]) ao caso periódico.

Podemos mencionar também que novas teorias de estabilidade orbital de ondas viajantes periódicas foram obtidas subsequentemente para outros modelos: equação de Schrödinger não linear (ver Eq. (1.2)) com $p=2,4$, em [4]-[6]-[10].

Agora, neste trabalho estamos interessados em entender algumas propriedades matemáticas dos seguintes modelos (para casos específicos das variaveis $\alpha, \beta, p, q$ )

\section{Equação de Schrödinger com dupla potência na não linearidade.}

$$
i u_{t}+u_{x x}+u\left(\alpha|u|^{p}+\beta|u|^{q}\right)=0
$$

$\operatorname{com} \alpha, \beta \in \mathbb{R}, 1<p<q<\infty$ e $u=u(x, t) \in \mathbb{C}$ para $(x, t) \in \mathbb{R} \times \mathbb{R}$.

A equação (1.6) aparece em vários contextos da física-matemática, por exemplo quando $\alpha>0, \beta<0, p=2, q=4$ aparece na interação gás-boson e ótica nãolinear (outras aplicações podem ser vistas em [12]). A estabilidade de soluções standing waves para a Eq. (1.6), a saber,

$$
u(x, t)=e^{-i w t} \phi(x)
$$

foi estudada de maneira completa e detalhada em Ohta [43] no espaço de energia $H^{1}(\mathbb{R})$ (ver definição 3.1.1 para o significado preciso de estabilidade).

\section{Equação de Schrödinger junto a uma interação de tipo delta de Dirac.}

$$
i u_{t}+u_{x x}+Z \delta(x) u+u\left(\alpha|u|^{p}+\beta|u|^{q}\right)=0, \quad Z \in \mathbb{R}
$$

onde $\delta$ é a medida de Dirac centrada no ponto $x=0, u: \mathbb{R} \times \mathbb{R} \rightarrow \mathbb{C}$, o par $(x, t)$ está definido sobre o conjunto $\mathbb{R} \times \mathbb{R}$ e $\alpha, \beta \in \mathbb{R}$. A interação entre a dispersão, 
a não linearidade, e o defeito (Delta de Dirac), produz uma ampla variedade de problemas matemáticos, os quais tem sido estudados por vários pesquisadores recentemente. Por exemplo Goodman, Holmes, Weinsten em [26] estudaram a estabilidade de standing waves para a equação (1.8) quando a não linearidade é cubica, isto é $p=2, \alpha=1, \beta=0$. Também Le Coz, et. al. em [38] fizeram um estudo analítico e numérico da estabilidade para a equação (1.8) quando $1<p<\infty, \alpha=1, \beta=0$. Do ponto de vista periódico Angulo\&Ponce em [11] fizeram um estudo da estabilidade de standing waves para a equação (1.8) quando $p=2, \alpha=1, \beta=0$.

Do ponto de vista matemático o estudo das soluções de tipo standing waves em (1.7) associadas aos modelos de tipo Schrödinger, como por exemplo os modelos (1.6) e (1.8), é interessante não só por ditas soluções serem soluções particulares de uma equação diferencial parcial não linear, mas também, porque um análise de uma ou algumas quantidades físicas associadas aos modelos como por exemplo a energia $\int \phi_{w}^{2}(x) d x$, podem determinar o comportamento qualitativo do fluxo da equação diferencial parcial perto da standing wave (ou mais precisamente, perto da órbita gerada pelo perfil $\phi_{w}$ em (1.7)). Este é informalmente a idéia da estabilidade orbital.

Na prática o entendimento do comportamento qualitativo das soluções para equações da forma (1.1) e especificamente dos modelos (1.6) e (1.8), é essencial para o desenvolvimento de vários projetos, por exemplo, na construção de instrumentos de tipo laser, na transmisão de dados em alta velocidade, transporte de ondas de matéria, propagação de raios óticos em meios não-standard, ou na recuperação de praias.

Desta maneira, o foco principal desta tese é estudar a estabilidade de tipo orbital (ver Def. 3.1.1) para os modelos (1.6) no caso periódico e (1.8) em toda a reta quando o valor dos parâmetros $\alpha, \beta, p$ e $q$ em (1.6)-(1.8) é dado por $\alpha=1, \beta=1, p=3$ e $q=5$. Assim para o modelo (1.6), mostramos a existência e estabilidade (ou instabilidade) de standing waves de tipo periódico para o modelo (1.8), encontramos uma família de standing waves de tipo picos e dependendo do sinal do parâmetro $Z$ determinamos a sua estabilidade ou instabilidade não linear. 
Mais especificamente, no caso do modelo (1.6), nós encontramos uma família de standing waves do tipo (1.7) com $\phi_{w}: \mathbb{R} \rightarrow \mathbb{R}$, periódica de periódo $L>0$, onde o perfil $\phi_{w}$ satisfaz a equação

$$
\phi_{w}^{\prime \prime}+w \phi_{w}+\phi_{w}^{3}+\phi_{w}^{5}=0
$$

Usando a teoria das funções elípticas de Jacobi, encontramos um perfil periódico da forma

$$
\phi_{w}(\xi)=\sqrt{\alpha_{3}} \frac{d n\left(\frac{2 \xi}{\sqrt{3} g} ; k\right)}{\sqrt{1+\beta^{2} \operatorname{sn}^{2}\left(\frac{2 \xi}{\sqrt{3} g} ; k\right)}},
$$

com um período minimal $L$, onde $d n$ e $s n$ representam as funções dnoidal e snoidal respectivamente (ver seção 4.2). Quando $L \rightarrow \infty$ temos que o perfil $\phi_{w}$ tende uniformemente sobre intervalos compactos à onda solitária associada a equação (1.9), à saber, para $w<0$,

$$
\phi_{s}(\xi)=\left[\frac{1}{-4 w}+\frac{\sqrt{9-48 w}}{-12 w} \cosh (2 \sqrt{-w} \xi)\right]^{-\frac{1}{2}} .
$$

Assim, estendendo a teoria de Angulo\&Natali em [5] para o estudo da estabilidade de ondas viajantes de tipo periódico, mostramos que a órbita gerada pelo perfil (1.10), a saber,

$$
\Omega_{\phi_{w}}=\left\{e^{i \theta} \phi_{w}(\cdot+y) \mid \theta \in[0,2 \pi), y \in \mathbb{R}\right\},
$$

resulta ser estável em $H_{\text {per }}^{1}([0, L])$ pelo fluxo periódico da equação (1.6) (ver capítulo 3 para a definição formal de estabilidade orbital). Neste ponto o estudo dos operadores auto-adjuntos de tipo Hill,

$$
\begin{aligned}
\mathcal{L}_{R} & =-\frac{d^{2}}{d x^{2}}-w-3 \phi_{w}^{2}-5 \phi_{w}^{4}, \\
\mathcal{L}_{\text {Im }} & =-\frac{d^{2}}{d x^{2}}-w-\phi_{w}^{2}-\phi_{w}^{4},
\end{aligned}
$$

é parte fundamental para nossa teoria de estabilidade.

Além disso, provamos que as soluções em (1.7) com perfil (1.10) resultam ser instáveis por perturbações de tipo subharmônico, ou seja, perturbações no espaço $H_{p e r}^{1}([0, j L])$, com $j \geq 2$. 
Agora, para o modelo (1.8), baseados no perfil $\phi_{s}$ em (1.11) nós mostramos a existência de uma familia de picos solitários. Esta família vem dada explicitamente por

$$
\phi_{w, Z}(x)=\left[-\frac{1}{4 w}-\frac{\sqrt{9-48 w}}{12 w} \cosh \left(2 \sqrt{-w}\left(|x|+R^{-1}\left(\frac{Z}{2 \sqrt{-w}}\right)\right)\right)\right]^{-\frac{1}{2}}
$$

com

$$
R(b)=\frac{\beta \sinh (2 \sqrt{-w} b)}{\alpha+\beta \cosh (2 \sqrt{-w} b)},
$$

sendo um difeomorfismo crescente de $\mathbb{R}$, no intervalo $(-1,1)$ e os parâmetros $Z, w$ satisfazendo a relação

$$
\frac{Z^{2}}{4}<-w
$$

Notamos que para $Z \rightarrow 0$ temos que $\phi_{w, Z} \rightarrow \phi_{s}$ em $H^{1}(\mathbb{R})$. Assim, usando a teoria analítica de perturbação para operadores lineares, nos mostramos que a órbita gerada por $\phi_{w, Z}$, a saber,

$$
\Omega_{\phi_{w, Z}}=\left\{e^{i \theta} \phi_{w, Z} \mid \theta \in[0,2 \pi)\right\}
$$

resulta ser estável em $H^{1}(\mathbb{R})$ para $Z>0$ e instável em $H^{1}(\mathbb{R})$ para $Z \in\left(Z^{*}, 0\right)$, onde $Z^{*}$ é um valor negativo determinado.

Observamos que a órbita estudada em (1.15) é diferente de aquela em (1.12), pois o modelo em (1.6) resulta ser invariante por rotações e translações, já o modelo (1.8), resulta ser apenas invariante por rotações por causa da presença da função delta. Neste ponto também o estudo do espectro dos operadores lineares auto-adjuntos $\mathcal{L}_{i, Z}: \mathcal{D} \rightarrow$ $L^{2}(\mathbb{R}), i=1,2$, dados por

$$
\begin{aligned}
& \mathcal{L}_{1, Z} g=-\frac{d^{2}}{d x^{2}} g-w g-3 \phi_{w, Z}^{2} g-5 \phi_{w, Z}^{4} g, \\
& \mathcal{L}_{2, Z} g=-\frac{d^{2}}{d x^{2}} g-w g-\phi_{w, Z}^{2} g-\phi_{w, Z}^{4} g .
\end{aligned}
$$

com domínio

$$
\mathcal{D} \equiv D\left(\mathcal{L}_{i, Z}\right)=\left\{g \in H^{1}(\mathbb{R}) \cap H^{2}(\mathbb{R}-\{0\}) \mid g^{\prime}(0+)-g^{\prime}(0-)=-Z g(0)\right\},
$$

é vital para as nossas conclusões. 
Para compreender melhor os resultados obtidos, nosso trabalho será dividido nos seguintes capítulos:

No Capítulo 2, apresentamos as notações e definições básicas que serão utilizadas em nosso trabalho. No capítulo 3 estabeleceremos as idéias básicas de nossa teoria geral de estabilidade que sera usada em nosso trabalho. Em particular damos uma extensão da teoria de Angulo\&Natali em [5]. No capítulo 4 estabelecemos a existência e estabilidade de ondas viajantes periódicas para a equação CQ-S $(\alpha=1, \beta=1, p=3$ e $q=5$ no modelo 1.6). Em particular mostramos uma teoria de boa-colocação local e global para o problema de Cauchy associado a Eq. CQ-S. Também uma teoria de instabilidade por perturbações subharmônicas e estabilidade de standing waves com perfil constante, é exibida. No Capítulo 5 apresentamos a teoria de estabilidade e instabilidade em $H^{1}(\mathbb{R})$ das soluções picos dadas em (1.14). Istos resultados serão baseados na teoria de perturbação analítica para operadores lineares não limitados. Para um melhor entendimento de nosso estudo apresentamos neste capítulo os principais resultados associados ao operador

$$
-\frac{d^{2}}{d x^{2}}+\gamma \delta
$$

em dimensão um. O uso da teoria de Von Neumann será fundamental neste ponto. Também mostramos uma teoria de boa colocação local e global para o problema de Cauchy associado a Eq. CQD-S $(\alpha=1, \beta=1, p=3$ e $q=5$ no modelo (1.8)). No capítulo 6 apresentamos alguns problemas que poderiam ser abordados em um estudo futuro. No apêndice A, estabelecemos uma prova geral do teorema de estabilidade associado ao modelo CQ-S. Notamos que esta prova também funciona no caso da Eq. CQD-S. Finalmente, no apêndice B, fazemos um estudo da dinâmica no plano de fase associada a equação diferencial $a^{\prime \prime}+r a+s a^{3}+t a^{5}=0$. Este estudo permite entender melhor as possíveis soluções periódicas e não periódicas que poderiam ser estudadas num futuro breve. 


\section{CAPÍTULO 2}

\section{NOTAÇÕES E DEFINIÇÕES BÁSICAS}

Neste capítulo, apresentamos algumas notações, definições e resultados básicos que serão usados ao longo deste trabalho.

1- Os símbolos $\mathbb{N}, \mathbb{Z}, \mathbb{R}$ e $\mathbb{C}$, denotam o conjunto dos números naturais, inteiros, reais e complexos, respectivamente.

2- A norma de uma função $f \in L^{p}(\Omega)$, para $\Omega$ um subconjunto aberto de $\mathbb{R}$, será denotada por $\|f\|_{L^{p}}^{p}=\int_{\Omega}|f(x)|^{p} d x, p \geq 1$.

3- O produto interno em $L^{2}(\Omega)$ de duas funções $f, g$ é denotado por $(f, g)=\int_{\Omega} f(x) \overline{g(x)} d x$.

4- $B(X, Y)$, denota o conjunto dos operadores limitados do espaço de Banach $X$ no espaço de Banach $Y$.

5- Para definir os espaços de Sobolev (de tipo $L^{2}$ ) de funções periódicas, usamos os conceitos e as notações em Iório [31]. Assim, seja $P$ o espaço de Fréchet dado por

$$
P:=C_{\text {per }}^{\infty}([-l, l]):=\left\{f: \mathbb{R} \rightarrow \mathbb{C} \mid f \in C^{\infty}(\mathbb{R}) \text { é periódica com período } 2 l>0\right\} .
$$

munido com à topologia gerada pelas seminormas $\|\cdot\|_{k}$, definidas sobre $P$ por

$$
\|h\|_{k}=\sup _{x \in[-l, l]}\left|h^{(k)}(x)\right| .
$$

O conjunto das distribuições periódicas ou também chamado de dual topológico do espaço $P$, é denotado por $P^{*}$ e se define como a coleção de todos os funcionais lineares 
contínuos de $P$ em $\mathbb{C}$. Se $\psi \in P^{*}$ denotamos o valor de $\psi$ em $\varphi$ por $\psi(\varphi)=\langle\psi, \varphi\rangle$. Definindo as funções $\Theta_{k}$, por

$$
\Theta_{k}(x):=e^{i k \pi x / l}
$$

para $k \in \mathbb{Z}$ e $x \in \mathbb{R}$. Então a transformada de Fourier do funcional $\psi \in P^{*}$ é definida como a função $\widehat{\psi}: \mathbb{Z} \rightarrow \mathbb{C}$ dada pela fórmula

$$
\widehat{\psi}(k)=\frac{1}{2 l}\left\langle\psi, \Theta_{-k}\right\rangle, k \in \mathbb{Z} .
$$

Assim, se $\psi$ é uma função periódica com periodo $2 l$, por exemplo $\psi \in L^{2}(-l, l)$, e definimos $\psi_{0} \in P^{*}$ por $\psi_{0}(h)=\frac{1}{2 l} \int_{-l}^{l} \psi(x) h(x) d x$, temos então que

$$
\widehat{\psi_{0}}(k)=\frac{1}{2 l} \int_{-l}^{l} \psi(x) e^{-i k \pi x / l} d x,
$$

que é a transformada de Fourier canônica no espaço $L^{2}(-l, l)$. Seja $s \in \mathbb{R}$, o espaço de Sobolev $H_{p e r}^{s}([-l, l])$ é o conjunto de todos os $f \in P^{*}$ tal que

$$
\|f\|_{s}^{2}=2 l \sum_{k=-\infty}^{\infty}\left(1+|k|^{2}\right)^{s}|\widehat{f}(k)|^{2}<\infty
$$

$H_{p e r}^{s}([-l, l])$ é um espaço de Hilbert com respeito ao produto interno

$$
(f \mid g)_{s}=2 l \sum_{k=-\infty}^{\infty}\left(1+|k|^{2}\right)^{s} \widehat{f}(k) \overline{\widehat{g}(k)} .
$$

No caso $s=0$, obtemos um espaço de Hilbert que é isometricamente isomorfo a $L^{2}(-l, l), \operatorname{logo}$ temos $(f \mid g)_{0}=(f, g)=\int_{-l}^{l} f \bar{g} d x . \quad H_{p e r}^{0}([-l, l])$ sera denotado por $L_{\text {per }}^{2}([-l, l]) \operatorname{com}\|\cdot\|_{0}=\|\cdot\|$. Como $H_{\text {per }}^{s}([-l, l]) \subset L_{\text {per }}^{2}([-l, l])$ para todo $s \geq 0$, temos então pela identidade de Parseval que para todo $n \in \mathbb{N}$

$$
\|f\|_{n}^{2}=\sum_{j=0}^{n}\left\|f^{(j)}\right\|^{2}=\sum_{j=0}^{n} \int_{-l}^{l}\left|f^{(j)}(x)\right|^{2} d x,
$$

onde $f^{(j)}$ representa a derivada de ordem $j$ da função $f$ tomada no sentido de $P^{*}$. Além disso, $\left(H_{p e r}^{s}([-l, l])\right)^{*}$, o dual topológico de $H_{p e r}^{s}([-l, l])$, é isometricamente isomorfo a $H_{p e r}^{-s}([-l, l])$ para todo $s \in \mathbb{R}$. A dualidade é dada pelo parênteses

$$
\langle f, g\rangle_{s}=2 l \sum_{k=-\infty}^{\infty} \widehat{f}(k) \bar{g}(k), f \in H_{p e r}^{-s}([-l, l]), g \in H_{p e r}^{s}([-l, l]) .
$$


Então, se $f, g \in L_{p e r}^{2}([-l, l])$ segue que $\langle f, g\rangle_{s}=(f, g)$.

6- $S(\mathbb{Z}):=\left\{\alpha: \mathbb{Z} \rightarrow \mathbb{C}\left|\forall n \in \mathbb{N}\|\alpha\|_{n}=\sup _{k \in \mathbb{Z}}\right| k^{n} \alpha_{k} \mid<\infty\right\}$.

7- Para $T>0, C([0, T] ; X)$ denota o espaço de Banach das funções contínuas do intervalo $[0, T]$ no espaço de Banach $X$. Aqui a norma no espaço $C([0, T] ; X)$ é dada por

$$
\|u\|=\sup _{x \in[0, T]}\|u(t)\|_{X}
$$

8- Para $s \in \mathbb{R}$, os espaços de Sobolev $H^{s}(\mathbb{R})$ são definidos por

$$
H^{s}(\mathbb{R})=\left\{\left.f \in S^{*}(\mathbb{R})\left|\int_{-\infty}^{\infty}\left(1+\xi^{2}\right)^{s}\right| \widehat{f}(\xi)\right|^{2} d \xi<\infty\right\}
$$

com $S^{*}(\mathbb{R})$, o espaço das distribuições temperadas, o dual topológico do espaço de Schwartz,

$$
S(\mathbb{R})=\left\{f \in C^{\infty}(\mathbb{R})\left|\|f\|_{k, m}=\sup _{x \in \mathbb{R}}\right| x^{k} f^{(m)}(x) \mid<\infty \forall k, m \in \mathbb{N}\right\}
$$

Aqui, as seminormas $\|\cdot\|_{k, m}$ definidas no espaço $S(\mathbb{R})$ geram uma topologia que o tornam um espaço de Frechet. Na definição (2.1) a transformada de Fourier $\widehat{f}\left(\in S^{*}(\mathbb{R})\right)$ de uma função $f \in S^{*}(\mathbb{R})$, é definida por $\langle\widehat{f}, g\rangle:=\langle f, \widehat{g}\rangle \operatorname{com} g \in S(\mathbb{R})$ e

$$
\widehat{g}(\xi)=\frac{1}{2 \pi} \int_{-\infty}^{\infty} e^{-i x \xi} f(x) d x
$$

9- Para $\Omega$ um conjunto aberto de $\mathbb{R}, m \in \mathbb{N}, H^{m}(\Omega)$ denota o espaço de Hilbert de funções mensuraveis $u: \Omega \rightarrow \mathbb{C}$ tal que $u^{(i)} \in L^{2}(\Omega)$ no sentido de distribuições, para $i \in \mathbb{N}$, com $0 \leq i \leq m$. Aqui o produto interno no espaço $H^{m}(\Omega)$ é dada por

$$
(u, v)_{m}=\sum_{0 \leq i \leq m} \int_{\Omega} u^{(i)}(x) \overline{v^{(i)}(x)} d x .
$$

Agora, apresentamos as definições das funções elípticas de Jacobi (ver [15]). Em nosso trabalho, tais funções são fundamentais na análise das soluções standing waves periódicas associadas ao problema de estabilidade orbital do modelo (1.6). Em particular, elas nos permitirão calcular explicitamente ditas soluções, assim como analizar 
seu período, a sua energia, e.t.c (ver capítulo 4). Desta forma temos:

1- Integral elíptica normal de primeiro tipo,

$$
\begin{aligned}
\int_{0}^{y} \frac{d t}{\sqrt{\left(1-t^{2}\right)\left(1-k^{2} t^{2}\right)}} & =\int_{0}^{\varphi} \frac{d \vartheta}{\sqrt{1-k^{2} \operatorname{sen}^{2}(\vartheta)}} \\
& \int_{0}^{u_{1}} d u=u_{1} \equiv \operatorname{sn}^{-1}(y, k) \equiv F(\varphi, k),
\end{aligned}
$$

$\operatorname{com} y=\operatorname{sen}(\varphi)$ e $\varphi=a m u_{1}$.

\section{2- Integral elíptica normal de segundo tipo,}

$$
\begin{aligned}
\int_{0}^{y} \sqrt{\frac{1-k^{2} t^{2}}{1-t^{2}}} d t & =\int_{0}^{\varphi} \sqrt{1-k^{2} \operatorname{sen}^{2}(\vartheta)} d \vartheta=\int_{0}^{u_{1}} d n^{2}(u) d u \\
& =E\left(u_{1}\right) \equiv E\left(\operatorname{am~} u_{1}, k\right) \equiv E(\varphi, k) .
\end{aligned}
$$

A segunda forma das integrais que aparece nas fórmulas (2.2)-(2.3), é a notação dada por Legendre. Já a primeira e terceira forma são notações devidas a Jacobi. O número $k$ é chamado o módulo da integral e pode ter valores reais ou complexos, mas com algumas transformações ele pode ser considerado satisfazendo $0<k<1$.

O número $k^{\prime}:=\sqrt{1-k^{2}}$, é conhecido como o módulo complementar da integral. As variáveis $y$ ou $\varphi$, denotam o chamado argumento, usualmente satisfazendo $0<y \leq 1$ ou $0<\varphi \leq \frac{\pi}{2}$.

3- Integrais elípticas completas, estas são obtidas quando $y=1$ em (2.2), (2.3), da seguinte maneira

$$
\int_{0}^{\frac{\pi}{2}} \frac{d \vartheta}{\sqrt{1-k^{2} \operatorname{sen}^{2}(\vartheta)}}=\int_{0}^{K} d u=F\left(\frac{\pi}{2}, k\right) \equiv K(k) \equiv K,
$$

e

$$
\int_{0}^{\frac{\pi}{2}} \sqrt{1-k^{2} \operatorname{sen}^{2}(\vartheta)} d \vartheta=\int_{0}^{K} d n^{2}(u) d u=E\left(\frac{\pi}{2}, k\right) \equiv E(k) \equiv E .
$$

4- Também definimos as integrais completas $K^{\prime}, E^{\prime}$, por

$$
\begin{aligned}
& K^{\prime}(k) \equiv K\left(k^{\prime}\right) \equiv K^{\prime} \\
& E^{\prime}(k) \equiv E\left(k^{\prime}\right) \equiv E^{\prime} .
\end{aligned}
$$


5- A função Lambda de Heuman, é definida por

$$
\Lambda_{0}(\beta, k) \equiv \frac{2}{\pi}\left[E(k) F\left(\beta, k^{\prime}\right)+K(k) E\left(\beta, k^{\prime}\right)-K(k) F\left(\beta, k^{\prime}\right)\right] .
$$

6- Escrevendo $u\left(y_{1}, k\right) \equiv u=\int_{0}^{y_{1}} \frac{d t}{\sqrt{\left(1-t^{2}\right)\left(1-k^{2} t^{2}\right)}}($ ver $(2.2))$, é possivel então definir as funções inversas

$$
y_{1}=\operatorname{sn}(u, k)
$$

e

$$
\varphi=\operatorname{am}(u, k)
$$

ou brevemente $y_{1}=s n(u), \varphi=a m u$ quando não é necessario enfatizar o modulo.

Também definimos as funções

$$
c n(u, k)=\sqrt{1-y_{1}^{2}}=\cos (\varphi)
$$

e

$$
d n(u, k)=\sqrt{1-k^{2} y_{1}^{2}}=\sqrt{1-k^{2} \operatorname{sen}^{2}(\varphi)} .
$$

As funções $s n(u), c n(u)$ e $d n(u)$ são chamadas funções elípticas de Jacobi do tipo snoidal, cnoidal e dnoidal, respectivamente. Essas funções possuem períodos reais iguais á $4 K, 4 K$ e $2 K$, respectivamente. Alem disso, temos as seguintes identidades básicas $s n^{2}(u)+c n^{2}(u)=1, \operatorname{sn}(u, 0)=\operatorname{sen}(u), \operatorname{cn}(u, 0)=\cos (u), \operatorname{sn}(u, 1)=\tanh (u)$ e $\operatorname{cn}(u, 1)=\operatorname{sech}(u)$.

7- Função Zeta de Jacobi $Z$, é definida por

$$
\begin{aligned}
Z\left(u_{1}\right) \equiv \int_{0}^{u_{1}}\left[d n^{2}(u)-\frac{E}{K}\right] d u & =E\left(u_{1}\right)-\frac{E}{K} u_{1} \\
& =E(\beta, k)-\frac{E}{K} F(\beta, k),
\end{aligned}
$$

onde $\beta=a m u_{1}$.

8- Função Theta $\Theta$, é definida pela série de Fourier

$$
\Theta(u)=\vartheta_{0}(v)=1+2 \sum_{m=1}^{\infty}(-1)^{m} q^{\left(m^{2}\right)} \cos (2 m v),
$$


$\operatorname{com} q=e^{-\pi \frac{K^{\prime}}{K}}$ e $v=\frac{\pi u}{2 K}$.

9- As seguintes fórmulas serão importantes no cálculo da convexidade de uma função básica no estudo da estabilidade (ver seção 4.4)

$$
\begin{aligned}
& \frac{d K}{d k}=\frac{E(k)-\left(1-k^{2}\right) K(k)}{k\left(1-k^{2}\right)}, \\
& \frac{d}{d \varphi} \Lambda_{0}(\varphi, k)=\frac{2\left(E(k)-\left(1-k^{2}\right) \sin ^{2}(\varphi) K(k)\right)}{\pi \sqrt{1-\left(1-k^{2}\right) \sin ^{2}(\varphi)}}, \\
& \frac{d}{d k} \Lambda_{0}(\varphi, k)=\frac{2(E(k)-K(k)) \sin (\varphi) \cos (\varphi)}{\pi k \sqrt{1-\left(1-k^{2}\right) \sin ^{2}(\varphi)}}, \\
& \frac{d}{d \varphi} F(\varphi, k)=\frac{1}{\sqrt{1-k^{2} \sin ^{2}(\varphi)}}, \\
& \frac{d}{d k} F(\varphi, k)=\frac{E(\varphi, k)-\left(1-k^{2}\right) F(\varphi, k)}{k\left(1-k^{2}\right)}-\frac{k \sin (\varphi) \cos (\varphi)}{\left(1-k^{2}\right) \sqrt{1-k^{2} \sin ^{2}(\varphi)}}
\end{aligned}
$$

10- Se $f: \mathbb{R} \rightarrow \mathbb{R}$, diremos que $f$ está na classe $P F(2)$ contínuo se

i) $f(x)>0$, para todo $x \in \mathbb{R}$.

ii) $f\left(x_{1}-y_{1}\right) f\left(x_{2}-y_{2}\right)-f\left(x_{1}-y_{2}\right) f\left(x_{2}-y_{1}\right)>0$, para $x_{1}<x_{2}$ e $y_{1}<y_{2}$.

O seguinte lema foi estabelecido por Albert e Bona em [1] e dá uma condição suficiente para mostrar quando uma função suave e positiva está na classe $P F(2)$ contínuo.

Lema 2.0.1. Suponha que $f$ é uma função positiva, duas vezes diferenciável sobre $\mathbb{R}$ e satisfazendo $\frac{d^{2}}{d x^{2}} \log (f(x))<0$, para todo $x \neq 0$. Então $f \in P F(2)$ contínuo.

Para mais detalhes sobre a propriedade $P F(2)$ contínuo, ver capítulo 7 de Karlin [34]. 


\section{CAPÍTULO 3}

\section{TEORIA DE ESTABILIDADE ORBITAL}

Neste capítulo apresentaremos as idéias gerais da teoria desenvolvida em [27]. Esta será usada nos capítulos posteriores para mostrar a estabilidade orbital de ondas viajantes periódicas para diferentes modelos de tipo dispersivo.

Também faremos uma extensão da teoria desenvolvida em [5]. Esta nos permitirá estudar as propriedades espectrais de operadores lineares auto-adjuntos associados ao problema de estabilidade ou instabilidade de ondas viajantes periódicas para modelos gerais do tipo apresentado em (3.20) abaixo.

\subsection{Estabilidade orbital}

Nesta seção vamos deixar claro os aspectos essenciais que devem ser estudados para obter uma teoria de estabilidade orbital de ondas viajantes para uma equação de evolução de tipo dispersivo. As idéias aqui expostas fazem parte da teoria desenvolvida por Grillakis\&Shatah\&Strauss em [27], Benjamin em [13]-[14], Bona [16] e Weinstein [48], ao caso periódico. Nosso intuito é deixar claro os pontos principais que aparecem em tais teorias, assim que muitas das nossas afirmações nesta seção serão justificadas ou entendidas melhor nos próximos capítulos ou mesmo nos apêndices finais.

Consideramos a equação de Schrödinger Cúbica-Quíntica (CQ-S), definida da se- 
guinte forma

$$
\begin{aligned}
& i u_{t}+u_{x x}+u\left(|u|^{2}+|u|^{4}\right)=0, \quad(x, t) \in \mathbb{R} \times \mathbb{R}, \\
& u(0)=u_{0} \in X=H_{p e r}^{1}([0, L]),
\end{aligned}
$$

com $u: \mathbb{R} \times \mathbb{R} \rightarrow \mathbb{C}$ para $(x, t) \in \mathbb{R} \times \mathbb{R}$ e $L>0$ representa um período fixado. Sejam $T_{1}, T_{2}$ grupos unitários a um parâmetro definidos sobre $L^{2}([0, L])$, como,

$$
\begin{aligned}
& T_{1}(\theta) \phi=e^{-\theta i} \phi, \quad \text { para } \phi \in L^{2}([0, L]), \theta \in \mathbb{R}, \\
& T_{2}(s) \phi(\cdot)=\phi(\cdot-s), \quad \text { para } \phi \in L^{2}([0, L]), s \in \mathbb{R} .
\end{aligned}
$$

Os geradores infinitesimais de $T_{1}$ e $T_{2}$ são dados por $T_{1}^{\prime}(0)=-i$ e $T_{2}^{\prime}(0)=\frac{d}{d x}$ respectivamente. Notamos que a equação (3.1a) tem as simetrias de rotação e translação, isto significa que se $u=u(x, t)$ é uma solução da equação CQ-S então $v_{\theta}(x, t):=$ $T_{1}(\theta) u(\cdot, t)=e^{-\theta i} u(x, t)$ e $v_{s}(x, t):=T_{2}(s) u(\cdot, t)=u(x-s, t)$ continuam sendo soluções de (3.1a).

Vamos fazer as seguintes hipóteses:

H-0 O problema de Cauchy (3.1) é globalmente bem posto.

H-1 As soluções particulares do tipo standing waves para (3.1a) que serão estudadas tem a forma

$$
u(x, t)=T_{1}(w t) a(x)
$$

onde $w \in \mathbb{R}$ e $a: \mathbb{R} \rightarrow \mathbb{R}$ é uma de função periódica com período fixado $L$. Aqui o perfil da standing wave $a$ precisa satisfazer a seguinte equação diferencial ordinária não-linear

$$
a^{\prime \prime}(x)+w a(x)+a^{3}(x)+a^{5}(x)=0 \quad \forall x \in \mathbb{R} .
$$

Vamos supor que existe uma curva suave $w \in \Omega \subset \mathbb{R} \rightarrow a_{w} \in X$ de ondas periódicas com um período fixado $L>0$, aqui $\Omega$ denota um intervalo aberto.

H-2 Cada solução $a_{w}$ é um ponto crítico do funcional $\Psi: X \rightarrow \mathbb{R}$, definido como

$$
\Psi=E-w F
$$


onde $E, F: X \rightarrow \mathbb{R}$ são os seguintes funcionais suaves,

$$
\begin{aligned}
& E(u)=\frac{1}{2} \int_{0}^{L}\left|u_{x}\right|^{2}-\frac{|u|^{4}}{2}-\frac{|u|^{6}}{3} d x \\
& F(u)=\frac{1}{2} \int_{0}^{L}|u|^{2} d x .
\end{aligned}
$$

Notamos que $E, F$ são quantidades conservadas pelo fluxo da CQ-S e invariantes pelos grupos $T_{1}, T_{2}$. A saber para qualquer $\theta, s \in \mathbb{R}, u \in X$,

$$
\begin{aligned}
& E\left(T_{k}(s) u\right)=E(u), \\
& F\left(T_{k}(s) u\right)=F(u),
\end{aligned}
$$

$k=1,2$, e para $u(t)$ sendo o fluxo da equação (3.1a), temos formalmente para qualquer $t \in \mathbb{R}$ que

$$
\begin{aligned}
& E(u(t))=E(u(0)), \\
& F(u(t))=F(u(0)) .
\end{aligned}
$$

Agora introduzimos a nossa definição de estabilidade orbital para soluções da forma (3.3) associadas à CQ-S,

Definição 3.1.1. (Estabilidade Orbital para a $C Q-S$ ). Consideramos a órbita gerada por $a_{w}$

$$
\Omega_{a_{w}}=\left\{T_{1}(\theta) T_{2}(s) a_{w}:(s, \theta) \in \mathbb{R} \times \mathbb{R}\right\}
$$

e para $\eta>0$ definimos $U_{\eta} \subset X$ por

$$
f \in U_{\eta} \Leftrightarrow \rho\left(f, \Omega_{a_{w}}\right)=\inf _{g \in \Omega_{a_{w}}}\|f-g\|_{X}<\eta .
$$

Dizemos então que $\Omega_{a_{w}}$ é estável em X pelo fluxo periódico gerado por Eq.(3.1a), se para todo $\epsilon>0$, existe $\delta(\epsilon)>0$ tal que para todo $u_{0} \in U_{\delta}$, a solução u do problema de valor inicial (3.1a)-(3.1b) satisfaz $u(t) \in U_{\epsilon}$ para todo $t \in \mathbb{R}$. Caso contrário, $\Omega_{a_{w}}$ é chamada instável em $X$.

Agora, usando H-2 e o teorema de Taylor é possível obter a seguinte aproximação do funcional $\Psi$ ao redor de $a_{w} \in X$

$$
\Psi(u)-\Psi\left(a_{w}\right)=\left\langle\Psi^{\prime \prime}\left(a_{w}\right)\left(u-a_{w}\right), u-a_{w}\right\rangle+o\left(\left\|u-a_{w}\right\|^{2}\right)
$$


onde $\Psi^{\prime \prime}\left(a_{w}\right)$ representa a segunda variação ou Hessiano do funcional $\Psi$ em $a_{w}$. Assim não é dificil ver que para $\phi=z_{1}+i z_{2}$, a forma quadrática associada ao Hessiano $\Psi^{\prime \prime}\left(a_{w}\right)$ é dada por

$$
\left\langle\Psi^{\prime \prime}\left(a_{w}\right) \phi, \phi\right\rangle=\left\langle\mathcal{L}_{R} z_{1}, z_{1}\right\rangle+\left\langle\mathcal{L}_{\text {Im }} z_{2}, z_{2}\right\rangle
$$

onde os operadores não-limitados e auto-adjuntos $\mathcal{L}_{R}$ e $\mathcal{L}_{I m}$ estão definidos sobre $H_{p e r}^{2}([0, L])$ como

$$
\begin{aligned}
\mathcal{L}_{R} & =-\frac{d^{2}}{d x^{2}}-w-3 a^{2}-5 a^{4}, \\
\mathcal{L}_{\text {Im }} & =-\frac{d^{2}}{d x^{2}}-w-a^{2}-a^{4},
\end{aligned}
$$

para $w \in \Omega$ e $a=a_{w}$ satisfazendo (3.4). O objetivo à seguir é assegurar que o operador $\Psi^{\prime \prime}\left(a_{w}\right)$ é de certa forma coercivo quando restringimos a alguma variedade específica em $H_{p e r}^{1}([0, L])$. Para isso, precisamos de algumas hipóteses sobre o comportamento dos operadores $\mathcal{L}_{R}$ e $\mathcal{L}_{\text {Im }}$ definidos em (3.12). Assim, temos as seguintes condições sobre o espectro associado ao operador $\mathcal{L}_{R}$ :

H-3.1 Existe um único autovalor negativo e ele é simples.

H-3.2 Zero é autovalor e é simples.

H-3.3 O resto do espectro é positivo e está distante de zero.

Sobre o espectro do operador $\mathcal{L}_{I m}$ temos o seguinte:

H-4.1 Zero é o primeiro autovalor e é simples.

H-4.2 O resto do espectro é positivo e está distante de zero.

A seguir definimos a função escalar $d: \Omega \rightarrow \mathbb{R}$, como

$$
d(w):=E\left(a_{w}\right)-w F\left(a_{w}\right)
$$

Esta função como veremos vai determinar de forma precisa a dinâmica da órbita $\Omega_{a_{w}}$. Agora, definimos a seguinte variedade para $a=a_{w}, w$ fixado.

$$
P=\left\{[p, q] \in X \times X \mid\langle a, p\rangle=0,\left\langle a^{\prime}, p\right\rangle=0,\langle a, q\rangle=0\right\}
$$


Então combinando as hipóteses H-1, H-2, H-3, H-4, mostraremos no apêndice A o seguinte resultado de coercividade para o operador $\Psi^{\prime \prime}(a)$.

Lema 3.1.1. Se $d^{\prime \prime}(w)>0$, então existe uma constante $\delta>0$ tal que para qualquer $[p, q] \in P$, definido em (3.14), temos

$$
\left\langle\Psi^{\prime \prime}\left(a_{w}\right)[p, q],[p, q]\right\rangle \geq \delta\|[p, q]\|_{H^{1}}^{2}
$$

Assim, seguindo as idéias do Teorema 3.4 em [27], podem-se obter constantes $c_{1}>$ $0, \delta_{1}>0$, tal que,

$$
E(u)-E\left(a_{w}\right) \geq c_{1} \rho\left(u, \Omega_{a_{w}}\right)
$$

para $\rho\left(u, \Omega_{a_{w}}\right)<\delta_{1}$ e tal que $u$ pertence à variedade

$$
M_{w}:=\left\{h \in X \mid F(h)=F\left(a_{w}\right)\right\} .
$$

Logo, baseados na desigualdade (3.16) podemos enunciar o seguinte teorema geral sobre a estabilidade de ondas viajantes periódicas para a equação (3.1a).

Teorema 3.1.1. (Estabilidade Orbital para a $C Q-S)$ Suponhamos que as hipóteses $H-1, H-2, H-3, H-4$ e $d^{\prime \prime}(w)>0$ para $w \in \Omega$ são verificadas. Então a órbita $\Omega_{a_{w}}$ gerada por $a_{w}$ é estável pelo fluxo periódico da equação CQ-S. Mais exatamente, dado $\epsilon>0$, existe $\delta(\epsilon)>0$ tal que para todo $u_{0} \in U_{\delta}$, a solução $u=u(t)$ do problema de valor inicial associado a CQ-S (3.1a)-(3.1b), satisfaz $u(t) \in U_{\epsilon}$ para todo $t \in \mathbb{R}$.

Demonstração. Para uma prova deste resultado ver Apêndice A.

\subsection{Positividade da transformada de Fourier e es- tabilidade}

Nesta seção vamos dedicar nossa atenção em desenvolver uma teoria geral que nos permita obter as propriedades espectrais dadas nos items H-3 e H-4 para os operadores lineares $\mathcal{L}_{R}, \mathcal{L}_{I m}$, dados em (3.12). Em [5] foram dadas condições suficientes para obter 
a estrutura espectral necessária para estudar estabilidade de ondas viajantes periódicas associadas a modelos gerais do tipo,

$$
u_{t}+u^{p} u_{x}-M u_{x}=0
$$

onde $M$ é um operador pseudo-diferencial definido via sua transformada de Fourier como

$$
\widehat{M g}(k)=\beta(k) \widehat{g}(k), \quad k \in \mathbb{Z} .
$$

O símbolo $\beta$ de $M$ é assumido ser mensurável, limitado localmente, par, e satisfazendo as condições

$$
A_{1}|k|^{m_{1}} \leqq|\beta(k)| \leqq A_{2}(1+|k|)^{m_{2}}
$$

com $1 \leqq m_{1} \leqq m_{2},|k| \geq k_{0}, \beta(k)>b$ para todo $k \in \mathbb{Z}$, e $A_{i}>0$. Embora equação (3.17) seja bastante geral, existem outros modelos para os quais o problema de estabilidade orbital de ondas periódicas gera operadores lineares cuja estrutura espectral requerida não é uma consequência da teoria desenvolvida em [5], por exemplo o operador $\mathcal{L}_{R}$ em (3.12). Por este motivo, nesta seção vamos considerar o seguinte modelo,

$$
u_{t}+f(u)_{x}-M u_{x}=0
$$

onde $M$ está definido em (3.18) e (3.19) e $f$ é uma função suave satisfazendo propriedades específicas determinadas abaixo. Observe que no caso $f(x)=\frac{x^{p+1}}{p+1}$ obtemos a equação (3.17), portanto os resultados que mostraremos aqui estendem aqueles obtidos em [5], embora as provas sejam muito similares. Um exemplo para $f$ que aparece nas aplicações é $f(x)=\lambda x^{p}+\mu x^{q}, \operatorname{com} p, q \in \mathbb{N}, p, q \geq 2$ e $\lambda, \mu \in \mathbb{R}$. A seguir, consideramos $a=a_{w}$ sendo uma solução positiva, par e periódica (período minimal $L$ ) para a equação pseudo-diferencial

$$
M a+w a-f(a)=k_{a}
$$

onde $k_{a}$ é uma constante real que será considerada igual a zero em nossa teoria. Equação (3.21) é obtida fazendo $u(x, t)=a(x-w t)$ em (3.20). Associado a equação (3.21) temos o operador linear $\mathcal{A}: D(\mathcal{A}) \rightarrow L_{\text {per }}^{2}([0, L])$ definido por,

$$
\mathcal{A}(u)=M u+w u-f^{\prime}(a) u,
$$


com $D(\mathcal{A})=H_{\text {per }}^{\frac{m_{2}}{2}}$. O intuito agora será apresentar as idéias desenvolvidas na teoria dada por Angulo\&Natali em [5] e que nos darão condições suficientes para obter a estrutura espectral requerida para o operador $\mathcal{A}$ em nosso estudo de estabilidade. O leitor pode verificar que as provas apresentadas por eles continuam sendo válidas neste caso. O primeiro resultado interessante sobre o operador $\mathcal{A}$ é o seguinte,

Teorema 3.2.1. (Propriedades espectrais gerais para $\mathcal{A})$. $\mathcal{A}$ é um operador auto-adjunto, fechado e o seu espectro está composto por uma sequência $\left\{\lambda_{i}\right\}_{i=0}^{\infty}$ de autovalores limitados inferiormente, satisfazendo,

$$
\lambda_{0} \leqslant \lambda_{1} \leqslant \lambda_{2} \cdots
$$

$e \lim _{i \rightarrow \infty} \lambda_{i}=\infty$. Em particular 0 é um autovalor com autofunção $\frac{d}{d x} a$.

Demonstração. Claramente $\mathcal{A}$ definido em (3.22) é um operador fechado, não limitado e auto-adjunto. Agora, vamos provar inicialmente que o espectro do operador $T:=M+w$ é um conjunto infinito e enumerável de autovalores satisfazendo

$$
\gamma_{0} \leq \gamma_{1} \leq \gamma_{2} \leq \cdots
$$

onde $\gamma_{n} \rightarrow \infty$ quando $n \rightarrow \infty$. De fato, seja $R_{w}:=(M+w)^{-1}$, cujo simbolo é $\frac{1}{w+\beta(n)}$ para $n \in \mathbb{Z}$. Como $\frac{1}{w+\beta(n)} \in l^{2}(\mathbb{Z})$ então existe um único $G_{w} \in L_{\text {per }}^{2}([0, L])$ tal que $\widehat{G_{w}}(n)=\frac{1}{w+\beta(n)}$. Devido a este fato tem-se a ação

$$
R_{w} f(x)=\frac{1}{L} \int_{0}^{L} G_{w}(x-y) f(y) d y
$$

definida em $L_{p e r}^{2}([0, L])$. Como o intervalo é limitado é possivel provar que o núcleo $\mathcal{G}_{w}(x, y)=G_{w}(x-y) \in L_{p e r}^{2}([0, L] \times[0, L])$, obtemos que $R_{w}$ é um operador compacto sobre $L_{\text {per }}^{2}([0, L])$ para todo $w>0$. Desta maneira, obtemos (3.23).

O proximo passo é mostrar que existe $\mu_{1}$ suficientemente grande de modo que $\Gamma=\left(\mathcal{A}+\mu_{1}\right)^{-1}$ existe e define um operador limitado, positivo, auto-adjunto e compacto. Com efeito, primeiramente é facil ver que o operador $\mathcal{A}$ é limitado por baixo, isto é existe $r_{0} \in \mathbb{R}$ tal que para todo $u \in D(\mathcal{A}),\langle\mathcal{A} u, u\rangle \geq r_{0}\|u\|_{L_{p e r}^{2}}^{2}$. Então, podemos 
escolher $\mu_{1}=: \mu$ tal que $\mathcal{A}+\mu_{1}>0$, em particular $\Gamma$ é positivo. Seja $\nu$ um número positivo tal que $\nu+f^{\prime}\left(a_{w}\right)-w>0$ e $\nu+\mu>0$. Assim, temos que

$$
u=(\mathcal{A}+\mu) g \Leftrightarrow(I-K) g=R_{\nu+\mu} u
$$

onde $K g=R_{\nu+\mu}\left[\left(\nu+f^{\prime}\left(a_{w}\right)-w\right) g\right]$. Agora, denotando $h=\nu+f^{\prime}\left(a_{w}\right)-w$ e usando o Teorema de Parseval, segue que

$$
\|K g\|_{L_{p e r}^{2}} \leq \sup _{n \in \mathbb{Z}}\left\{\frac{1}{\beta(n)+\nu+\mu}\right\}\|h\|_{L_{p e r}^{\infty}}\|g\|_{L_{p e r}^{2}} .
$$

Então, podemos escolher, $\mu$ tal que $\|K\|<1$ e $\mathcal{A}+\mu>0$. Portanto, $I-K$ é invertível e com isto $g=(I-K)^{-1} R_{\nu+\mu} u$. Assim escrevemos $\Gamma=(\mathcal{A}+\mu)^{-1}=(I-K)^{-1} R_{\nu+\mu}$. Sendo $R_{\nu+\mu}$ compacto e $(I-K)^{-1}$ limitado, deduzimos que $\Gamma$ é compacto. Logo, existe uma base ortonormal $\left\{\phi_{k}\right\}_{k=0}^{\infty}$ de $L_{\text {per }}^{2}$ constituida de autofunções de $\Gamma$ com autovalores não nulos $\left\{p_{k}\right\}_{k=0}^{\infty}$ satisfazendo,

$$
p_{1} \geq p_{2} \geq p_{3} \geq \cdots>0
$$

e $p_{k} \rightarrow 0$ quando $k \rightarrow \infty$. Como, $\mathcal{A} \phi_{k}=p_{k} \phi_{k} \in D(\mathcal{A}+\mu)$ obtemos

$$
\mathcal{A} \phi_{k}=\left(\frac{1}{\mu_{k}}-\mu\right) \phi_{k}:=\lambda_{k} \phi_{k} \text {. }
$$

Isto finaliza a prova.

Agora, definimos para $\theta \geq 0$, o operador $S_{\theta}: l^{2}(\mathbb{Z}) \rightarrow l^{2}(\mathbb{Z})$ para $\alpha=\left(\alpha_{j}\right)_{j \in \mathbb{Z}}$ como

$$
S_{\theta} \alpha(n)=\frac{1}{p_{\theta}(n)} \sum_{j=-\infty}^{\infty} K(n-j) \alpha_{j}=\frac{1}{p_{\theta}(n)}(K * \alpha)_{n},
$$

onde $p_{\theta}(n)=\beta(n)+\theta+w, K(n)=\widehat{g}(n) \operatorname{com} g(x):=f^{\prime}(a(x))$ e $n \in \mathbb{Z}$. Aqui escolhemos a velocidade $w$ da onda $a$ tal que $w>-b$ onde $b \in \mathbb{R}$ satisfaz $\beta(n)>b$ para todo $n \in \mathbb{Z}$. A seguir temos que o espaço $Y$ definido por

$$
Y=\left\{\alpha \in l^{2}(\mathbb{Z}) ;\|\alpha\|_{Y, \theta}:=\left(\sum_{n=-\infty}^{\infty}\left|\alpha_{n}\right|^{2} p_{\theta}(n)\right)^{\frac{1}{2}}\right\},
$$

é um espaço de Hilbert com norma $\|\cdot\|_{Y, \theta}$ e produto interno dado por $\langle\alpha, \gamma\rangle=$ $\sum_{n=-\infty}^{\infty} \alpha_{n} \overline{\gamma_{n}} p_{\theta}(n)$. A seguinte proposição é consequência da teoria de operadores autoadjuntos compactos. 
Proposição 3.2.1. Para todo $\theta \geq 0$, temos o seguinte,

(a) Se $\alpha \in l^{2}$ é uma autosequência de $S_{\theta}$ para um autovalor não nulo, então $\alpha \in Y$.

(b) A restrição de $S_{\theta}$ a $Y$ é compacta e auto-adjunta com respeito à norma $\|\cdot\|_{Y, \theta}$.

(c) 1 é um autovalor de $S_{\theta}$ (como operador de $Y$ ) se e só se $-\theta$ é um autovalor de $\mathcal{A}$ (como operador de $L_{\text {per }}^{2}$ ). Além disso, os dois autovalores tem a mesma multiplicidade.

(d) $S_{\theta}$ tem uma família de autosequências $\left(\psi_{i, \theta}\right)_{i=0}^{\infty}$, as quais formam uma base ortonormal de $Y$ com respeito à norma $\|\cdot\|_{Y, \theta}$. Os autovalores correspondentes $\left(\lambda_{i}(\theta)\right)_{i=0}^{\infty}$ tem como possível ponto de acumulação o zero e satisfazem, $\left|\lambda_{0}(\theta)\right| \geq\left|\lambda_{1}(\theta)\right| \geq$ $\left|\lambda_{2}(\theta)\right| \geq \cdots$.

Demonstração. Ver [5], para uma prova detalhada desta proposição. Aqui, observamos simplesmente que a condição de regularidade $\widehat{f^{\prime}(a)}(n) \in l^{1}(\mathbb{Z})$ é fundamental em tal prova.

Definição 3.2.1. (PF(2) caso discreto). Dado $\left\{p_{i}\right\}_{i \in \mathbb{Z}}$ Uma sequência $\left\{p_{i}\right\}_{i \in \mathbb{Z}}$ de números reais está em $P F(2)$ discreto se as seguintes condições são satisfeitas:

i) $p_{i}>0$, para todo $i \in \mathbb{Z}$.

ii) $p_{n_{1}-m_{1}} p_{n_{2}-m_{2}}-p_{n_{1}-m_{2}} p_{n_{2}-m_{1}}>0$, para todo $n_{1}, n_{2}, m_{1}, m_{2} \in \mathbb{Z}$, tal que $n_{1}<n_{2} e$ $m_{1}<m_{2}$.

Teorema 3.2.2. Sejam $\alpha$ e $\gamma$ duas sequências em PF(2) discreto, então a convolução $\alpha * \gamma \in P F(2)$ discreto (se a convolução faz sentido).

Demonstração. Ver Karlin [34].

Agora enunciamos o teorema que nos permite obter a estrutura espectral desejada para o operador $\mathcal{A}$ em (3.22).

Observação 3.2.1. Num contexto geral, a classe $P F(r)$ de funções de Freqüência Polya foi introduzida por I.J Shoenberg sob o nome $r$ vezes positiva e foi estudada em detalhe por S. Karlin em [34]. 
Teorema 3.2.3. Seja $a_{w}$ uma solução positiva par e periódica de (3.21) com $k_{a}=0$. Suponha que $\widehat{a_{w}}(n)>0$ para todo $n \in \mathbb{Z}$, e f é tal que $\left(\widehat{f^{\prime}\left(a_{w}\right)}(n)\right)_{n \in \mathbb{Z}} \in P F(2)$ discreto. Então $\mathcal{A}$ tem exatamente um único autovalor negativo o qual é simples, e zero é um autovalor simples com autofunção $\frac{d}{d x} a_{w}$.

Demonstração. A prova completa deste teorema é bastante técnica e longa, mas vamos fazer aqui uma exposição das idéias principais que estão envolvidas na prova (para mais detalhes referimos ao leitor a [5]). Para facilitar a compreensão da demonstração dividiremos esta em três passos.

I- Já que $S_{\theta}$ é um operador compacto autoadjunto sobre $Y$, segue que

$$
\lambda_{0}(\theta)= \pm \sup _{\|\alpha\|_{Y, \theta}=1}\left|\left\langle S_{\theta} \alpha, \alpha\right\rangle_{Y}\right|
$$

Seja $\psi(\theta):=\psi$ uma autosequência de $S_{\theta}$ correspondente a $\lambda_{0}(\theta):=\lambda_{0}$. Provaremos que $\psi$ não muda de sinal, isto é, $\psi(n) \geq 0$ ou $\psi(n) \leq 0 \forall n \in \mathbb{N}$. Suponhamos por contradição que $\psi$ toma valores positivos e negativos. Por hipótese o núcleo $\mathcal{K}=(\mathcal{K}(n))=\left(\widehat{f^{\prime}\left(a_{w}\right)}(n)\right)$ é positivo, então

$$
\begin{aligned}
S_{\theta}|\psi|(n) & =\frac{1}{p_{\theta}(n)} \sum_{j=-\infty}^{\infty} \mathcal{K}(n-j) \psi^{+}(j)+\frac{1}{p_{\theta}(n)} \sum_{j=-\infty}^{\infty} \mathcal{K}(n-j) \psi^{-}(j) \\
& >\left|\frac{1}{p_{\theta}(n)} \sum_{j=-\infty}^{\infty} \mathcal{K}(n-j) \psi^{+}(j)-\frac{1}{p_{\theta}(n)} \sum_{j=-\infty}^{\infty} \mathcal{K}(n-j) \psi^{-}(j)\right|,
\end{aligned}
$$

onde $\psi^{+}$e $\psi^{-}$são a parte positiva e negativa de $\psi$ respectivamente. Segue então que

$$
\left\langle S_{\theta}|\psi|,|\psi|\right\rangle_{Y, \theta}>\sum_{n=-\infty}^{\infty}\left|\lambda_{0}\right||\psi(n)|^{2} p_{\theta}(n)=\left|\lambda_{0}\right|\|\psi \mid\|_{Y, \theta}^{2} .
$$

Assumindo que $\|\psi\|_{Y}=1$, obtemos $\left\langle S_{\theta}|\psi|,|\psi|\right\rangle_{Y}>\left|\lambda_{0}\right|$, o qual contradiz (3.26). Portanto existe uma autosequência $\psi_{0}$ a qual é não negativa. Já que $\mathcal{K}$ é uma sequência positiva e $S_{\theta} \psi_{0}=\lambda_{0} \psi_{0}$, obtemos bentão que $\psi_{0}(n)>0$, para todo $n \in \mathbb{Z}$. Agora como, $\psi_{0}$ não pode ser ortogonal a qualquer outra autosequência não trivial em $Y$ associada ao autovalor $\lambda_{0}$ (pois pelo mostrado acima esta não 
mudaria de sinal), concluimos então que $\lambda_{0}$ é um autovalor simples. Notamos que o argumento anterior também mostra que $-\lambda_{0}$ não pode ser um autovalor de $S_{\theta}$, $\operatorname{logo},\left|\lambda_{1}\right|<\lambda_{0}$.

II- O próximo passo será estudar o comportamento do autovalor $\lambda_{1}(\theta)$. De fato, consideremos o seguinte conjunto de índices,

$$
\triangle=\left\{\left(n_{1}, n_{2}\right) \in \mathbb{Z} \times \mathbb{Z} ; n_{1}<n_{2}\right\} .
$$

Denotando $\bar{n}=\left(n_{1}, n_{2}\right)$ e $\bar{m}=\left(m_{1}, m_{2}\right)$, definimos para $\bar{n}, \bar{m} \in \Delta$ a seguinte sequência

$$
\mathcal{K}_{2}(\bar{n}, \bar{m}):=\mathcal{K}\left(n_{1}-m_{1}\right) \mathcal{K}\left(n_{2}-m_{2}\right)-\mathcal{K}\left(n_{1}-m_{2}\right) \mathcal{K}\left(n_{2}-m_{1}\right) .
$$

Por hipótese $\mathcal{K} \in P F(2)$ discreto, $\operatorname{logo} \mathcal{K}_{2}>0$. Seja $l^{2}(\triangle)$ definido como:

$$
l^{2}(\Delta)=\left\{\alpha=\left(\alpha_{\bar{n}}\right)_{\bar{n} \in \Delta} ; \sum \sum_{\Delta}\left|\alpha_{\bar{n}}\right|^{2}:=\sum_{n_{1} \in \mathbb{Z}} \sum_{\substack{n_{1}<n_{2} \\ n_{2} \in \mathbb{Z}}}\left|\alpha\left(n_{1}, n_{2}\right)\right|^{2}<+\infty\right\},
$$

e definamos o operador $S_{2, \theta}: l^{2}(\triangle) \rightarrow l^{2}(\triangle)$ por

$$
S_{2, \theta} g(\bar{n})=\sum \sum_{\Delta} G_{2, \theta}(\bar{n}, \bar{m}) g(\bar{m})
$$

onde $G_{2, \theta}(\bar{n}, \bar{m})=\frac{\mathcal{K}_{2}(\bar{n}, \bar{m})}{p_{\theta}\left(n_{1}\right) p_{\theta}\left(n_{2}\right)}$. Consideremos também o espaço

$$
W=\left\{\alpha \in l^{2}(\triangle) ;\|\alpha\|_{W, \theta}:=\left(\sum \sum_{\Delta}|\alpha(\bar{n})|^{2} p_{\theta}\left(n_{1}\right) p_{\theta}\left(n_{2}\right)\right)^{\frac{1}{2}}<\infty\right\} .
$$

Então $W$ é um espaço de Hilbert com norma $\|\cdot\|_{W, \theta}$ dada acima e com produto interno dado por

$$
\left\langle\alpha^{1}, \alpha^{2}\right\rangle_{W, \theta}=\sum \sum_{\Delta} \alpha^{1}(\bar{n}) \overline{\alpha^{2}(\bar{n})} p_{\theta}\left(n_{1}\right) p_{\theta}\left(n_{2}\right) .
$$

Nota 3.2.1. 1) Analogamente a Proposição 3.2.1, é possivel mostrar que $\left.S_{2, \theta}\right|_{W}$ é um operador compacto e autoadjunto. Portanto, os autovalores associados a este operador podem ser enumerados em ordem decrescente em valor absoluto, assim, $\left|\mu_{0}(\theta)\right| \geq\left|\mu_{1}(\theta)\right| \geq\left|\mu_{2}(\theta)\right| \geq \cdots$.

2) Também $\mu_{0}(\theta):=\mu_{0}$ é um autovalor positivo simples e $\left|\mu_{1}\right|<\mu_{0}$. 
Agora introduzimos a seguinte definição

Definição 3.2.2. Seja $\alpha^{1}, \alpha^{2} \in l^{2}(\mathbb{Z})$, definimos o produto wedge $\alpha^{1} \wedge \alpha^{2}$ em $\triangle$ por $\left(\alpha^{1} \wedge \alpha^{2}\right)\left(n_{1}, n_{2}\right)=\alpha^{1}\left(n_{1}\right) \alpha^{2}\left(n_{2}\right)-\alpha^{1}\left(n_{2}\right) \alpha^{2}\left(n_{1}\right)$.

Com esta definição temos os seguintes resultados.

Lema 3.2.1. 1) Seja $A=\left\{\alpha^{1} \wedge \alpha^{2}\right.$; para $\left.\alpha^{1}, \alpha^{2} \in Y, \alpha^{1} \wedge \alpha^{2} \in l^{2}(\triangle)\right\}$. Então $A$ é denso em $W$.

2) Seja $\alpha^{1}, \alpha^{2} \in l^{2}(\mathbb{Z})$. Então $S_{2, \theta}\left(\alpha^{1} \wedge \alpha^{2}\right)=S_{\theta} \alpha^{1} \wedge S_{\theta} \alpha^{2}$.

Demonstração. Albert [2] e Karlin [34].

Sabemos que $\lambda_{0}$ é um autovalor isolado de $S_{\theta}^{Y}$. Isto nos fornece (ver [35]) uma partição do espectro de $S_{\theta}^{Y}$ em $\sum_{\theta}^{0}$ e $\sum_{\theta}^{1}$. Consequentemente obtemos uma decomposição do espaço $Y$ em $Y=M_{0} \oplus N$, onde $M_{0}=\left[\psi_{0}\right]$, de tal maneira que o espectro das partes $\left.S_{\theta}^{Y}\right|_{M_{0}},\left.S_{\theta}^{Y}\right|_{N}$ coincidem com $\sum_{\theta}^{0}$ e $\sum_{\theta}^{1}$ respectivamente. $M_{0}$ em verdade determina a projeção ortogonal $P_{\theta}$ de $Y$ sobre o autoespaço associado à $\lambda_{0}$ e $N=\operatorname{Ker}(P)$. Tem-se ainda a decomposição de $S_{\theta}^{Y}$ da forma

$$
S_{\theta}^{Y}=\lambda_{0} P_{\theta}+Q_{\theta}
$$

onde $Q_{\theta}:=S_{\theta}^{Y}-\lambda_{0} P_{\theta}$ e se satisfazem $P_{\theta} Q_{\theta}=Q_{\theta} P_{\theta}=0$ e $\left.Q_{\theta}\right|_{N}=\left.S_{\theta}^{Y}\right|_{N}: N \rightarrow N$ (pois, $\left.Q_{\theta}\right|_{N} \equiv 0$ ). Ademais, ainda pela teoria espectral contida em Kato [35] o autovalor $\lambda_{0}=\lambda_{\theta}$ é diferenciavel com relação à $\theta \geq 0$ bem como $\mu_{0}=\mu_{\theta}$. Como consequência $\psi_{0}$ e $\tau_{0}=$ autosequência associada a $\mu_{0}=\mu_{\theta}$ são autosequências diferenciáveis em relação a $\theta$. Com isto temos o seguinte lema.

Lema 3.2.2. Na notação dada anteriormente temos,

a)

$$
\frac{\left(S_{\theta}^{Y}\right)^{m}}{\lambda_{0}^{m}} \rightarrow P_{\theta},
$$

quando $m \rightarrow \infty$ na topologia forte de $B(Y, Y)$. 
b) Um resultado análogo ao item a) vale para $S_{2, \theta}$ no lugar de $S_{\theta}$, $\mu_{0}$ no lugar de $\lambda_{0}$ e a projeção ortogonal correspodente no lugar de $P_{\theta}$.

Demonstração. Ver [5].

O seguinte lema é a chave na caracterização do segundo autovalor $\lambda_{1}$.

Lema 3.2.3. Para todo $\theta \geq 0$ temos:

1- $\mu_{0}(\theta)=\lambda_{0}(\theta) \lambda_{1}(\theta)$, e obviamente $\lambda_{1}(\theta)>0$.

2- $\lambda_{1}(\theta)$ é simples.

Demonstração. 1- Temos pelo Lema 3.2.1, $\lambda_{0} \lambda_{1}$ é autovalor de $S_{2, \theta}$ com autosequência $\psi_{0} \wedge \psi_{1}$, onde $\psi_{1}(\theta):=\psi_{1}$ é a autosequência associada a $\lambda_{1}$. De fato,

$$
S_{2, \theta}\left(\psi_{0} \wedge \psi_{1}\right)=S_{\theta} \psi_{0} \wedge S_{\theta} \psi_{1}=\lambda_{0} \lambda_{1}\left(\psi_{0} \wedge \psi_{1}\right)
$$

Assim, $\mu_{0} \geq \lambda_{0}\left|\lambda_{1}\right|$. O resto da prova deste item segue-se de um argumento por contradição ao item b) no lema 3.2.2. De fato, se $\mu_{0}>\lambda_{0}\left|\lambda_{1}\right|$ é possivel concluir que

$$
\frac{\left(S_{2, \theta}^{Y}\right)^{m}}{\mu_{0}^{m}} \rightarrow 0 .
$$

(Ver detalhes em [5]). Isto completa a prova deste item.

2- Em [5] é mostrado que qualquer autosequência $\psi_{1}$ associada ao autovalor $\lambda_{1}$ é impar e satisfaz $\psi(n)>0$ para todo $n \in \mathbb{N}$, alem disso temos que

$$
\psi(n)=0 \Leftrightarrow n=0
$$

Estas propriedades mostram que duas sequências deste tipo não podem ser ortogonais pois o produto delas é par e portanto $\lambda_{1}$ é simples. 
III- Passo final. Para $i=0,1$, temos que a curva diferenciável $\theta \rightarrow \lambda_{i}(\theta)$ satisfaz $\frac{d}{d \theta} \lambda_{i}(\theta)<0$ e $\lim _{\theta \rightarrow \infty} \lambda_{0}(\theta)=0$. De $\widehat{a_{w}}(n)>0$ para todo $n \in \mathbb{Z}$, segue que $\lambda_{1}(0)=1$. Usando então a Proposição 3.2.1, obtemos que $\kappa \equiv-\theta_{0}$ é um autovalor negativo de $\mathcal{A}$ o qual é simples. Para $i \geq 2$ e $\theta>0$ temos que

$$
\lambda_{i}(\theta) \leq \lambda_{1}(\theta)<\lambda_{1}(0)
$$

$\operatorname{logo} 1$ não pode ser autovalor de $S_{\theta}$ para $\theta \in(0, \infty) \backslash\left\{\theta_{0}\right\}$, pois 1 é um autovalor só para $\theta=0$ e $\theta=\theta_{0}$. Assim $\mathcal{A}$ tem um único autovalor negativo e simples. Finalmente, como $\lambda_{1}(0)=1$ e $\lambda_{1}$ é um autovalor simples segue da Proposição 3.2.1 que $\theta=0$ é um autovalor simples de $\mathcal{A}$. Isto finaliza a prova do Teorema.

Nos seguintes capítulos usaremos o Teorema 3.2.3 para mostrar nossos resultados de estabilidade orbital de ondas viajantes periódicas positivas para diferentes tipos de equações dispersivas não lineares. 


\section{CAPÍTULO 4}

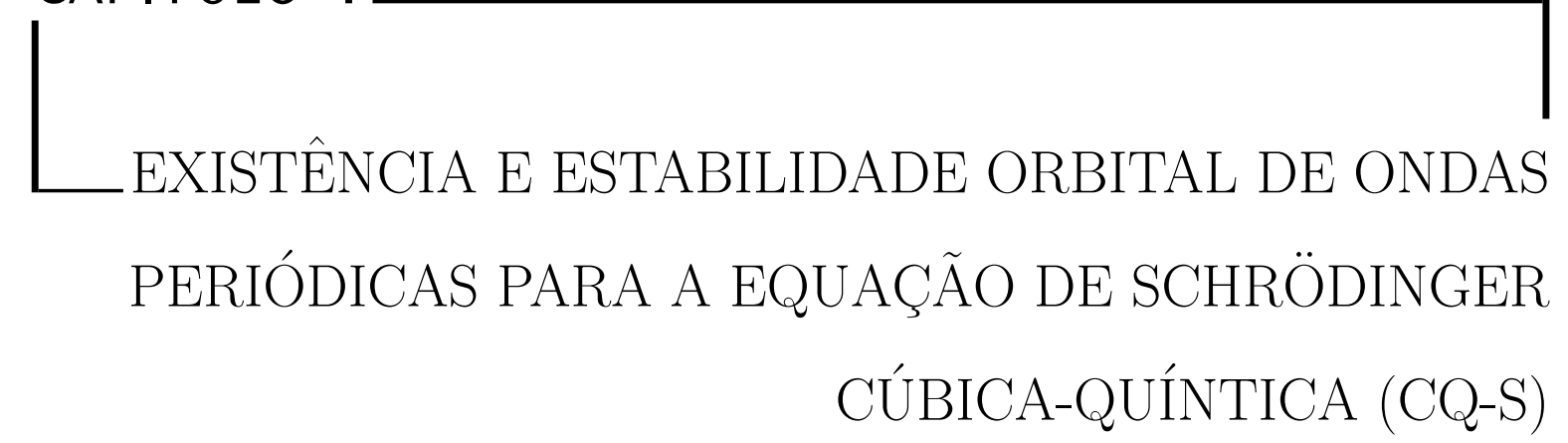

Neste capítulo entenderemos alguns aspectos sobre a dinâmica da equação de Schrödinger cúbica-quíntica (CQ-S)

$$
i u_{t}+u_{x x}+u\left(|u|^{2}+|u|^{4}\right)=0 .
$$

Mais especificamente, na seção 4.1 estudaremos a boa colocação local e global do problema de Cauchy associado a CQ-S. A existência de uma curva de standing waves periódicas para a CQ-S será estudado na Seção 4.2. As propriedades espectrais dos operadores $\mathcal{L}_{R}$ e $\mathcal{L}_{I m}$ dados em (3.12) serão estudadas na Seção 4.3. A convexidade da função $d$ definida em (3.13) será abordada na Seção 4.4. Um resultado de instabilidade de standing waves por perturbações subharmônicas será mostrado na Seção (4.5). Na Seção 4.6 mostraremos a estabilidade orbital de soluções da CQ-S de tipo standing waves com perfil constante. Finalmente mostraremos como é possível aplicar os resultados de existência e estabilidade obtidos para a CQ-S na equação MC-KdV (4.121). 
A seguir, iniciamos nosso estudo de estabilidade mostrando primeiramente os resultados de boa colocação de tipo local e global para a CQ-S no espaço de energia $H_{p e r}^{1}([0, L])$. Como foi observado no capítulo anterior estes resultados são fundamentais no estudo de um problema de estabilidade.

\subsection{Boa colocação local e global para a equação CQ- S}

A boa colocação do problema de Cauchy periódico associado com a CQ-S será estudada nesta seção, mais exatamente, estamos interessados na existência, unicidade e depêndencia contínua sob o dado inicial $\phi$ para o problema de valor inicial

$$
\begin{aligned}
& i u_{t}+u_{x x}=G(u), \quad(x, t) \in \mathbb{R} \times \mathbb{R}, \\
& u(x, 0)=\phi(x) \in H_{p e r}^{s}([0, L]),
\end{aligned}
$$

com $G(u)=-u\left(|u|^{2}+|u|^{4}\right)$. Nosso intuito nesta parte será dar as idéias expostas no capítulo 5 de Iório\&Iório [31], onde a boa colocação local do problema (4.2) é estudada sob hipóteses gerais dadas na função $G$. A saber

i) GNLS1 $G: H_{p e r}^{s} \rightarrow H_{p e r}^{s}$ e $G(0)=0$.

ii) GNLS2 $G$ é localmente Lipschitz, isto é

$$
\|G(z)-G(w)\|_{s} \leq L\left(\|z\|_{s},\|w\|_{s}\right)\|z-w\|_{s}, \quad \forall z, w \in H_{p e r}^{s}
$$

onde $L(\cdot, \cdot)$ é uma função continua e não decrescente com respeito a cada um de seus argumentos. Em particular

$$
\|G(z)\|_{s} \leq L\left(\|z\|_{s}, 0\right)\|z\|_{s}, \quad \forall z \in H_{p e r}^{s}
$$

Lema 4.1.1. Sejam $s>\frac{1}{2}$ e $u \in H_{\text {per }}^{s}$. Então a função $G$ definida por $G(u)=$ $u\left(|u|^{2}+|u|^{4}\right)$ satisfaz as condições $\boldsymbol{G N L S 1 ~ e ~} \boldsymbol{G N L S 2 ~ a c i m a . ~}$ 
Demonstração. Obviamente $G(0)=0$. Agora, como para $s>\frac{1}{2}, H_{\text {per }}^{s}$ é uma álgebra de Banach, então deduzimos que

$$
\left\||u|^{2 k} u\right\|_{s}=\left\|u^{k+1} \bar{u}^{k}\right\|_{s} \leq\|u\|_{s}^{k+1}\|\bar{u}\|_{s}^{k}=\|u\|_{s}^{2 k+1}
$$

para $k=1,2,3, \ldots$ Assim $G$ está bem definida como função de $H_{p e r}^{s}$ em $H_{\text {per }}^{s}$, o que mostra que $G$ satisfaz a condição GNLS1. Por outro lado observamos que para $p, q \in \mathbb{C}$ temos as seguintes identidades

$$
|p|^{2} p-|q|^{2} q=|p|^{2}(p-q)+p q \overline{(p-q)}+|q|^{2}(p-q)
$$

e

$$
\begin{aligned}
|p|^{4} p-|q|^{4} q= & |p|^{4}(p-q)+p q|p|^{2} \overline{(p-q)}+|p|^{2}|q|^{2}(p-q) \\
& +p q|q|^{2} \overline{(p-q)}+|q|^{4}(p-q) .
\end{aligned}
$$

Agora para $z, w \in H_{p e r}^{s}$, deduzimos de (4.6) e (4.7) que

$$
\begin{aligned}
& |||z|^{2} z-|w|^{2} w\left\|_{s} \leq L_{1}\left(\|z\|_{s},\|w\|_{s}\right)\right\| z-w \|_{s} \\
& \left|\left\|\left.z\right|^{4} z-|w|^{4} w\right\|_{s} \leq L_{2}\left(\|z\|_{s},\|w\|_{s}\right)\|z-w\|_{s}\right.
\end{aligned}
$$

com

$$
L_{1}(x, y)=x^{2}+x y+y^{2}
$$

e

$$
L_{2}(x, y)=x^{4}+x^{3} y+x^{2} y^{2}+x y^{3}+y^{4} .
$$

Portanto, usando (4.8), (4.9) e (4.10) obtemos que

$$
\|G(z)-G(w)\|_{s} \leq L\left(\|z\|_{s},\|w\|_{s}\right)\|z-w\|_{s}
$$

onde $L(x, y)=L_{1}(x, y)+L_{2}(x, y)$ satisfaz as condições de continuidade e crescimento dadas em GNLS2. Isto conclui a prova do lema.

Observação 4.1.1. Para $s>\frac{1}{2}$, é possível mostrar que as funções $G_{k}(p):=|p|^{k} p$, $k \in \mathbb{N}, p \in \mathbb{C}$, induzem aplicações $G_{k}: H_{\text {per }}^{s} \rightarrow H_{\text {per }}^{s}$ satisfazendo as condições $\boldsymbol{G N L \boldsymbol { S } \mathbf { 1 }}$ e GNLS2. 
No que segue, assumiremos a não linearidade $G$ satisfazendo as condições GNLS1 e GNLS2 dadas acima. Continuando com a questão de existência e unicidade de soluções para o problema (4.2), vamos considerar agora a fórmula de variação dos parametros associada ao problema (4.2), isto é, a fórmula de Duhamel,

$$
u(t)=U_{0}(t) \phi-i \int_{0}^{t} U_{0}(t-y) G(u(y)) d y
$$

onde $U_{0}$ denota o grupo de operadores unitarios $U_{0}(t): H_{\text {per }}^{s} \rightarrow H_{\text {per }}^{s}, \forall s \in \mathbb{R}$, definido por

$$
U_{0}(t)=e^{i t \partial_{x}^{2}}
$$

Observamos agora que os problemas (4.2) e (4.12) são equivalentes. Mais exatamente temos o seguinte Lema:

Lema 4.1.2. Seja $u \in C\left([0, T] ; H_{p e r}^{s}\right)$ uma solução do problema (4.2). Então u satisfaz (4.12). Reciprocamente, se $u \in C\left([0, T] ; H_{\text {per }}^{s}\right)$ é uma solução de (4.12), então $u \in$ $C^{1}\left([0, T] ; H_{\text {per }}^{s-2}\right)$ e satisfaz (4.2), com a derivada com respeito de $t$ calculada no seguinte sentido:

$$
\lim _{h \rightarrow 0}\left\|\frac{u(t+h)-u(t)}{h}-i\left(\partial_{x}^{2} u(t)-G(u(t))\right)\right\|_{s-2}=0 .
$$

Demonstração. Ver [31] Proposição 5.1.

Agora, sobre a unicidade de soluções para o problema (4.2) temos

Lema 4.1.3. Seja $s>\frac{1}{2}, \phi, \psi \in H_{\text {per }}^{s}$ e $u, v \in C\left([0, T] ; H_{\text {per }}^{s}\right)$ duas soluções do problema (4.2) satisfazendo $u(0)=\phi$ e $v(0)=\psi$. Então

$$
\|u(t)-v(t)\|_{s} \leq\|\phi-\psi\|_{s} e^{L\left(M_{s}, M_{s}\right) t}, \quad \forall t \in[0, T]
$$

onde $M_{s}$, é dado por

$$
M_{s}=M_{s}(u, v)=\max \left\{\sup _{[0, T]}\|u(t)\|_{s}, \sup _{[0, T]}\|v(t)\|_{s}\right\},
$$

e L é definido na prova do Lema 4.1.1. Em particular (4.2) tem no máximo uma solução. 
Demonstração. Da equação (4.12) temos que

$$
u(t)-v(t)=U_{0}(t)(\phi-\psi)-i \int_{0}^{t} U_{0}(t-y)[G(u(y))-G(v(y))] d y .
$$

Como $U_{0}(t)$ é unitario em $H_{p e r}^{s}$ segue então que

$$
\begin{aligned}
\|u(t)-v(t)\|_{s} & \leq\|\phi-\psi\|_{s}+\int_{0}^{t}\|G(u(y))-G(v(y))\|_{s} d y \\
& \leq\|\phi-\psi\|_{s}+\int_{0}^{t} L\left(\|u(y)\|_{s},\|v(y)\|_{s}\right)\|u(y)-v(y)\|_{s} d y \\
& \leq\|\phi-\psi\|_{s}+L\left(M_{s}, M_{s}\right) \int_{0}^{t}\|u(y)-v(y)\|_{s} d y
\end{aligned}
$$

o resultado segue da desigualdade de Gronwall (ver Teorema A.3 em [31]).

Agora o nosso objetivo é provar existência de soluções.

Teorema 4.1.1. Seja $\phi \in H_{\text {per }}^{s}, s>\frac{1}{2}$. Então existe um $T>0$ e uma única $u \in$ $C\left([0, T] ; H_{p e r}^{s}\right)$ satisfazendo (4.2) com a derivada calculada na forma (4.14).

Demonstração. Seja $\gamma>0$ fixado. Pode ser provado que o conjunto

$$
\Lambda(T, \gamma, \phi)=\left\{v \in C\left([0, T] ; H_{p e r}^{s}\right) \mid\left\|v(t)-U_{0}(t) \phi\right\| \leq \gamma, \forall t \in[0, T]\right\}
$$

junto com a métrica

$$
d(u, v)=\sup _{[0, T]}\|u(t)-v(t)\|_{s}
$$

é um espaço métrico completo. Note que $\Lambda(T, \gamma, \phi) \neq \emptyset$ pois $v(t)=U_{0}(t) \phi \in$ $C\left(\mathbb{R} ; H_{\text {per }}^{s}\right)$. Agora, consideramos a aplicação $A: C\left([0, T] ; H^{s}\right) \rightarrow C\left([0, T] ; H^{s}\right)$

$$
(A v)(t)=U_{0}(t) \phi-i \int_{0}^{t} U_{0}(t-y) G(u(y)) d y .
$$

É facil verificar que $A v \in C\left([0, T] ; H_{p e r}^{s}\right)$ para todo $v \in \Lambda(T, \gamma, \phi)$ e $T>0$. Mostraremos que existe $T>0$ tal que $A: \Lambda(T, \gamma, \phi) \rightarrow \Lambda(T, \gamma, \phi)$ é uma contração. Primeiramente combinando o fato $U_{0}(t)$ é unitário em $H_{\text {per }}^{s}$ e a desigualdade (4.4), obtemos que

$$
\begin{aligned}
\left\|(A v)(t)-U_{0}(t) \phi\right\|_{s} & \leq \int_{0}^{t}\|G(v(y))\|_{s} d y \\
& \leq \int_{0}^{t} L\left(\|v(y)\|_{s}, 0\right) \|\left(v(y) \|_{s} d y\right.
\end{aligned}
$$


como $v \in \Lambda(T, \gamma, \phi)$, temos que

$$
\|v(y)\| \leq\left\|v(y)-U_{0}(y) \phi\right\|+\left\|U_{0}(y) \phi\right\| \leq \gamma+\|\phi\|_{s}, \quad \forall y \in[0, T] .
$$

Assim, deduzimos que

$$
\left\|(A v)(t)-U_{0}(t) \phi\right\|_{s} \leq L\left(\gamma+\|\phi\|_{s}, 0\right)\left(\gamma+\|\phi\|_{s}\right) T, \quad \forall t \in[0, T] .
$$

Segue então que $A v \in \Lambda(T, \gamma, \phi)$ sempre que

$$
0<T \leq\left[L\left(\gamma+\|\phi\|_{s}, 0\right)\left(\gamma+\|\phi\|_{s}\right)\right]^{-1} \gamma=\alpha\left(\gamma,\|\phi\|_{s}\right)
$$

Por outro lado consideremos $v, w \in \Lambda(T, \gamma, \phi)$. Então

$$
\begin{aligned}
\|(A v)(t)-(A w)(t)\|_{s} & \leq \int_{0}^{t}\|G(v(y))-G(w(y))\|_{s} d y \\
& \leq \int_{0}^{t} L\left(\|v(y)\|_{s},\|w(y)\|_{s}\right)\|v(y)-w(y)\|_{s} d y \\
& \leq L\left(\gamma+\|\phi\|_{s}, \gamma+\|\phi\|_{s}\right) \int_{0}^{t}\|v(y)-w(y)\|_{s} d y \\
& \leq L\left(\gamma+\|\phi\|_{s}, \gamma+\|\phi\|_{s}\right) T d(v, w),
\end{aligned}
$$

para todo $t \in[0, T]$. Logo

$$
d(A v, A w) \leq L\left(\gamma+\|\phi\|_{s}, \gamma+\|\phi\|_{s}\right) T d(v, w) .
$$

O fator multiplicando $d(v, w)$ é menor que 1 se escolhemos $T$ tal que

$$
0<T<L\left(\gamma+\|\phi\|_{s}, \gamma+\|\phi\|_{s}\right)^{-1}=\beta\left(\gamma,\|\phi\|_{s}\right)
$$

Portanto de (4.25) e (4.28), podemos concluir que $A$ é uma contração em $\Lambda(T, \gamma, \phi)$ sempre que escolhamos $T$ na forma

$$
0<T<T^{\prime}=T^{\prime}\left(\gamma,\|\phi\|_{s}\right)=\min \left\{\alpha\left(\gamma,\|\phi\|_{s}\right), \beta\left(\gamma,\|\phi\|_{s}\right)\right\}
$$

Isto conclui a prova do teorema.

O seguinte teorema mostra como a solução $u \in C\left([0, T] ; H_{p e r}^{s}\right)$ do problema (4.2) depende continuamente do dado inicial $\phi$. 
Teorema 4.1.2. Seja $\phi_{n} \in H_{p e r}^{s}, n=1,2, \ldots, \infty$ tal que $\phi_{n} \rightarrow \phi_{\infty}$ em $H_{p e r}^{s}, s>$ $\frac{1}{2}$. Sejam $u_{n} \in C\left(\left[0, T_{n}^{\prime}\right) ; H_{\text {per }}^{s}\right)$, onde $T_{n}^{\prime}=T_{n}^{\prime}\left(\gamma,\left\|\phi_{n}\right\|_{s}\right)$, as soluções construidas no Teorema 4.1.1. Seja $T \in\left(0, T_{\infty}^{\prime}\right)$. Então as soluções $u_{n}$ podem ser definidas em $[0, T]$ para todo $n$ suficientemente grande $e$

$$
\lim _{n \rightarrow \infty} \sup _{[0, T]}\left\|u_{n}(t)-u_{\infty}(t)\right\|_{s}=0
$$

Demonstração. Como $T^{\prime}\left(\gamma,\|\phi\|_{s}\right)$ é uma função continua de $\phi$, então existe $N \in \mathbb{N}$ tal que $T_{n}^{\prime}>T$, para todo $n \geq N$. Portanto as $u_{n}$ estão definidas em $[0, T]$ para $n \geq N$. Logo segue que as $u_{n} \in \Lambda\left(T, \gamma, \phi_{n}\right)$ satisfazem

$$
\left\|u_{n}(t)\right\|_{s} \leq\left\|\phi_{n}\right\|_{s}+\gamma \leq K+\gamma
$$

onde $K=\sup _{n}\left\|\phi_{n}\right\|_{s}$. Agora, combinando Lema 4.1.3 com (4.31) deduzimos que

$$
\left\|u_{n}(t)-u_{\infty}(t)\right\|_{s} \leq\left\|\phi_{n}-\phi_{\infty}\right\|_{s} e^{K_{s, n} T}
$$

onde

$$
K_{s, n}=L\left(M_{s}\left(u_{n}, u_{\infty}\right), M_{s}\left(u_{n}, u_{\infty}\right)\right) \leq L(K+\gamma, K+\gamma)=K^{\prime}<\infty .
$$

De (4.32) e (4.33) obtemos então que

$$
\left\|u_{n}(t)-u_{\infty}(t)\right\|_{s} \leq\left\|\phi_{n}-\phi_{\infty}\right\|_{s} e^{K^{\prime} T} . \forall t \in[0, T] .
$$

Como queríamos provar.

Agora introduziremos o conceito de boa colocação local.

Definição 4.1.1. Sejam $X, Y$ espaços de Banach, $T_{0} \in(0, \infty)$ e seja $F:\left[0, T_{0}\right] \times Y \rightarrow$ $X$ uma função contínua. Dizemos que o problema de Cauchy

$$
\left\{\begin{array}{l}
\partial_{t} u(t)=F(t, u(t)) \in X, \\
u(0)=\phi \in Y,
\end{array}\right.
$$

é localmente bem posto em $Y$ se 
(a) Existe $T \in\left(0, T_{0}\right]$ e uma função $u \in C([0, T], Y)$ tal que $u(0)=\phi$ e a equação diferencial é satisfeita no sentido que

$$
\lim _{h \rightarrow 0}\left\|\frac{u(t+h)-u(t)}{h}-F(t, u(t))\right\|_{X}=0
$$

aqui a derivada em $t=0$ e $t=T$ são calculadas pela direita e pela esquerda respectivamente.

(b) O problema (4.35) tem no máximo uma solução em $C([0, T] ; Y)$.

(c) A aplicação $\phi \rightarrow u$ é contínua. Mais precisamente, sejam $\phi_{n} \in Y, n=1,2, \ldots, \infty$ tal que $\phi_{n} \rightarrow \phi_{\infty}$ na topologia de $Y$, e sejam $u_{n} \in C\left(\left[0, T_{n}\right] ; Y\right)$ as soluções correspondentes. Seja $T \in\left(0, T_{\infty}\right)$. Então as soluções $u_{n}$ estão definidas em $[0, T]$ para todo $n$ suficientemente grande e

$$
\lim _{n \rightarrow \infty} \sup _{[0, T]}\left\|u_{n}(t)-u_{\infty}(t)\right\|_{Y}=0 .
$$

Se alguma destas condições não for satisfeita dizemos que o problema (4.35) é mau colocado.

Agora estamos en condições de enunciar o seguinte teorema:

Teorema 4.1.3. O problema de Cauchy associado a equação CQ-S (4.1) é localmente bem posto no espaço $H_{\text {per }}^{s}([0, L])$, para $s>\frac{1}{2}$.

Demonstração. A prova segue dos Lemas 4.1.1, 4.1.2 e 4.1.3 e dos Teoremas 4.1.1 e 4.1.2

Observação 4.1.2. O Teorema 4.1.3 continua sendo valido se mudamos a não linearidade $G$ em (4.2) pela função $G_{0}(u)=-u\left(\lambda|u|^{2}+\mu|u|^{4}\right)$, onde $\lambda, \mu \in \mathbb{R}$. De fato, não é difícil ver que $G_{0}$ também satisfaz as hipoteses $\boldsymbol{G N L S 1 ~ e ~} \boldsymbol{G N L S 2 ~ a c i m a . ~}$

Agora, estamos interessados na questão da boa colocação global para o problema de Cauchy associado com a CQ-S (4.1). Isto é, queremos saber se é possível considerar 
a solução $u$ do problema (4.2) definida para todo tempo $t$, ou seja $u \in C\left([0, \infty) ; H_{p e r}^{s}\right)$. Para este fim consideremos $\phi \in H_{p e r}^{s}$ e definamos

$$
T^{*}(\phi)=\sup \{T>0 \mid \exists ! \text { solução de }(4.2) \text { em }[0, T]\}
$$

então obtemos o seguinte resultado o qual pode ser encontrado em [31]

Teorema 4.1.4. Sejam $s>\frac{1}{2}$ e $\phi \in H_{p e r}^{s}([0, L])$. Então $T^{*}(\phi)=\infty$ ou $T^{*}(\phi)<\infty e$

$$
\lim _{t \rightarrow T^{*}}\|u(t)\|_{s}=\infty
$$

Demonstração. Ver Teorema 5.14 em [31].

Notamos aqui que a boa colocação local estabelecida no Teorema 4.1.3 assim como as condições GNLS1 e GNLS2 definidas acima, são parte importante na prova do Teorema 4.1.4.

Teorema 4.1.5. Seja $\phi \in H_{p e r}^{1}([0, L])$. Então a solução u do problema (4.2) é globalmente definida sempre que a norma do dado inicial $u(0)=\phi$ seja pequena no espaço $L_{\text {per }}^{2}([0, L])$.

Demonstração. Do Teorema 4.1.4 basta mostrar que a norma $\|u(t)\|_{1} \operatorname{com} t \in\left[0, T^{*}(\phi)\right)$ é uniformemente limitada. Primeiro observamos que a desigualdade de GagliardoNirenberg nos permite obter uma estimativa das norma $\|u\|_{L^{4}}$ e $\|u\|_{L^{6}}$, da seguinte maneira

$$
\begin{aligned}
& \|u\|_{L^{6}} \leq c\left\|u_{x}\right\|_{L^{2}}^{\frac{1}{3}}\|u\|_{L^{2}}^{\frac{2}{3}}, \quad \mathrm{e} \\
& \|u\|_{L^{4}} \leq d\left\|u_{x}\right\|_{L^{2}}^{\frac{1}{4}}\|u\|_{L^{2}}^{\frac{3}{4}},
\end{aligned}
$$

onde $c$ e $d$ são constantes positivas. De (4.40) deduzimos que

$$
\begin{aligned}
& \|u\|_{L^{6}}^{6} \leq c_{0}\left\|u_{x}\right\|_{L^{2}}^{2}\|u\|_{L^{2}}^{4} \quad \mathrm{e} \\
& \|u\|_{L^{4}}^{4} \leq d_{0}\left\|u_{x}\right\|_{L^{2}}\|u\|_{L^{2}}^{3},
\end{aligned}
$$

com $c_{0}=c^{6}$ e $d_{0}=d^{4}$. Da segunda desigualdade em (4.41) podemos obter que:

$$
\|u\|_{L^{4}}^{4} \leq \frac{d_{0}}{2}\left(\left\|u_{x}\right\|_{L^{2}}^{2}+\|u\|_{L^{2}}^{6}\right)
$$


Agora, vamos a usar as estimativas dadas em (4.41) e (4.42), assim como as quantidades conservadas $E$ e $F$ definidas em (3.6) para mostrar que a solução $u$ associada ao problema (4.2) com dado inicial $u(0)=\phi \in H_{\text {per }}^{1}([0, L])$ é uniformemente limitada sempre que a norma $L_{\text {per }}^{2}$ do dado inicial $\phi$ seja pequena. Como a norma $L^{2}$ é conservada, então basta mostrar a limitação uniforme da quantidade $\left\|u_{x}\right\|_{L^{2}}^{2}$. Assim:

$$
\begin{aligned}
\left\|u_{x}(t)\right\|_{L^{2}}^{2} & =2 E(u)+\frac{1}{2}\|u\|_{L^{4}}^{4}+\frac{1}{3}\|u\|_{L^{6}}^{6} \\
& \leq 2 E(u)+\frac{d_{0}}{4}\left(\left\|u_{x}\right\|_{L^{2}}^{2}+\|u\|_{L^{2}}^{6}\right)+\frac{c_{0}}{3}\left\|u_{x}\right\|_{L^{2}}^{2}\|u\|_{L^{2}}^{4} \\
& =2 E(u)+\frac{d_{0}}{4}\|u\|_{L^{2}}^{6}+\left(\frac{d_{0}}{4}+\frac{c_{0}}{3}\|u\|_{L^{2}}^{4}\right)\left\|u_{x}\right\|_{L^{2}}^{2},
\end{aligned}
$$

logo pelo fato de que $E$ e $F$ são quantidades conservadas deduzimos que

$$
\left(1-h\left(\|\phi\|_{L^{2}}\right)\right)\left\|u_{x}\right\|_{L^{2}}^{2} \leq 2 E(\phi)+2 d_{0} F(\phi)^{3}
$$

$\operatorname{com} h(x)=\frac{d_{0}}{4}+\frac{c_{0}}{3} x^{4}$

Agora, seja $\phi$ satisfazendo a condição $1-h\left(\|\phi\|_{L^{2}}\right)>0$. Obtemos então que

$$
\left\|u_{x}(t)\right\|_{L^{2}}^{2} \leq \frac{2 E(\phi)+2 d_{0} F(\phi)^{3}}{1-h\left(\|\phi\|_{L^{2}}\right)} .
$$

Como queríamos provar.

Observação 4.1.3. Um resultado de boa colocação global para o problema de Cauchy (4.2) no espaço de energia $H_{\text {per }}^{1}$ quando a não linearidade $G$ tem a forma $G_{0}(u)=$ $u\left(\lambda|u|^{2}+\mu|u|^{4}\right)$ e $\mu \leq 0$, pode ser mostrado. Neste caso, não é necessario assumir que o dado inicial $\phi$ tenha norma $L_{\text {per }}^{2}$ pequena.

As observações 4.1.2 e 4.1.3 serão importantes no estudo da estabilidade de ondas viajantes associadas ao problema (4.2) quando a não linearidade $G$ tem a forma $G_{0}(u)=$ $u\left(\lambda|u|^{2}+\mu|u|^{4}\right)$, e os parametros $\lambda$ e $\mu$ são escolhidos de um modo conveniente. Ver capítulo 6.

Observação 4.1.4. Usando os resultados de boa colocação local obtidos nesta seção, assim como a teoria de estabilidade orbital que estabeleceremos ao longo deste trabalho para a CQ-S no caso periódico, é possivel obter um teorema de existência de 
soluções globais perto da órbita $\Omega_{a_{w}}$ (ver teorema 4.4.1). Este resultado, é essencial em nosso estudo de estabilidade.

\subsection{Existência de ondas periódicas para a equação CQ-S}

Nesta seção mostraremos como é possível obter a hipótese H-1 dada no capítulo anterior, isto é, mostraremos essencialmente a existência de uma curva suave de standing waves, ou seja,

$$
u(x, t)=e^{-i w t} a(x),
$$

onde $w \in \mathbb{R}$ e $a: \mathbb{R} \rightarrow \mathbb{R}$ é uma função periódica suave, $a(x+L)=a(x)$, para todo $x \in \mathbb{R}$. A ferramenta fundamental para este fim é a teoria das funções elípticas de Jacobi aasim como o Teorema da Função Implícita. Mostraremos também que quando o período $L$ tende ao infinito, as ondas periódicas tendem à onda solitária dadas pela fórmula (4.64) (ver Ohta [43]). Assim, substituindo (4.46) em (4.1), obtemos a seguinte equação diferencial ordinária não linear de segunda ordem,

$$
a^{\prime \prime}+w a+a^{3}+a^{5}=0,
$$

que pode ser escrita em forma de quadratura como,

$$
\left[a^{\prime}\right]^{2}=-w a^{2}-\frac{a^{4}}{2}-\frac{a^{6}}{3}+2 c
$$

onde $c$ é uma constante de integração. Agora fazendo, $b:=a^{2}$, a equação (4.48) pode ser reescrita em função de $b$ como

$$
\left[b^{\prime}\right]^{2}=-\frac{4}{3}\left[3 w b^{2}+\frac{3}{2} b^{3}+b^{4}-6 c b\right]=-\frac{4}{3} R(b),
$$

aqui $R$ é um polinômio com coeficientes reais definido por $R(t)=3 w t^{2}+\frac{3}{2} t^{3}+t^{4}-6 c t$. Com o objetivo de obter soluções explícitas da equação (4.49) vamos fazer algumas suposições sobre as raízes do polinômio $R$. Como zero é uma raiz de $R$, vamos assumir 
que as outras três raizes $\alpha_{1}, \alpha_{2}, \alpha_{3}$ são números reais e eles satisfazem $\alpha_{1}<0<\alpha_{2}<\alpha_{3}$. Logo $R$ pode ser fatorado como, $R(t)=t\left(t-\alpha_{1}\right)\left(t-\alpha_{2}\right)\left(t-\alpha_{3}\right)$, e assim, a equação (4.49) pode ser reescrita na forma

$$
\begin{aligned}
{\left[b^{\prime}\right]^{2} } & =-\frac{4}{3} b\left(3 w b+\frac{3}{2} b^{2}+b^{3}-6 c\right) \\
& =-\frac{4}{3} b\left(b-\alpha_{1}\right)\left(b-\alpha_{2}\right)\left(b-\alpha_{3}\right) .
\end{aligned}
$$

Notemos que a relação entre as raízes do polinômio $R$ e os seus coeficientes vem dada pelo seguinte sistema algébrico,

$$
\left\{\begin{aligned}
\alpha_{1}+\alpha_{2}+\alpha_{3} & =-\frac{3}{2} \\
\alpha_{1} \alpha_{2}+\alpha_{1} \alpha_{3}+\alpha_{2} \alpha_{3} & =3 w \\
\alpha_{1} \alpha_{2} \alpha_{3} & =6 c .
\end{aligned}\right.
$$

Como estamos procurando por $b$ positiva (pois $b=a^{2}$ ), então temos que $\alpha_{1}<0<$ $\alpha_{2}<b<\alpha_{3}$. Ainda mais, de (4.50) obtemos

$$
\int_{b}^{\alpha_{3}} \frac{d t}{\sqrt{t\left(\alpha_{3}-t\right)\left(t-\alpha_{2}\right)\left(t-\alpha_{1}\right)}}=\frac{2}{\sqrt{3}}\left(\xi+M_{0}\right)
$$

onde $M_{0}$, é uma constante de integração. Agora, usando Byrd \& Friedman [15] (fórmula 257.00) obtemos que a integral (4.52) pode ser invertida como,

$$
b(\xi)=\frac{\alpha_{3}\left(\alpha_{2}-\alpha_{1}\right)+\alpha_{1}\left(\alpha_{3}-\alpha_{2}\right) \operatorname{sn}^{2}\left(\frac{2}{\sqrt{3} g} \xi ; k\right)}{\left(\alpha_{2}-\alpha_{1}\right)+\left(\alpha_{3}-\alpha_{2}\right) \operatorname{sn}^{2}\left(\frac{2}{\sqrt{3} g} \xi ; k\right)},
$$

onde $g=\frac{2}{\sqrt{\alpha_{3}\left(\alpha_{2}-\alpha_{1}\right)}}$ e $k^{2}=\frac{-\alpha_{1}\left(\alpha_{3}-\alpha_{2}\right)}{\alpha_{3}\left(\alpha_{2}-\alpha_{1}\right)}$. Usando o fato que $1-k^{2} s n^{2}(x)=d n^{2}(x)$, temos que $b$ pode ser reescrita como

$$
a^{2}(\xi)=b(\xi)=\alpha_{3} \frac{d n^{2}\left(\frac{2 \xi}{\sqrt{3} g} ; k\right)}{1+\beta^{2} s n^{2}\left(\frac{2 \xi}{\sqrt{3} g} ; k\right)},
$$

onde $\beta^{2}=\frac{-\alpha_{3} k^{2}}{\alpha_{1}}>0$. Logo uma solução de (4.47) é dada por,

$$
a(\xi)=\sqrt{\alpha_{3} \frac{d n^{2}\left(\frac{2 \xi}{\sqrt{3} g} ; k\right)}{1+\beta^{2} \operatorname{sn}^{2}\left(\frac{2 \xi}{\sqrt{3} g} ; k\right)}}=\sqrt{\alpha_{3}} \frac{d n\left(\frac{2 \xi}{\sqrt{3} g} ; k\right)}{\sqrt{1+\beta^{2} s n^{2}\left(\frac{2 \xi}{\sqrt{3} g} ; k\right)}} .
$$

Notamos de (4.51) que $w<0$. Considerando $w=-4$ e $\alpha_{3}=2$, obtemos o seguinte gráfico para $a$, que tem como periódo minimal $T_{a} \approx 1.80487$ : 


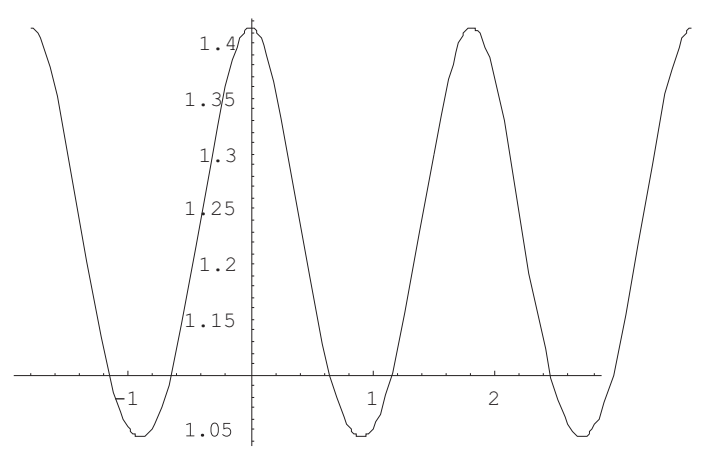

Agora apresentamos uma expressão para o período minimal de $a$ em (4.55). Como $d n$, $s n^{2}$ tém período minimal $2 K(k)$, então $a$ tem período minimal $L=\sqrt{3} g K(k)$, de onde deduzimos que a onda periódica $a$ tem período fundamental dado por

$$
T_{a}=\sqrt{3} g K(k)=\frac{2 \sqrt{3} K\left(k_{b}\right)}{\sqrt{\alpha_{3}\left(\alpha_{2}-\alpha_{1}\right)}},
$$

com $k^{2}=\frac{-\alpha_{1}\left(\alpha_{3}-\alpha_{2}\right)}{\alpha_{3}\left(\alpha_{2}-\alpha_{1}\right)}$. Á seguir estudaremos o período $T_{a}$. Se fixamos $w$ em (4.51), é possível mostrar que $\alpha_{2}, \alpha_{3}$ devem satisfazer a equação

$$
\alpha_{2}^{2}+\left(a_{3}+\frac{3}{2}\right) \alpha_{2}+\alpha_{3}^{2}+\frac{3}{2} \alpha_{3}+3 w=0
$$

e então, $\alpha_{1}, \alpha_{2}$ são dados por

$$
\alpha_{1}=\frac{-\left(\alpha_{3}+\frac{3}{2}\right)-\sqrt{m\left(\alpha_{3}\right)}}{2} \text { e } \alpha_{2}=\frac{-\left(\alpha_{3}+\frac{3}{2}\right)+\sqrt{m\left(\alpha_{3}\right)}}{2},
$$

com $m\left(\alpha_{3}\right)=-3 \alpha_{3}^{2}-3 \alpha_{3}-12 w+\frac{9}{4}$. Agora, devido às restrições impostas nas raízes de $R$, temos que $w$ e $c$ devem satisfazer,

$$
w<0, \text { e } c<0 \text {. }
$$

De (4.51), (4.58) e as condições impostas sobre $\alpha_{1}, \alpha_{2}, \alpha_{3}$, deduzimos que

$$
\alpha_{1}<0<\alpha_{2}<\frac{-1+\sqrt{1-4 w}}{2}<\alpha_{3}<\frac{-3+\sqrt{9-48 w}}{4} .
$$

Além disso, de (4.51) e (4.58) vemos que $T_{a}$ pode-se escrever na forma

$$
T_{a}\left(\alpha_{3}\right)=\frac{2 \sqrt{3} K\left(k\left(\alpha_{3}\right)\right)}{\sqrt{\alpha_{3}}\left(m\left(\alpha_{3}\right)\right)^{\frac{1}{4}}},
$$


com $k$ dado pela fórmula,

$$
k^{2}=\frac{3 \alpha_{3}^{2}+\frac{9}{2} \alpha_{3}+6 w+\alpha_{3} \sqrt{m\left(\alpha_{3}\right)}}{2 \alpha_{3} \sqrt{m\left(\alpha_{3}\right)}} .
$$

Esta última relação implica um interessante comportamento assintótico da solução (4.54). Mais especificamente, (4.54) e (4.62) mostram que se $\alpha_{3} \rightarrow \frac{-1+\sqrt{1-4 w}}{2}(w$ fixado), então $k \rightarrow 0$ e $b_{w}(\xi):=b(\xi) \rightarrow \frac{-1+\sqrt{1-4 w}}{2}$. Assim, nós obtemos a solução constante não trivial e positiva para (4.47),

$$
a \equiv \sqrt{\frac{-1+\sqrt{1-4 w}}{2}} .
$$

Da mesma forma, $\operatorname{como} d n(u, 1)=\operatorname{sech}(u), \operatorname{sn}(u, 1)=\tanh (u)$ e $\operatorname{sech}^{2}\left(\frac{u}{2}\right)=\frac{2}{\cosh (u)+1}$, temos que se $\alpha_{3} \rightarrow \frac{-3+\sqrt{9-48 w}}{4}$ então $k \rightarrow 1, \alpha_{2} \rightarrow 0$, e portanto

$$
a_{w}(\xi)=\left[\frac{1}{-4 w}+\frac{\sqrt{9-48 w}}{-12 w} \cosh (2 \sqrt{-w} \xi)\right]^{-\frac{1}{2}}
$$

que é a família de ondas solitárias positivas para (4.47) definidas para todo $w<0$. O gráfico da onda solitaria $a_{w}$ para $w<0$ é dada de uma forma geral por,

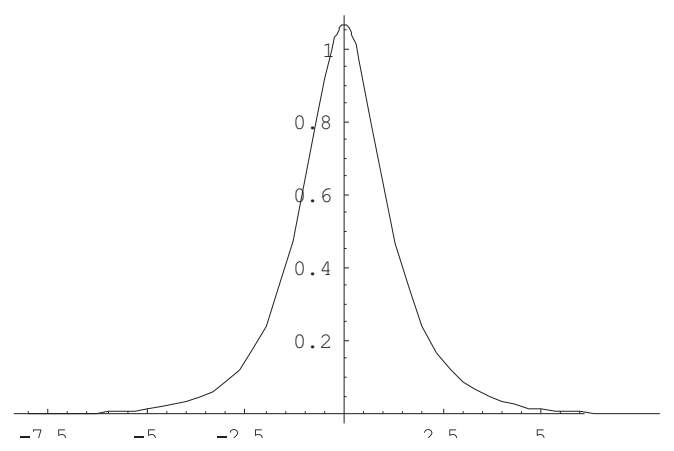

Agora, obtemos uma limitação inferior para $T_{a}$ a qual é fundamental na construção de uma curva suave de soluções a com um período minimal arbitrário. Observamos que a função $\alpha \in\left(\frac{-1+\sqrt{1-4 w}}{2}, \frac{-3+\sqrt{9-48 w}}{4}\right) \mapsto T_{a}(\alpha)$ satisfaz as seguintes relações, para $s=\frac{-1+\sqrt{1-4 w}}{2}$ e $v=\frac{-3+\sqrt{9-48 w}}{4}$ temos que:

$$
\begin{gathered}
\text { Se } \alpha \rightarrow s, \text { então } T_{a}(\alpha) \rightarrow \frac{\sqrt{3} \pi}{\sqrt{s}[m(s)]^{\frac{1}{4}}}=\frac{2 \pi}{\sqrt{1-4 w-\sqrt{1-4 w}}}=: \min (w), \\
\text { Se } \alpha \rightarrow v, \operatorname{então} T_{a}(\alpha) \rightarrow \infty \text { e assim }
\end{gathered}
$$

$$
T_{a}>\min (w) \text {. }
$$


A última afirmação se deduz do crescimento estrito da função $\alpha \rightarrow T_{a}(\alpha)$ (este resultado será mostrado no Teorema 4.2.1).

Observação 4.2.1. Notamos que, sempre que o parâmetro $w$ é grande em valor absoluto então a equação (4.47) tem soluções periódicas com período arbitrariamente pequeno $T_{a}$. Esta afirmação pode ser deduzida das relações (4.65), particularmente do fato que

$$
\text { se } w \rightarrow-\infty, \quad \text { ent } \tilde{a} o \quad \frac{2 \pi}{\sqrt{1-4 w-\sqrt{1-4 w}}} \rightarrow 0 \text {. }
$$

O gráfico seguinte mostra o comportamento da função período, para $w=-4 \mathrm{e}$ $1.56155<\alpha_{3}<2.79436$

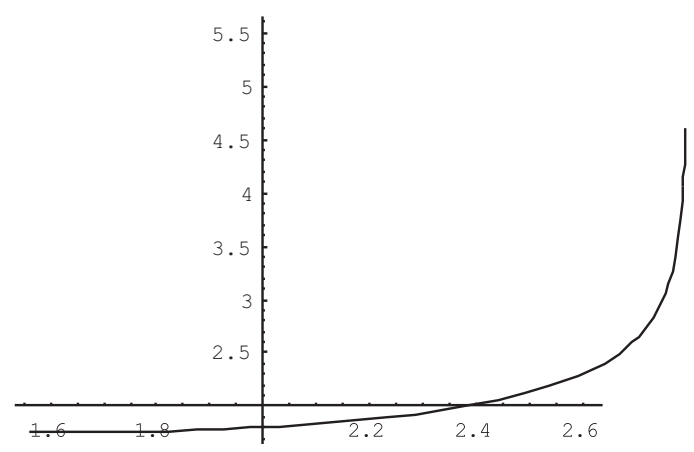

Agora, vamos construir uma função suave $w \rightarrow a_{w}$, de soluções periódicas positivas para a equação (4.47) com um período minimal fixado $L$. Da informação obtida acima, podemos construir uma solução positiva da equação (4.47) com perfil (4.55) e período $L$. De fato, seja $w<0$, tal que $L>\frac{\sqrt{3} \pi}{\sqrt{s}[m(s)]^{\frac{1}{4}}}$ (ver Observação(4.2.1)), então como a aplicação $\alpha \in\left(\frac{-1+\sqrt{1-4 w}}{2}, \frac{-3+\sqrt{9-48 w}}{4}\right) \mapsto T_{a}(\alpha)$ é estritamente crescente (ver Teorema 4.2.1), existe um único $\alpha \equiv \alpha_{w} \in\left(\frac{-1+\sqrt{1-4 w}}{2}, \frac{-3+\sqrt{9-48 w}}{4}\right)$ tal que $T_{a}\left(\alpha_{w}\right)=L$. Logo, de (4.58) e (4.62) conseguimos uma solução explícita para (4.47) na forma (4.55) e dependendo só de $\alpha=\alpha_{3}$. O seguinte teorema mostra como a função $w \rightarrow a_{w}$ é suave.

Teorema 4.2.1. Seja $L>0$ arbitrário embora fixado. Considere $w_{0}<0$ com $L>$ $\frac{2 \pi}{\sqrt{1-4 w_{0}-\sqrt{1-4 w_{0}}}}$ e um único $\alpha_{3,0}=\alpha\left(w_{0}\right) \in\left(\frac{-1+\sqrt{1-4 w_{0}}}{2}, \frac{-3+\sqrt{9-48 w_{0}}}{4}\right)$ tal que $T_{a}\left(\alpha_{3,0}\right)=$ $L$, então 
(1) Existe um intervalo aberto $J_{w_{o}} \subset \mathbb{R}$ contendo o ponto $w_{0}$, e um intervalo $I_{\alpha_{3,0}}$ contendo $\alpha_{3,0}$ e uma única função suave $\Gamma: J_{w_{o}} \rightarrow I_{\alpha_{3,0}}$ tal que $\Gamma\left(w_{0}\right)=\alpha_{3,0}$ e

$$
T_{a}\left(\alpha_{3}\right)=\frac{2 \sqrt{3} K(k)}{\sqrt{\alpha_{3}}\left(-3 \alpha_{3}^{2}-3 \alpha_{3}-12 w+\frac{9}{4}\right)^{\frac{1}{4}}}=L,
$$

com $\alpha_{3}=\alpha_{3}(w)=\Gamma(w)$ e $k^{2}=k^{2}(w)$ é dado por (4.62).

(2) A solução periódica $a_{w}\left(\cdot, \alpha_{3}\right)=\sqrt{b_{w}(\cdot)}$, determinada por $\alpha_{3}$, tem período fundamental $L$ e satisfaz (4.47). Além disso, a aplicação $w \in J_{w_{o}} \mapsto a_{w} \in H_{p e r}^{1}([0, L])$ é uma função suave.

(3) O intervalo $J_{w_{o}}$ nos items anteriores pode ser escolhido como $J_{w_{o}}=(-\infty, f(L))$, onde

$$
f(L)=\frac{1-\left(\frac{1+\sqrt{1+\frac{16 \pi^{2}}{L^{2}}}}{2}\right)^{2}}{4}<0 .
$$

Demonstração. Consideremos o conjunto aberto $\Omega \subset \mathbb{R}^{2}$, definido por

$$
\Omega=\left\{(\alpha, w) \mid w<0, \text { e } \alpha \in\left(\frac{-1+\sqrt{1-4 w}}{2}, \frac{-3+\sqrt{9-48 w}}{4}\right)\right\} .
$$

Definindo $F: \Omega \rightarrow \mathbb{R}$ como,

$$
F(\alpha, w)=\frac{2 \sqrt{3} K(k(\alpha, w))}{\sqrt{\alpha}\left(-3 \alpha^{2}-3 \alpha-12 w+\frac{9}{4}\right)^{\frac{1}{4}}},
$$

onde $k^{2}(\alpha, w)$ é dado em (4.62). Queremos mostrar que $F_{\alpha}(\alpha, w)>0$. Calculando $F_{\alpha}$, temos que

$$
F_{\alpha}(\alpha, w)=\frac{2 \sqrt{3}}{\left(\alpha^{2} m(\alpha)\right)^{\frac{5}{4}}}\left[\alpha^{2} m(\alpha) \frac{d K}{d k} \frac{d k}{d \alpha}-\frac{3}{4} \alpha K(k) s(\alpha)\right]
$$

onde $s(\alpha):=-4 \alpha^{2}-3 \alpha-8 w+\frac{3}{2}$ e $m(\alpha)=-3 \alpha^{2}-3 \alpha-12 w+\frac{9}{4}$. Por outro lado, de (4.62) deduzimos que

$$
\frac{d k}{d \alpha}=\frac{9 \alpha}{8 k\left(\alpha^{2} m(\alpha)\right)^{\frac{3}{2}}}[r(\alpha)]
$$

$\operatorname{com} r(\alpha)=2 \alpha^{3}+3 \alpha^{2}+6 w \alpha+w(16 w-3)$. Agora, se $\alpha>\frac{-1+\sqrt{1-4 w}}{2}$ então o polinômio $r(\alpha)>0$, e assim obtemos que $\frac{d k}{d \alpha}>0$. 
Agora, usando (4.68) e (4.69) obtemos as seguintes identidades,

$$
\begin{gathered}
\frac{\left(\alpha^{2} m(\alpha)\right)^{\frac{5}{4}}}{3 \sqrt{3} \alpha} F_{\alpha}= \\
\frac{1}{k} \frac{d K}{d k} \frac{3 r(\alpha)}{4\left(\alpha^{2} m(\alpha)\right)^{\frac{1}{2}}}-\frac{1}{2}\left(-4 \alpha^{2}-3 \alpha-8 w+\frac{3}{2}\right) K(k)= \\
\frac{1}{\left(\alpha^{2} m(\alpha)\right)^{\frac{1}{2}}}\left[\frac{1}{k} \frac{d K}{d k} \frac{3 r(\alpha)}{4}-\frac{1}{2}\left(\alpha^{2} m(\alpha)\right)^{\frac{1}{2}}\left(-4 \alpha^{2}-3 \alpha-8 w+\frac{3}{2}\right) K(k)\right]= \\
\frac{3 r(\alpha)}{4\left(\alpha^{2} m(\alpha)\right)^{\frac{1}{2}}}\left[\frac{1}{k} \frac{d K}{d k}-\frac{2}{3} \frac{\left(\alpha^{2} m(\alpha)\right)^{\frac{1}{2}}\left(-4 \alpha^{2}-3 \alpha-8 w+\frac{3}{2}\right) K(k)}{r(\alpha)}\right]> \\
\frac{3 r(\alpha)}{4\left(\alpha^{2} m(\alpha)\right)^{\frac{1}{2}}}\left[\frac{1}{k} \frac{d K}{d k}-K\right] .
\end{gathered}
$$

Como $\frac{1}{k} \frac{d K}{d k}-K>0$, então $F_{\alpha}(\alpha, w)>0$. Assim, do Teorema da Função Implícita obtemos uma função suave $\Gamma$ definida numa vizinhança $B_{w_{o}}$ de $w_{o}$, tal que $F(\Gamma(w), w)=$ $L$ para todo $w \in J_{w_{o}}$. Isto mostra os items (1) e (2).

O item (3) é uma consequência do comportamento da função $w \in(-\infty, 0) \rightarrow$ $\min (w)=\frac{2 \pi}{\sqrt{1-4 w-\sqrt{1-4 w}}}(\operatorname{ver}(4.65))$. De fato, é possível mostrar que a função min é estritamente crescente em $(-\infty, 0)$ e $\min (f(L))=L$. Isto mostra o teorema.

As fórmulas (4.71), (4.72), (4.74) e (4.76) abaixo, serão importantes no cálculo numérico da convexidade da função $d$ definida em (3.13). O seguinte corolario é deduzido do Teorema 4.2.1:

Corolário 4.2.1. A função $\Gamma(w)=\alpha_{3}(w)$, do Teorema 4.2.1 decresce.

Demonstração. De fato temos a seguinte fórmula para $\frac{d \alpha_{3}}{d w}$,

$$
\frac{d \alpha_{3}}{d w}=-\frac{F_{w}}{F_{\alpha_{3}}}=\frac{-\alpha_{3}\left[m\left(\alpha_{3}\right) \frac{d K}{d k} \frac{d k}{d w}+3 K(k)\right]}{\left[\alpha_{3} m\left(\alpha_{3}\right) \frac{d K}{d k} \frac{d k}{d \alpha_{3}}+\frac{3}{4} K(k)\left(4 \alpha_{3}^{2}+3 \alpha_{3}+8 w-\frac{3}{2}\right)\right]},
$$

do Teorema 4.2.1, temos que $F_{\alpha_{3}}\left(\alpha_{3}, w\right)>0$. Como,

$$
\frac{d k}{d w}=\frac{9 \alpha_{3}^{2}}{4 k\left[\alpha_{3}^{2} m\left(\alpha_{3}\right)\right]^{\frac{3}{2}}}\left[\alpha_{3}-4 w+\frac{3}{2}\right]>0
$$

$K(k)>0, \frac{d K}{d k}>0 \forall k \in(0,1)$, e $m\left(\alpha_{3}\right)>0$, então,

$$
\frac{d \alpha_{3}}{d w}<0,
$$


como queríamos provar.

Usando a fórmula (4.71) e as expressões explícitas para $\alpha_{1}, \alpha_{2}$, dadas em (4.58), é possível obter expressões para $\frac{d \alpha_{1}}{d w}$ e $\frac{d \alpha_{2}}{d w}$.

\section{Corolário 4.2.2.}

$$
\begin{aligned}
2 \frac{d \alpha_{1}}{d w} & =\left[\frac{6 \alpha_{3}+3}{2 m\left(\alpha_{3}\right)^{\frac{1}{2}}}-1\right] \frac{d \alpha_{3}}{d w}+\frac{6}{m\left(\alpha_{3}\right)^{\frac{1}{2}}}, \\
2 \frac{d \alpha_{2}}{d w} & =\left[-\frac{6 \alpha_{3}+3}{2 m\left(\alpha_{3}\right)^{\frac{1}{2}}}-1\right] \frac{d \alpha_{3}}{d w}-\frac{6}{m\left(\alpha_{3}\right)^{\frac{1}{2}}} .
\end{aligned}
$$

Demonstração. Imediata.

Agora, de (4.61) e Teorema 4.2.1 obtemos que

$$
\alpha_{3}\left[m\left(\alpha_{3}\right)\right]^{\frac{1}{2}}=\frac{12 K^{2}}{L^{2}}
$$

substituindo esta expressão em (4.62) então é possível obter a seguinte fórmula para $w$ em função de $\alpha_{3}$

$$
w=-\frac{1}{2} \alpha_{3}^{2}-\frac{3}{4} \alpha_{3}+\frac{2 K(k)^{2}\left(2 k^{2}-1\right)}{L^{2}} .
$$

Agora, de (4.73) temos que $\alpha_{3}^{2} m\left(\alpha_{3}\right)=\frac{144 K^{4}}{L^{4}}$ e como $m\left(\alpha_{3}\right)=-3 \alpha_{3}^{2}-3 \alpha_{3}-12 w+\frac{9}{4}$, deduzimos que

$$
\alpha_{3}^{4}+\alpha_{3}^{3}+\left(4 w-\frac{3}{4}\right) \alpha_{3}^{2}+\frac{48 K^{4}}{L^{4}}=0
$$

de onde substituindo (4.74) em (4.75) obtemos a seguinte equação polinômial para $\alpha_{3}$,

$$
\alpha_{3}^{4}+2 \alpha_{3}^{3}+\left[\frac{3}{4}-\frac{8 K(k)^{2}\left(2 k^{2}-1\right)}{L^{2}}\right] \alpha_{3}^{2}-\frac{48 K(k)^{4}}{L^{4}}=0,
$$

para $L$ fixado. A equações (4.76) e (4.74) nos dão a possibilidade de expressar $\alpha_{3} \mathrm{e}$ $w$ em função da variável $k$, ditas expressões serão importantes no cálculo numérico da convexidade da função $d$ em (3.13).

As seguintes duas seções serão dedicadas a mostrar as hipoteses H-2 e H-3 dadas no capítulo 3 e que irão implicar o Teorema 3.1.1 para o caso em que $a$ tem a forma (4.55). 


\subsection{Teoria espectral para a equação CQ-S}

Nesta seção mostraremos como obter as hipóteses H-2, H-3 discutidas no capítulo anterior. Naturalmente estas informações serão parte importante na prova do Teorema 3.1.1. Iniciamos considerando o espaço complexo $X=H_{\text {per }}^{1}([0, L])$, o qual será dotado do produto interno real

$$
(u, v)=\operatorname{Re} \int_{0}^{L} u_{x} \bar{v}_{x}+u \bar{v} d x
$$

e denotaremos por $\langle$,$\rangle o parênteses de dualidade entre X$ e $X^{*}$,

$$
\langle f, u\rangle=\operatorname{Re} \int_{0}^{L} f \bar{u} d x
$$

Mostraremos que a hipótese H-2 é satisfeita para a curva $w \rightarrow a_{w} \in H_{p e r}^{1}([0, L])$ determinada na seção 4.2. Para isto, lembramos que os funcionais $E, F: X \rightarrow \mathbb{R}$, definidos em (3.6a) e (3.6b) são suaves. Escreveremos a avaliação de suas primeiras derivadas como $\left\langle E^{\prime}(u), v\right\rangle$ e $\left\langle F^{\prime}(u), v\right\rangle$ respectivamente, onde $E^{\prime}, F^{\prime}: X \rightarrow X^{*}$, e as suas segundas derivadas como $\left\langle E^{\prime \prime}(u) w, v\right\rangle$ e $\left\langle F^{\prime \prime}(u) w, v\right\rangle$, para $w \in X$. Assim, para $u \in X$, temos que

$$
\left\{\begin{array}{l}
E^{\prime}(u)=-u_{x x}-|u|^{2} u-|u|^{4} u, \quad \mathrm{e} \\
F^{\prime}(u)=u .
\end{array}\right.
$$

Logo, segue-se imediatamente que $a_{w}$ é um ponto crítico do funcional $\Psi=E-w F$, isto é de (3.4),

$$
\begin{aligned}
\Psi^{\prime}\left(a_{w}\right) & =E^{\prime}\left(a_{w}\right)-w F^{\prime}\left(a_{w}\right) \\
& =-\left(a_{w}^{\prime \prime}+a_{w}^{3}+a_{w}^{5}+w a_{w}\right)=0 .
\end{aligned}
$$

A seguir vamos deduzir formalmente os operadores $\mathcal{L}_{R}$ e $\mathcal{L}_{I m}$ definidos em (3.12). Para isto, consideremos inicialmente $u, \phi \in X$. Logo de (4.79) temos que

$$
\begin{aligned}
& E^{\prime \prime}(u) \phi=-\phi_{x x}-\left(2|u|^{2}+3|u|^{4}\right) \phi-\left(u^{2}+2|u|^{2} u^{2}\right) \bar{\phi} \mathrm{e} \\
& F^{\prime \prime}(u) \phi=\phi .
\end{aligned}
$$

Para $\phi=z_{1}+i z_{2}$, onde $z_{1}=\operatorname{Re}(\phi)$ e $z_{2}=\operatorname{Im}(\phi)$, temos que $\Psi^{\prime \prime}\left(a_{w}\right) \phi$ é dado pelo operador linear,

$$
\Psi^{\prime \prime}\left(a_{w}\right) \phi=\mathcal{L}_{R}\left(z_{1}\right)+i\left[\mathcal{L}_{I m}\left(z_{2}\right)\right]
$$


onde $\mathcal{L}_{R}, \mathcal{L}_{\text {Im }}$ resultam ser os operadores de tipo Hill dados por,

$$
\begin{aligned}
\mathcal{L}_{R} & =-\frac{d^{2}}{d x^{2}}-w-3 a^{2}-5 a^{4}, \quad \mathrm{e} \\
\mathcal{L}_{\text {Im }} & =-\frac{d^{2}}{d x^{2}}-w-a^{2}-a^{4} .
\end{aligned}
$$

Aqui $a=a_{w}$ é definida em (4.55). O estudo espectral dos operadores lineares em (4.83) será o ponto chave em nossa teoria de estabilidade. Devido à forma específica do potencial $Q(x)=-w-3 a^{2}(x)-5 a^{4}(x)$ em (4.55) a teoria clássica de Floquet para operadores de tipo Hill nos fornece a informação requerida nas hipóteses H-3.1 e H-3.2 para $\mathcal{L}_{R}$. Assim uma opção será aplicar a teoria estabelecida no Teorema 3.2.3 acima.

Como veremos a teoria de Floquet será sim importante no estudo do operador $\mathcal{L}_{\text {Im }}$. Antes de continuar nosso estudo dos operadores $\mathcal{L}_{R}$ e $\mathcal{L}_{\text {Im }}$, observamos que da fórmula (3.7) (simetrias da CQ-S) é possível deduzir que o operador $\Psi^{\prime \prime}\left(a_{w}\right)$ satisfaz as seguintes duas propriedades básicas,

$$
\begin{aligned}
& \Psi^{\prime \prime}\left(a_{w}\right) T_{1}^{\prime}(0) a_{w}=0 \quad \mathrm{e} \\
& \Psi^{\prime \prime}\left(a_{w}\right) T_{2}^{\prime}(0) a_{w}=0 .
\end{aligned}
$$

Como,

$$
\begin{aligned}
& T_{1}^{\prime}(0) a_{w}=-i a_{w} \quad \mathrm{e} \\
& T_{2}^{\prime}(0) a_{w}=-a_{w}^{\prime}
\end{aligned}
$$

obtemos de (4.82), (4.84) que,

$$
\mathcal{L}_{R} a_{w}^{\prime}=0 \quad \text { e } \quad \mathcal{L}_{I m} a_{w}=0
$$

O seguinte teorema resume as propriedades espectrais básicas para o operador $\mathcal{L}_{\text {Im }}$.

Teorema 4.3.1. (Propriedades Espectrais de $\mathcal{L}_{\text {Im }}$ ). O operador $\mathcal{L}_{\text {Im }}$ definido em (4.83) é um operador fechado e auto-adjunto. Seu espectro é só composto por uma sequência limitada inferiormente de números reais $\left\{\mu_{i}\right\}_{i=0}^{\infty}$ satisfazendo

$$
\mu_{0}=0<\mu_{1} \leqslant \mu_{2} \cdots
$$

$e \lim _{i \rightarrow \infty} \mu_{i}=\infty$. Em particular, zero é um autovalor simples com autofunção associada $a$. 
Demonstração. A primeira parte do teorema segue do Teorema 3.2.1. Por outro lado como $a_{w}(x)>0 \forall x \in \mathbb{R}$ e $\mathcal{L}_{I m} a(x)=0, \forall x \in[0, L]$, temos pelo Teorema de Oscilação para equações de Hill (ver [39]), que zero deve ser o primeiro autovalor para o operador $\mathcal{L}_{\text {Im }}$. Logo zero é simples e temos a distribuição de autovalores para $\mathcal{L}_{\text {Im }}$ tal como estabelecida no teorema. Isto finaliza a prova.

As propriedades espectrais que temos para o operador $\mathcal{L}_{R}$ são resumidas no seguinte teorema.

Teorema 4.3.2. (Propriedades Espectrais de $\mathcal{L}_{R}$ ). O operador $\mathcal{L}_{R}$ definido em (4.83) é um operador fechado e auto-adjunto. Seu espectro é composto de uma sequência de autovalores reais $\left\{\lambda_{i}\right\}_{i=0}^{\infty}$ limitados inferiormente satisfazendo, $\lambda_{0} \leq \lambda_{1} \leq \lambda_{2} \cdots$, e $\lim _{i \rightarrow \infty} \lambda_{i}=\infty$. Em particular o primeiro autovalor $\lambda_{0}$ de $\mathcal{L}_{R}$ é negativo e simples. Além disso, o autovalor $\lambda_{1}$ é zero e também é simples com autofunção $\frac{d}{d x} a$. O resto do espectro esta distante de zero. Assim, nos temos a seguinte distribuição dos autovalores $\operatorname{para} \mathcal{L}_{R}$,

$$
\lambda_{0}<0<\lambda_{2}<\lambda_{3} \leq \lambda_{4}<\cdots
$$

Demonstração. A prova segue das idéias apresentadas em [44]. Assim, do Teorema 3.2.1 com $M=-\frac{d^{2}}{d x^{2}}$ e $f(x)=x^{3}+x^{5}$, segue que o espectro do operador $\mathcal{L}_{R}$ é composto de uma sequência de autovalores reais $\left\{\lambda_{i}\right\}_{i=0}^{\infty}$ limitados inferiormente satisfazendo,

$$
\lambda_{0} \leq \lambda_{1} \leq \lambda_{2}
$$

e $\lim _{i \rightarrow \infty} \lambda_{i}=\infty$. Agora, para provar que $\lambda_{0}$ é um autovalor simples e que zero é o segundo autovalor simples associado com o operador $\mathcal{L}_{R}$ vamos aplicar o Teorema 3.2.3. Isto é, devemos mostrar que:

(i) $\widehat{a}(k)>0, k \in \mathbb{Z}$, é uma sequência par e positiva.

(ii) A transformada de Fourier de $f^{\prime}(a)=3 a^{2}+5 a^{4}=5 a^{2}\left(\frac{3}{5}+a^{2}\right)$ está na classe $P F(2)$ discreto.

Assim, lembramos inicialmente que $a$ representa uma solução par periódica e positiva de (4.47), com um período minimal fixado $L$. Dedicaremos primeiro a nossa 
atenção em provar o item (ii) acima. Para isto, é necessario primeiro calcular a série de Fourier de $a^{2}=b$ em (4.54). Começamos lembrando a definição de integral elíptica de terceiro tipo $\Lambda$, a saber,

$$
\Lambda(u, \alpha, k)=\int_{0}^{u} \frac{d x}{1-\alpha^{2} s n^{2}(x ; k)},
$$

onde $k$ é o modulo elíptico e $\alpha$ pode ser visto como sendo,

$$
\begin{aligned}
& 0<\alpha^{2}<k^{2}<1, \text { ou }, 0<k^{2}<\alpha^{2}<1 \text { ou }, \\
& \alpha^{2}>1 \text { ou } \alpha^{2}=-\beta^{2} \text { e } \beta>0 .
\end{aligned}
$$

De (4.54) tomamos $\alpha^{2}=-\beta^{2}=\alpha_{3} k^{2} / \alpha_{1}<0$ e assim da primeira equação em (4.51), $0<-\alpha^{2}<k^{2}$. Por tanto de [15] (fórmula 431.04), segue que

$$
\int_{0}^{u_{1}} \frac{d n^{2}(x)}{1-\alpha^{2} s n^{2}(x)} d x=C(\alpha, k)\left[u_{1} \Lambda_{0}(p ; k)+\Omega_{1}\left(u_{1}\right)\right]
$$

$\operatorname{com} p=\operatorname{sen}^{-1} \sqrt{\frac{\alpha^{2}}{\alpha^{2}-k^{2}}}=\operatorname{sen}^{-1} \frac{\beta}{\sqrt{\beta^{2}+k^{2}}}, \Lambda_{0}(p ; k)$ é a função Lambda de Heuman (ver Definição (4.90) abaixo), e

$$
C(\alpha, k)=\frac{\pi\left(k^{2}-\alpha^{2}\right)}{2 \sqrt{\alpha^{2}\left(1-\alpha^{2}\right)\left(\alpha^{2}-k^{2}\right)} K(k)}, \quad \Omega_{1}\left(u_{1}\right)=\frac{i K}{\pi} \ln \frac{\vartheta_{0}(\varrho-i v)}{\vartheta_{0}(\varrho+i v)},
$$

onde $\varrho=\varrho\left(u_{1}\right)=\frac{L u_{1}}{2 K}, v=\frac{L F\left(p ; k^{\prime}\right)}{2 K}$. Aqui $\vartheta_{0}$ é a função Theta (ver capítulo 2 ) e $F(\cdot ; \cdot)$ é a integral elíptica normal de primeiro tipo (ver capítulo 2).

Se consideramos $u_{1}=\frac{1}{2 g} \xi$, obtemos da regra de Leibniz aplicada a (4.88) que $b$ em (4.54) pode ser escrita como

$$
a^{2}(\xi)=b(\xi)=C\left(\alpha_{3}, \alpha, k\right)\left[\Lambda_{0}(p ; k)+\frac{i}{2 \pi}\left(\frac{\vartheta_{0}^{\prime}(\xi-i v)}{\vartheta_{0}(\xi-i v)}-\frac{\vartheta_{0}^{\prime}(\xi+i v)}{\vartheta_{0}(\xi+i v)}\right)\right]
$$

onde $C\left(\alpha_{3}, \alpha, k\right) \equiv \alpha_{3} C(\alpha, k)$ e $\Lambda_{0}(p ; k)$ é definido como,

$$
\begin{aligned}
\Lambda_{0}(p ; k) & =\frac{2}{\pi}\left[K(k) E\left(p ; k^{\prime}\right)-K(k) F\left(p ; k^{\prime}\right)+E(k) F\left(p ; k^{\prime}\right)\right] \\
& =\frac{F\left(p ; k^{\prime}\right)}{K\left(k^{\prime}\right)}+\frac{2}{\pi} Z\left(p ; k^{\prime}\right) K(k) .
\end{aligned}
$$

Aqui $Z(\cdot, \cdot)$ representa a função Zeta de Jacobi (ver capítulo 2). Agora, de [15] (fórmula 1050.02), deduzimos as seguintes representações,

$$
\frac{\vartheta_{0}^{\prime}(\xi-i v)}{\vartheta_{0}(\xi-i v)}=4 \pi \sum_{n=1}^{\infty} a_{n} \operatorname{sen}\left(\frac{2 \pi n}{L}(\xi-i v)\right)=
$$




$$
4 \pi \sum_{n=1}^{\infty} a_{n}\left[\operatorname{sen}\left(\frac{2 \pi n \xi}{L}\right) \cos \left(\frac{2 \pi n i v}{L}\right)-\operatorname{sen}\left(\frac{2 \pi n i v}{L}\right) \cos \left(\frac{2 \pi n \xi}{L}\right)\right],
$$

onde $a_{n}=\frac{1}{2} \operatorname{csch}\left(\frac{n \pi K^{\prime}}{K}\right), K^{\prime}(k) \equiv K\left(k^{\prime}\right)\left(k^{\prime}=\sqrt{1-k^{2}}\right)$, e

$$
\begin{gathered}
\frac{\vartheta_{0}^{\prime}(\xi+i v)}{\vartheta_{0}(\xi+i v)}= \\
4 \pi \sum_{n=1}^{\infty} a_{n}\left[\operatorname{sen}\left(\frac{2 \pi n \xi}{L}\right) \cos \left(\frac{2 \pi n i v}{L}\right)+\operatorname{sen}\left(\frac{2 \pi n i v}{L}\right) \cos \left(\frac{2 \pi n \xi}{L}\right)\right] .
\end{gathered}
$$

Logo de (4.89) temos finalmente a representação em serie de Fourier de $b$,

$$
b(\xi)=C\left(\alpha_{3}, \alpha, k\right)\left[\Lambda_{0}(p ; k)+2 \sum_{n=1}^{\infty} \frac{\operatorname{senh}\left(\frac{\pi F\left(p, k^{\prime}\right) n}{K}\right)}{\operatorname{senh}\left(\frac{\pi K^{\prime} n}{K}\right)} \cos \left(\frac{2 \pi n \xi}{L}\right)\right] .
$$

Agora verificamos que a sequência $\{\widehat{b}(n)\}_{n \in \mathbb{Z}}$, está em $P F(2)$ discreto. Inicialmente encontramos uma função $s: \mathbb{R} \rightarrow \mathbb{R}$ tal que $s \in P F(2)$-continuo (ver capítulo 2) e tal que $s(n)=\widehat{b}(n)$ para $n \in \mathbb{Z}$. Para isto, vamos tomar os coeficientes da série de Fourier da função $b$ calculada em (4.91). De fato, como

$$
q(x)=\frac{\operatorname{senh}(\nu x)}{\operatorname{senh}(\mu x)}
$$

é logaritmicamente côncava para $0<\nu<\mu$, ou seja, $\frac{d^{2}}{d x^{2}} \log (q(x))<0$ para $x \neq 0$, obtemos que $q$ está na classe $P F(2)$ contínuo (ver Lema 2.0.1 no capítulo 2). Agora, como $p \in\left[0, \frac{\pi}{2}\right]$ implica $F\left(p ; k^{\prime}\right) \in\left[0, K^{\prime}\right]$, deduzimos que para todo $k \in(0,1)$ fixado, a sequência

$$
\left(a_{n}\right)_{n \in \mathbb{Z}}=\left(\frac{\operatorname{senh}\left(\frac{n \pi F\left(p ; k^{\prime}\right)}{K}\right)}{\operatorname{senh}\left(\frac{n \pi K^{\prime}}{K}\right)}\right)_{n \in \mathbb{Z}}
$$

pertence a $P F(2)$ no caso discreto.

Como

$$
a_{0}=\frac{F\left(p ; k^{\prime}\right)}{K^{\prime}}<\Lambda_{0}(p ; k)
$$

pois,

$$
\Lambda_{0}(p ; k)=\frac{F\left(p ; k^{\prime}\right)}{K^{\prime}(k)}+\frac{2}{\pi} K(k) Z\left(p ; k^{\prime}\right)>\frac{F\left(p ; k^{\prime}\right)}{K^{\prime}(k)}
$$

é possível redefinir a seguinte função contínua de classe $P F(2), \tau: \mathbb{R} \rightarrow \mathbb{R}$

$$
\tau(x)=\alpha_{3} C(\alpha, k) \frac{\operatorname{senh}\left(\frac{\pi F\left(p ; k^{\prime}\right) x}{K}\right)}{\operatorname{senh}\left(\frac{\pi K^{\prime} x}{K}\right)},
$$


por uma função diferencíavel $s: \mathbb{R} \rightarrow \mathbb{R}$ tal que $s(0)=\alpha_{3} C(\alpha, k) \Lambda_{0}(p ; k), s(x)=\tau(x)$ em $(-\infty, 1] \cup[1, \infty)$, e definida em $(-1,1)$ tal que $s \in P F(2)$ no caso continuo. Portanto concluimos que, $(\widehat{b}(n))_{n \in \mathbb{Z}}=\left(\widehat{a^{2}}(n)\right)_{n \in \mathbb{Z}}$ pertence a $P F(2)$-discreto. Assim, via um análise como acima, e usando que

$$
\widehat{\frac{3}{5}+a^{2}}(n)=\left\{\begin{array}{l}
\widehat{a^{2}}(n) \quad \text { se } n \neq 0 \\
\frac{3}{5}+\widehat{a^{2}}(0) \quad \text { se } n=0
\end{array}\right.
$$

obtemos que a sequência $\left(\widehat{\frac{3}{5}+a^{2}}(n)\right)_{n \in \mathbb{Z}}$ está na classe $P F(2)$-discreto. Agora do Teorema 3.2.2 concluímos que

$$
\left(3 a^{2}+5 a^{4}\right)^{\wedge}(n)=5 \widehat{a^{2}} *\left(\widehat{\frac{3}{5}+a^{2}}\right)(n)
$$

está na classe $P F(2)$-discreto. Assim, provamos o item (ii) acima.

Procedemos agora a mostrar o item (i) acima. Isto é, devemos mostrar que $\widehat{a}(k)>0$ para todo $k \in \mathbb{Z}$. Primeiramente notemos que a função $\frac{a^{2}}{\alpha_{3}}$ em (4.54) pode ser escrita como

$$
\frac{a^{2}}{\alpha_{3}}=\frac{1}{1+\left(k^{2}+\beta^{2}\right) s d^{2}\left(\frac{2 \xi}{\sqrt{3} g} ; k\right)},
$$

onde $s d(x ; k):=\frac{s n(x ; k)}{d n(x ; k)}$. Agora, definindo a função $h$ como $h(x):=\frac{a(x)}{\sqrt{\alpha_{3}}}$ deduzimos de (4.93) que $0<h \leq 1$. Portanto temos que $0<h^{2} \leq h \leq 1$ e $\widehat{h}(0)=\frac{1}{L} \int_{0}^{L} h(x) d x>0$. Agora, vamos supor por absurdo que existe um $n_{0} \in \mathbb{N}$ com $\widehat{a}\left(n_{0}\right) \leq 0$. Claramente $\widehat{h}\left(n_{0}\right) \leq 0$. Consideremos uma função a valores reais $f$ suficientemente regular tal que $f>0$,

$$
\widehat{f}(n)= \begin{cases}\widehat{f}(0) & \text { se } n=0, \\ \widehat{f}\left(n_{0}\right) & \text { se } n=n_{0}, \\ 0 & \text { em outro caso. }\end{cases}
$$

Com $\widehat{f}(0)>0$ e satisfazendo a relação

$$
\widehat{f}\left(n_{0}\right) \widehat{h^{2}}\left(n_{0}\right)>\widehat{f}(0) \widehat{h}(0)+\widehat{f}\left(n_{0}\right) \widehat{h}\left(n_{0}\right),
$$


então do fato que $\widehat{h}\left(n_{0}\right) \leq 0$ é possível mostrar que $\widehat{f}\left(n_{0}\right)>0$. Logo a relação (4.95) pode ser escrita como

$$
0<\frac{\widehat{f}(0)}{\widehat{f}\left(n_{0}\right)}<\frac{\widehat{h^{2}}\left(n_{0}\right)-\widehat{h}\left(n_{0}\right)}{\widehat{h}(0)} .
$$

Vamos mostrar que dita função $f$ existe. Com efeito, seja $\left\{r_{n}\right\}_{n \in \mathbb{Z}}$ uma sequência tal que $r_{0}>0, r_{n_{0}}>0$ e $r_{n}=0$ para todo $n \neq 0, n_{0}$. É claro que $\left\{r_{n}\right\}_{n \in \mathbb{Z}} \in \mathcal{S}(\mathbb{Z})$

(ver capítulo 2), assim existe uma função suave $f \in \mathcal{P}$ tal que $\widehat{f}(n)=r_{n}$ para todo $n \in \mathbb{Z}$. Pelo Teorema de Fourier (ver Teorema 14 de [31]) podemos escolher $f>0$. A relação (4.95) pode ser obtida fazendo uma escolha conveniente dos coeficientes $r_{0} \mathrm{e}$ $r_{n_{0}}$. Agora, usando a positividade da função $f$, a identidade de Perseval e que $h \geq h^{2}$ obtemos,

$$
\begin{aligned}
\widehat{f}(0) \widehat{h}(0)+\widehat{f}\left(n_{0}\right) \widehat{h}\left(n_{0}\right) & =\int_{0}^{L} f(x) h(x) d x \\
& \geq \int_{0}^{L} f(x) h^{2}(x) d x=\widehat{f}(0) \widehat{h^{2}}(0)+\widehat{f}\left(n_{0}\right) \widehat{h^{2}}\left(n_{0}\right),
\end{aligned}
$$

como $\widehat{f}(0) \widehat{h^{2}}(0)>0$, deduzimos de $(4.97)$ que $\widehat{f}(0) \widehat{h}(0)+\widehat{f}\left(n_{0}\right) \widehat{h}\left(n_{0}\right)>\widehat{f}\left(n_{0}\right) \widehat{h^{2}}\left(n_{0}\right)$. Isto contradiz (4.95). Isto finaliza a prova do Teorema 4.3.2.

\subsection{Condição de convexidade da função $d$}

Nesta seção vamos mostrar a convexidade da função $d$ (ver Teorema 3.1.1). Como veremos abaixo um cálculo explícito desta torna-se muito complicado pela natureza das funções implicadas. Nós só conseguimos obter esta propriedade através do uso de métodos numéricos. Seja então $f(L)$ como no Teorema 4.2 .1 e $d:(-\infty, f(L)) \rightarrow \mathbb{R}$, definida por

$$
d(w)=\Psi\left(a_{w}\right)=E\left(a_{w}\right)-w F\left(a_{w}\right)
$$

com $E, F$, dadas em (3.6). Da hipótese H-2 no Capítulo 3 vemos que

$$
d^{\prime}(w)=-F\left(a_{w}\right)
$$


A segunda derivada da função $d$, vem dada por

$$
d^{\prime \prime}(w)=-\frac{d}{d w} F\left(a_{w}\right)=-\frac{d}{d w} \int_{0}^{L} a_{w}^{2}(x) d x .
$$

Agora, avaliaremos $\left\langle a_{w}, a_{w}\right\rangle$, de fato, usando o Teorema 4.2.1

$$
\begin{aligned}
\left\langle a_{w}, a_{w}\right\rangle & =\int_{0}^{L} a_{w}^{2}(\xi) d \xi \\
& =\int_{0}^{L} \alpha_{3} \frac{d n^{2}\left(\frac{\xi}{2 g} ; k\right)}{1+\beta^{2} s n^{2}\left(\frac{\xi}{2 g} ; k\right)} d \xi \\
& =4 \alpha_{3} g \int_{0}^{K} \frac{d n^{2}(r ; k)}{1+\beta^{2} s n^{2}(r ; k)} d r .
\end{aligned}
$$

Logo, usando a fórmula (410.04) em [15], temos que

$$
\left\langle a_{w}, a_{w}\right\rangle=2 \pi \alpha_{3} g \frac{k^{2}-\alpha^{2}}{\sqrt{\alpha^{2}\left(1-\alpha^{2}\right)\left(\alpha^{2}-k^{2}\right)}} \Lambda_{0}(p ; k),
$$

onde $\alpha^{2}=-\beta^{2}=\frac{\alpha_{3} k^{2}}{\alpha_{1}}, p=\operatorname{sen}^{-1}\left(\sqrt{\frac{\alpha^{2}}{\alpha^{2}-k^{2}}}\right)=\operatorname{sen}^{-1}\left(\frac{\beta}{\sqrt{\beta^{2}+k^{2}}}\right), \sqrt{3} g=\frac{L}{K}$ e $\Lambda_{0}$ definida em (4.90). É possível reescrever $\left\langle a_{w}, a_{w}\right\rangle$ em (4.102) na forma,

$$
\begin{aligned}
& \left\langle a_{w}, a_{w}\right\rangle=\left[\frac{\alpha_{1} \alpha_{3}\left(\alpha_{1}-\alpha_{3}\right)}{\alpha_{3} k^{2}-\alpha_{1}}\right]^{\frac{1}{2}} Q(p, k), \mathrm{com} \\
& Q(p, k)=\frac{L \pi}{2} \frac{\Lambda_{0}(p ; k)}{K(k)}
\end{aligned}
$$

Neste ponto, para obter uma expressão explícita para $\partial_{w}\left\langle a_{w}, a_{w}\right\rangle$ é importante notar que,

i) Pelo Teorema 4.2.1, o período $L$ das ondas periódicas $a_{w}$ definidas em (4.55) é considerado constante.

ii) As variáveis $\alpha_{1}, \alpha_{2}, \alpha_{3}, p, k$ que aparecem na fórmula (4.103) são na verdade funções da variável $w$, de fato o Teorema 4.2.1 assegura que $\alpha_{3}=\alpha_{3}(w)$, a fórmula (4.58) nos permite escrever $\alpha_{1}=\alpha_{1}(w)$ e $\alpha_{2}=\alpha_{2}(w)$. Por último as expressões (4.62) e (4.102) mostram que $k=k(w)$ e $p=p(w)$ respectivamente.

iii) De (4.62) e (4.72) é possível conseguir uma fórmula para calcular $\frac{d k}{d w}$ como função das variáveis $\alpha_{3}, w$ e $k$. Logo (do item ii)) só em função de $w$. 
iv) As fórmulas (4.76), (4.74)e (4.58) nos permitem escrever $\alpha_{3}, \alpha_{2}, \alpha_{1}$ e $w$ como função da variável $k$, portanto $-d^{\prime \prime}(w)=\partial_{w}\left\langle a_{w}, a_{w}\right\rangle$ pode ser vista também como uma função da variável $k$.

Como a expressão explícita para $\partial_{w}\left\langle a_{w}, a_{w}\right\rangle$ é muito longa e difícil de analisar analíticamente, nesta parte do trabalho vamos exibir o gráfico da função $-d^{\prime \prime}:(-\infty, f(L)) \subset \mathbb{R} \rightarrow \mathbb{R}$ usando o programa Mathematica. De fato, a fórmula (4.103), junto às expressões (4.71) (4.72) e (2.10), nos permitem obter uma fórmula para $d^{\prime \prime}$ que depende das variáveis $\alpha_{1}, \alpha_{2}, \alpha_{3}, w$ e $k$. Na verdade, do item iv) acima deduzimos que $d^{\prime \prime}$ pode ser vista como função da variável $k$. O seguinte gráfico nos mostra o comportamento de $-d^{\prime \prime}$, $\left(-d^{\prime \prime}=-d^{\prime \prime}(k, L)\right)$,

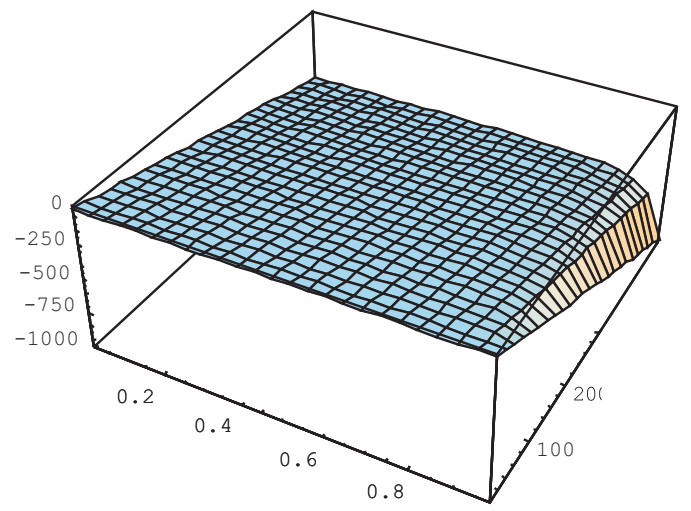

para $0<k<1,0<L<500$, será sempre negativo. Se fizermos por exemplo $L=300$, então temos o seguinte gráfico para $-d^{\prime \prime}$

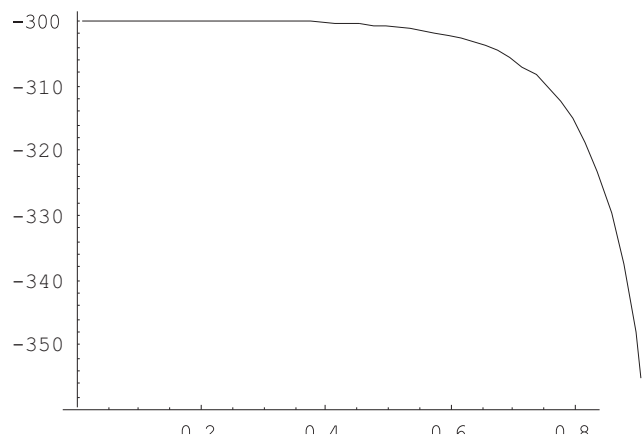

Isto finaliza o nosso estudo da convexidade da função $d$. 
Agora, devido a que não temos um resultado de boa colocação global em $H_{p e r}^{1}([0, L])$ para a Eq. CQ-S (ver seção 4.1). O resultado de estabilidade orbital para a órbita $\Omega_{a_{w}}$ no Teorema 3.1.1, é apenas valido para os valores de $t$ onde a solução $u(t)$ existe. Porém, dito resultado junto com o teorema de boa colocação local para a CQ-S (ver Teorema 4.1.3), implicam a existência global de soluções quando o dado inicial está perto da órbita. Assim, temos o seguinte teorema:

Teorema 4.4.1. (Existência global de soluções próximas da órbita) Toda solução u da equação $C Q-S$, com dado inicial $u(0)=f$ próximo da órbita $\Omega_{a_{w}}$, é globalmente bem definida.

Demonstração. Obviamente, para todo $s \in \mathbb{R}$ temos

$$
\begin{aligned}
\|u(t)\|_{1} & \leq\left\|u(t)-e^{-i s} a_{w}(\cdot-y)\right\|_{1}+\left\|e^{-i s} a_{w}(\cdot-y)\right\|_{1} \\
& =\left\|u(t)-e^{-i s} a_{w}(\cdot-y)\right\|_{1}+\left\|a_{w}\right\|_{1} .
\end{aligned}
$$

Assim, de (4.104) e da estabilidade da órbita $\Omega_{a_{w}}$, temos que, para $\epsilon>0$ existe $\delta>0$ tal que

$$
\|u(t)\|_{1} \leq \epsilon+\left\|a_{w}\right\|_{1}, \quad \forall t \in\left[0, T^{*}(f)\right),
$$

sempre que $\inf _{s \in[0,2 \pi), y \in[0, L)}\left\|f-e^{-i s} a_{w}(\cdot-y)\right\|_{1}<\delta$, aqui $T^{*}(f)$ denota o tempo maximal de existência da solução $u$ dado pelo Teorema 4.1.3.

Em particular, (4.105) implica a limitação da solução $u$. Isto junto com o Teorema 4.1.4 prova o teorema.

Assim, do Teorema 4.4.1 e dos resultados obtidos nas seções 4.2, 4.3 e 4.4, temos como consequência o Teorema 3.1.1 (ver apêndice A).

\subsection{Instabilidade por perturbações subharmônicas}

O Teorema 3.1.1 estabelece a estabilidade orbital de standing waves do tipo $u(x, t)=$ $e^{-i w t} a_{w}(x)$, no espaço $H_{p e r}^{1}([0, L])$ para a equação,

$$
i u_{t}+u_{x x}+u\left(|u|^{2}+|u|^{4}\right)=0
$$


com $L$ denotando o período minimal de $a_{w}$ (ver (4.55). Agora, como o perfil da onda $a_{w} \in H_{p e r}^{1}([0, j L])$ com $j \in \mathbb{N}, j>1$, então é natural se perguntar pela estabilidadeinstabilidade da órbita gerada pela onda $e^{-i w t} a_{w}$ no caso de ter perturbações no espaço $H_{p e r}^{1}[0, j L]$. Como veremos abaixo, de fato esta resulta ser linearmente instável. Como consequência disto e usando a teoria em Grillakis [30], concluímos que esta órbita de fato é instável do ponto de vista não linear (Teorema 4.5.3 abaixo).

Começamos nossa análise com o seguinte resultado da teoria de Floquet.

Lema 4.5.1. Definimos $P_{j}$ e $Q_{j}$ como sendo o número de autovalores negativos de $\mathcal{L}_{R}$ $e \mathcal{L}_{\text {Im }}$, respectivamente, com condições de fronteira periódicas em $[0, j L], j \geq 2$. Então $Q_{j}=0$ e $P_{j}=2 j$ ou $P_{j}=2 j-1$.

Demonstração. Como $a_{w}(x)>0$ e $\mathcal{L}_{\text {Im }} a(x)=0, \forall x \in[0, j L]$, temos pelo Teorema de Oscilação de Sturm-Liouville para equações de Hill, que zero deve ser o primeiro autovalor e portanto para todo $j \geq 2, Q_{j}=0$. Por outro lado, como $\mathcal{L}_{R} a^{\prime}=0$, para $x \in[0, j L]$, e o número de zeros da função $a^{\prime}$ no intervalo aberto $[0, j L)$ é $2 j$, então de novo o Teorema de Oscilação implica que o autovalor correspondente ao autovalor zero é $\lambda_{2 j}$ ou $\lambda_{2 j-1}$. De onde obtemos que $P_{j}=2 j$ ou $P_{j}=2 j-1$. Isto finaliza a prova do lema.

Uma base teórica para provar instabilidade não-linear de um resultado de instabilidade linear para equações de tipo Schrödinger foi desenvolvido por Grillakis-Jones (1988). Desde esse ponto de vista, é possível obter instabilidade linear quando o "número de autovalores negativos de $\mathcal{L}_{R}$ excede o número de autovalores negativos de $\mathcal{L}_{\text {Im }}$ por mais de um "(ver Teoremas 4.5.1, 4.5.2 abaixo). As condições de instabilidade em Grillakis [28] que permitem obter a instabilidade linear de $a=a_{w}$, conectam $P_{j}, Q_{j}$ e a existência de autovalores reais positivos do operador matricial,

$$
N_{j}=\left[\begin{array}{cc}
0 & \mathcal{L}_{I m} \\
-\mathcal{L}_{R} & 0
\end{array}\right]
$$

A seguir daremos as definições necéssarias para estabelecer os resultados em Grillakis (1988) 
1. Seja $K_{j}$ a projeção ortogonal sobre $\left(\operatorname{Ker} \mathcal{L}_{R}\right)^{\perp}$.

2. Seja $R_{j}$ o operador $R_{j} \equiv K_{j} \mathcal{L}_{R} K_{j}$.

3. Seja $S_{j}$ o número de autovalores negativos de $R_{j}$.

4. Seja $I_{\text {real }}\left(N_{j}\right)$, o número de pares de autovalores reais não nulos de $N_{j}$, visto sobre $[0, j L]$.

Teorema 4.5.1. [Grillakis (1988)], Para $j \geq 1$, obtemos,

1- Se $\left|S_{j}-Q_{j}\right| \equiv m_{j}>0$, então $I_{\text {real }}\left(N_{j}\right) \geq m_{j}$.

2- Se $S_{j}=Q_{j}$ e $\left\{f \in L_{\text {per }}^{2}([0, j L]) \mid\left(R_{j} f, f\right)<0 \quad e \quad\left(\left(\mathcal{L}_{I m}\right)^{-1} f, f\right)<0\right\}=\emptyset$, então $I_{\text {real }}\left(N_{j}\right) \geq 1$.

O seguinte resultado dá uma condição para calcular $S_{j}$.

Teorema 4.5.2. [Grillakis (1988)] $S e-\frac{d}{d w} \int_{0}^{j L} a_{w}^{2}(x) d x>0$, então $S_{j}=P_{j}-1$.

O seguinte Teorema é o resultado principal desta seção.

Teorema 4.5.3. [Instabilidade não linear] Consideramos a onda $a_{w}$ dada pelo Teorema 4.2.1. Então a órbita $\Omega_{a_{w}}=\left\{e^{i s} a_{w} \mid s \in \mathbb{R}\right\}$ é instável em $H_{p e r}^{1}([0, j L])$, para todo $j \geq 2$ pelo fluxo da CQ-S.

Demonstração. Será mostrado de fato que a órbita $\Omega_{a_{w}}$ é linearmente instável. Para este fim, vamos reescrever a equação (4.106) na forma Hamiltoniana

$$
\frac{d \mathbf{u}}{d t}=J E^{\prime}(\mathbf{u}(t))
$$

onde $\mathbf{u}=[\operatorname{Re}(u), \operatorname{Im}(u)]^{t}, J$ é a matriz anti-simétrica injetora e sobrejetora dada por

$$
J=\left[\begin{array}{cc}
0 & 1 \\
-1 & 0
\end{array}\right],
$$


e $E$ denotando o funcional linear definido em (3.6). Agora, para a linearização de (4.108) ao redor da órbita $\Omega_{a_{w}}$, escrevemos $\mathbf{A}_{w}(x)=\left[a_{w}(x), 0\right]^{t}$, e definimos

$$
\mathbf{v}(t)=T(w t) \mathbf{u}(t)-\mathbf{A}_{w},
$$

com,

$$
T(s)=\left[\begin{array}{cc}
\cos (s) & -\sin (s) \\
\sin (s) & \cos (s)
\end{array}\right],
$$

a matriz de rotação por um ângulo $s$. Agora de (4.110), deduzimos que

$$
\begin{aligned}
& \mathbf{u}^{\prime}(t)=T(-w t)\left[\mathbf{v}^{\prime}(t)+w J \mathbf{v}(t)+\mathbf{A}_{w}\right] \\
& J E^{\prime}(\mathbf{u}(t))=J E^{\prime}\left(T(-w t)\left[\mathbf{v}(t)+\mathbf{A}_{w}\right]\right) .
\end{aligned}
$$

Como $E^{\prime}(T(s) \mathbf{u})=T(s) E^{\prime}(\mathbf{u})$ para todo $s \in \mathbb{R}$, temos de (4.108) e (4.112) que

$$
\begin{aligned}
\mathbf{v}^{\prime}(t) & =J\left[E^{\prime}\left(\mathbf{v}(t)+\mathbf{A}_{w}\right)-w\left(\mathbf{v}(t)+\mathbf{A}_{w}\right)\right] \\
& =J\left[E^{\prime}\left(\mathbf{v}(t)+\mathbf{A}_{w}\right)-w\left(F^{\prime}\left(\mathbf{v}(t)+\mathbf{A}_{w}\right)\right)\right] \\
& =J\left[E^{\prime}\left(\mathbf{A}_{w}\right)-w F^{\prime}\left(\mathbf{A}_{w}\right)+E^{\prime \prime}\left(\mathbf{A}_{w}\right) \mathbf{v}(t)-w F^{\prime \prime}\left(\mathbf{A}_{w}\right) \mathbf{v}(t)+O\left(\|v\|^{2}\right)\right] \\
& =J\left[\left(E^{\prime \prime}\left(\mathbf{A}_{w}\right)-w F^{\prime \prime}\left(\mathbf{A}_{w}\right)\right) \mathbf{v}(t)\right]+O\left(\|v\|^{2}\right)=N \mathbf{v}(t)+O\left(\|v\|^{2}\right)
\end{aligned}
$$

nesta cadeia de igualdades foi usado o fato que $F^{\prime}(\mathbf{u})=\mathbf{u}$, o Teorema de Taylor, que $\mathbf{A}_{w}$ é um ponto critico do funcional $E-w F$ e a limitação do operador $J$. Aqui $N$ é o operador linear definido em (4.107).

Assim, em nosso estudo de instabilidade estamos interessados na existência de modos de crescimento para a equação linear (ou problema linearizado)

$$
\frac{d \mathbf{v}}{d t}=N(\mathbf{v}(t))
$$

Ou seja, queremos achar soluções para (4.114) na forma $\mathbf{v}(t)=e^{\lambda t} g(x) \operatorname{com} \operatorname{Re}(\lambda)>$ 0 e $g \in D(N)=H_{p e r}^{2}([0, L]) \times H_{p e r}^{2}([0, L])$. A seguir observamos inicialmente que os autovalores de $N$ aparecem em pares conjugados. Como foi visto na seção 4.4, $-\frac{d}{d w} \int_{0}^{L} a_{w}^{2}(x) d x>0$, assim que do Lema 4.5.1, Teorema 4.5.1 e Teorema 4.5.2 obtemos que $m_{j}=2 j-1$ ou $m_{j}=2 j-2$. Assim, para $j \geq 2$, temos que $I_{\text {real }}\left(N_{j}\right) \geq m_{j}$. Portanto 
obtemos a existência de modos de crescimento e isto implica que a solução zero de (4.114) é instável. Finalmente, seguindo os resultados em Grillakis\&Shatah\&Strauss [30], temos que a órbita $\Omega_{a_{w}}$ é instável em $H_{p e r}^{1}[0, j L]$.

\subsection{Estabilidade de soluções constantes}

Nesta seção mostraremos a estabilidade de standing waves do tipo $e^{-i w t} a_{w}$ associadas com a CQ-S quando o perfil $a_{w}$ é constante, não-trivial e positivo. Assim, da equação (4.47) é claro que $a_{w}$ deve satisfazer a seguinte equação algébrica

$$
a^{\prime \prime}+w a+a^{3}+a^{5}=w a+a^{3}+a^{5}=0 .
$$

De (4.115) facilmente deduzimos que $a \equiv a_{w}$ é dado por

$$
a_{w} \equiv \sqrt{\frac{-1+\sqrt{1-4 w}}{2}},
$$

para $w<0$ (ver fórmula (4.63)). A seguir, consideramos os operadores $\mathcal{L}_{R}$ e $\mathcal{L}_{\text {Im }}$ dados por

$$
\mathcal{L}_{R}=-\frac{d^{2}}{d x^{2}}-r(w), \quad \text { e } \quad \mathcal{L}_{I m}=-\frac{d^{2}}{d x^{2}},
$$

onde a constante $r(w)$ é dada por $r(w):=1-4 w-\sqrt{1-4 w}>0$. É claro que o operador $\mathcal{L}_{I m}$ é não negativo com zero como primeiro autovalor simples. Por outro lado o espectro $\sigma\left(\mathcal{L}_{R}\right)$, associado com o operador $\mathcal{L}_{R}$ é dado por

$$
\sigma\left(\mathcal{L}_{R}\right)=\left\{\frac{4 \pi^{2} k^{2}}{L^{2}}-r(w) \mid k=0,1,2,3, \ldots\right\}
$$

Portanto, tomando $w \in(f(L), 0)$ onde

$$
f(L):=\frac{1-\left(\frac{1+\sqrt{1+\frac{16 \pi^{2}}{L^{2}}}}{2}\right)^{2}}{4}<0,
$$

(ver Teorema 4.2.1) é possível mostrar que $-r(w)$ é o único autovalor negativo e simples do operador $\mathcal{L}_{R}$ com autofunção 1, o resto dos autovalores são estritamente positivos e tem como autoespaços de dimensão dois os conjuntos $\left\{\cos \left(\frac{2 \pi k x}{L}\right), \operatorname{sen}\left(\frac{2 \pi k x}{L}\right)\right\}$ para 
$k=1,2,3, \ldots$

Por outro lado, observamos que a condição de convexidade neste caso é trivialmente satisfeita. De fato temos que

$$
-\frac{d}{d w} \int_{0}^{L} a_{w}^{2} d x=\frac{L}{\sqrt{1-4 w}}>0 .
$$

Assim, estamos em condições de enunciar o seguinte resultado:

Teorema 4.6.1. Sejam $L>0$ e $w_{0}<0$ dados. Considere $\psi_{w_{0}} \equiv \sqrt{\frac{-1+\sqrt{1-4 w_{0}}}{2}} a$ solução constante positiva e não nula da equação (4.115). Então $\psi_{w_{0}}$ é estável em $H_{\text {per }}^{1}([0, L])$ pelo fluxo da $C Q-S$ desde que $w_{0}>f(L)$.

Nota: A seguir, comentaremos de forma rápida como é possível aplicar os resultados de existência e estabilidade orbital de standing waves obtidos nas seções 4.2, 4.3 e 4.4 para a equação CQ-S na equação:

$$
u_{t}+u_{x x x}+3 u^{2} u_{x}+5 u^{4} u_{x}=0
$$

que chamaremos de Modificada-Crítica $\mathrm{KdV}(\mathrm{MC}-\mathrm{KdV})$. Aqui $u: \mathbb{R} \times \mathbb{R} \rightarrow \mathbb{R}$ e $(x, t) \in \mathbb{R} \times \mathbb{R}$.

Como a equação (4.121) possui apenas presenta a simetria de translação, o tipo de onda viajante procurada neste caso tem a forma

$$
u(x, t)=a(x+w t):=T_{2}(-w t) a(x),
$$

$w \in \mathbb{R}$ e $a: \mathbb{R} \rightarrow \mathbb{R}$ denota uma função periódica suave. Substituindo (4.122) em (4.121), obtemos que $a$ satisfaz

$$
a^{\prime \prime}+w a+a^{3}+a^{5}=A,
$$

assim, considerando $A=0$ obtemos a equação (4.47). Desta forma, todos os resultados obtidos (nas seções anteriores) para as standing waves da CQ-S, continuam sendo válidos para as ondas viajantes periódicas do tipo (4.122). Em particular temos uma curva suave $w \rightarrow a_{w}$ com período fixado $L$ e perfil $a=a_{w}$ dado por (4.55).

A seguir, introduzimos os aspectos fundamentais na prova do Teorema 4.6.2 abaixo. 
1- Os funcionais $E, F: H_{p e r}^{1} \rightarrow \mathbb{R}$ definidos como

$$
E(u)=\frac{1}{2} \int_{0}^{L} u_{x}^{2}-\frac{u^{4}}{2}-\frac{u^{6}}{3} d x, \quad e \quad F(u)=\frac{1}{2} \int_{0}^{L} u^{2} d x
$$

são quantidades conservadas pelo fluxo da MC-KdV. Além disso E, F são quantidades invariantes sob o grupo $T_{2}$.

2- A onda viajante $a_{w}$ é um ponto crítico do funcional $\Psi=E-w F$ (ver hipótese H-2 no capítulo 3), isto é

$$
\Psi^{\prime}\left(a_{w}\right)=a^{\prime \prime}+w a+a^{3}+a^{5}=0
$$

3- A segunda variação associada ao funcional $\Psi$ neste caso é só dada por um operador:

$$
\mathcal{L}_{0}:=\Psi^{\prime \prime}\left(a_{w}\right)=-\frac{d^{2}}{d x^{2}}-w-3 a_{w}^{2}-5 a_{w}^{4}
$$

Comparando o operador $\mathcal{L}_{0}$ em (4.125) com o operador $\mathcal{L}_{R}$ dado em (4.83) deduzimos que as propriedades espectrais para $\mathcal{L}_{0}$ (ver hipótese H-3 no capítulo 3 ) continuam sendo válidas.

4- O análise da convexidade da função $d:(-\infty, f(L)) \rightarrow \mathbb{R}$ definida por $d(w)=\Psi\left(a_{w}\right)$, é a mesma mostrada para a função $d$ associada a equação CQ-S. (Ver seção 4.4).

Agora, se denotamos por $\Omega_{a_{w}}$ a órbita gerada por $a_{w}$, isto é, $\Omega_{a_{w}}=\left\{T_{2}(s) a_{w}(\cdot): s \in \mathbb{R}\right\}$, e para $\eta>0$ definamos $U_{\eta} \subset H_{\text {per }}^{1}$ por,

$$
F \in U_{\eta} \Leftrightarrow \rho\left(F, \Omega_{a_{w}}\right)=\inf _{g \in \Omega_{a_{w}}}\|F-g\|_{1}<\eta
$$

então temos o seguinte teorema sobre a estabilidade das ondas viajantes periódicas $a_{w}$ associadas a MC-KdV:

Teorema 4.6.2. (Estabilidade Orbital para a $\boldsymbol{M C}-\boldsymbol{K} \boldsymbol{d} \boldsymbol{V})$ A órbita $\Omega_{a_{w}}$ gerada por $a_{w}$, é estável pelo fluxo periódico da equação MC-KdV. Mais exatamente, dado $\epsilon>0$, existe $\delta(\epsilon)>0$ tal que para todo $u_{0} \in U_{\delta}$, a solução u associado a $M C-K d V$ com dado inicial $u(0)=u_{0}$, satisfaz $u(t) \in U_{\epsilon}$ para todo $t \in \mathbb{R}$. 


\section{CAPÍTULO 5}

\section{EQUAÇÃO DE SCHRÖDINGER CÚBICA-QUÍNTICA COM UMA INTERAÇÃO DE TIPO DELTA DE DIRAC (CQD-S)}

Neste capítulo, estamos interessados em estudar a existência e estabilidade orbital de standing waves

$$
u(x, t)=e^{-i w t} \phi_{w}(x)
$$

para a equação de Schrödinger cúbica-quíntica acoplada com uma Delta de Dirac (CQD-S), isto é,

$$
i u_{t}+u_{x x}+Z \delta(x) u+u\left(|u|^{2}+|u|^{4}\right)=0, \quad Z \in \mathbb{R}
$$

onde $\delta$ é a medida de Dirac centrada no ponto $x=0, u: \mathbb{R} \times \mathbb{R} \rightarrow \mathbb{C}$.

Na seção 5.1 daremos os resultados já conhecidos sobre existência e estabilidade em $H^{1}(\mathbb{R})$ de ondas viajantes para a CQ-S $(Z=0$ na equação (5.2)). Na seção 5.2 apresentaremos algumas propriedades espectrais do operador $-\frac{d^{2}}{d x^{2}}+\gamma \delta$ em uma dimensão. As soluções explícitas do tipo (5.1) serão estudadas na seção 5.4. O problema de estabilidade orbital das ondas dadas em (5.1) será abordado na seção 5.5. Em particular, estudaremos as propriedades espectrais dos operadores $\mathcal{L}_{1, Z}$ e $\mathcal{L}_{2, Z}$ dados em (5.49). A convexidade da função $w \rightarrow\left\|\phi_{w, Z}\right\|^{2}$ será estudada de maneira numérica 
na subseção 5.5.2. Finalmente, na subseção 5.5.3 daremos os resultados de estabilidadeinstabilidade associados com a equação CQD-S.

Antes de abordar o problema (5.2) de maneira geral, vamos primeiro a estabelecer algumas idéias de como obter a estabilidade orbital de standing waves no espaço $H^{1}(\mathbb{R})$ no caso em que $Z=0$ na equação (5.2). Embora este problema tinha sido estudado e resolvido por Ohta em [43], algumas informações deste análise serão usados em nosso estudo de estabilidade orbital para a Eq. (5.2). Em particular as propriedades espectrais do operador $\mathcal{L}_{1,0}$ dado em (5.3) junto a teoria de perturbação analítica de operadores não limitados nos permitirá deduzir informações essenciais sobre o espectro dos operadores $\mathcal{L}_{1, Z}, \mathcal{L}_{2, Z}$ dados em (5.49), vital em nosso análise de estabilidade.

\subsection{Estabilidade de standing waves para a equação CQ-S no caso da reta}

Nesta seção fazemos um resumo da estabilidade de standing waves do tipo (5.1) para a CQ-S $\left(Z=0\right.$ em (5.2)) no espaço $H^{1}(\mathbb{R})$. Em [43] o problema de estabilidade de standing waves deste tipo foi estudado usando como critério fundamental o estudo das propriedades espectrais dos operadores

$$
\begin{aligned}
& \mathcal{L}_{1,0}=-\frac{d^{2}}{d x^{2}}-w-3 \phi_{w}^{2}-5 \phi_{w}^{4}, \\
& \mathcal{L}_{2,0}=-\frac{d^{2}}{d x^{2}}-w-\phi_{w}^{2}-\phi_{w}^{4},
\end{aligned}
$$

onde $\phi_{w}$ é uma onda estacionaria do tipo solitária que será determinada abaixo. Assim como o estudo do crescimento ou decrescimento da função

$$
I(w)=-\int \phi_{w}^{2}(x) d x
$$

Neste caso, $\phi_{w}$ é a única solução positiva do problema elíptico não linear

$$
\left\{\begin{array}{l}
\phi^{\prime \prime}+w \phi+\phi^{3}+\phi^{5}=0 \\
\phi \in H^{1}(\mathbb{R}) .
\end{array}\right.
$$


Como vimos no capítulo anterior esta solução é dada pela fórmula, para $w<0$, por

$$
\phi_{w}(x)=\left[\frac{1}{-4 w}+\frac{\sqrt{9-48 w}}{-12 w} \cosh (2 \sqrt{-w} x)\right]^{-\frac{1}{2}} .
$$

Os seguintes teoremas dão uma descrição precisa sobre o espectro dos operadores $\mathcal{L}_{1,0}$, $\mathcal{L}_{2,0}$ em (5.3), definidos em $L^{2}(\mathbb{R})$. Suas provas são baseadas básicamente em que $\phi_{w}$ é positiva, $\phi_{w}^{\prime}(x)=0$ em $x=0$ e a teoria de Oscilação de Sturm-Liouville (ver [8]).

Teorema 5.1.1. Seja $w<0$. O operador $\mathcal{L}_{1,0}$ tem exatamente um único autovalor negativo simples $\tau_{0}$, o segundo autovalor é zero o qual tambem é simples com autofunção associada $\frac{d}{d x} \phi_{w}$. O resto do espectro é de tipo essencial e esta longe de zero, mais exatamente $\sigma_{\text {ess }}\left(\mathcal{L}_{1,0}\right)=[-w, \infty)$.

Teorema 5.1.2. Seja $w<0$. O operador $\mathcal{L}_{2,0}$ tem zero como único autovalor com autofunção associada $\phi_{w}$. O resto do espectro é de tipo essencial e é dado por $\sigma_{\text {ess }}\left(\mathcal{L}_{2,0}\right)=$ $[-w, \infty)$.

Agora, vamos calcular a quantidade $I(w)=-\int_{-\infty}^{\infty} \phi_{w}^{2}(x) d x$ usando principalmente o comportamento assintótico das soluções periódicas positivas associadas a equação (5.5), determinadas no Capítulo 4. Consideremos $w<0$ fixado e $a_{w, \alpha_{3}}$ a solução periódica dada em (4.55) satisfazendo a equação (5.5). Dos cálculos feitos no capítulo 4 Seção 4.2 , temos que se $\alpha_{3} \rightarrow \frac{-3+\sqrt{9-48 w}}{4}$, então

$$
\alpha_{1} \rightarrow \frac{-3-\sqrt{9-48 w}}{4}, \quad \alpha_{2} \rightarrow 0, \quad k \rightarrow 1
$$

Ainda mais

$$
a_{w, \alpha_{3}} \rightarrow \phi_{w, 0}
$$

uniformemente sobre intervalos fechados e limitados de $\mathbb{R}$. Agora, como $\sqrt{3} g=\frac{L}{K}$, $g=\frac{2}{\sqrt{\alpha_{3}\left(\alpha_{2}-\alpha_{1}\right)}}$ e $\Lambda_{0}(p, 1)=\frac{2}{\pi} p$, então das fórmulas (4.103) e (5.8), deduzimos que

$$
I(w)=-\int_{-\infty}^{\infty} \phi_{w}^{2}(x) d x=-2 \sqrt{3} \sin ^{-1}\left(\sqrt{\frac{1}{2}-\frac{3}{2 \sqrt{9-48 w}}}\right),
$$


$\log 0$

$$
-\frac{d}{d w}\left\|\phi_{w}\right\|^{2}=\frac{6}{(3-16 w) \sqrt{-w}}>0 .
$$

Então, seguindo as mesmas idéias usadas na prova de estabilidade das standing waves no caso periódico (ver Apêndice A) é possível mostrar o seguinte

Teorema 5.1.3. (Estabilidade Orbital para a $C Q-S$ no caso da reta) A órbita $\Omega_{\phi_{w}}$ gerada por $\phi_{w}$ em (5.6), é estável pelo fluxo da equação $C Q-S$ no espaço $H^{1}(\mathbb{R})$. Mais exatamente dado $\epsilon>0$, existe $\delta(\epsilon)>0$ tal que para todo $u_{0} \in U_{\delta}$, a solução u do problema de valor inicial associado a $C Q-S,(Z=0 \mathrm{em}(5.2)) \operatorname{com} u(0)=u_{0}$, satisfaz $u(t) \in U_{\epsilon}$ para todo $t \in \mathbb{R}$.

Observação 5.1.1. A boa colocação local em $H^{1}(\mathbb{R})$ do problema de Cauchy associado a equação (5.2) com $Z=0$, foi mostrada em [19]. As mesma idéias usadas para mostrar boa colocação local e global para a CQ-S no caso periódico (ver seção 4.1) continuam sendo válidas neste caso.

\section{$5.2 \delta$-interação em dimensão um.}

Nesta seção vamos entender o significado preciso da interação do tipo $\delta$ centrada no ponto $x=0$, determinada pela expresão formal

$$
-\frac{d^{2}}{d x^{2}}+\gamma \delta \equiv-\frac{d^{2}}{d x^{2}}+\gamma(\delta, \cdot) \delta
$$

onde o simbolo $\delta$ denota a função delta de Dirac, $(\delta, \psi)=\psi(0)$. $\gamma$ em (5.11) é denominada a constante de acoplamento. A maioria dos resultados nesta parte podem ser encontrados em [3].

Seja $A_{0}$ um operador simétrico densamente definido sobre um espaço de Hilbert, denotaremos por $A_{0}^{*}$ seu adjunto, consideramos os subespaços

$$
D_{+}=\operatorname{Ker}\left(A_{0}^{*}-i\right), \quad \text { e } \quad D_{-}=\operatorname{Ker}\left(A_{0}^{*}+i\right)
$$


$D_{+}$e $D_{-}$são chamados os subespaços de deficiência de $A_{0}$. O par de números $n_{+}\left(A_{0}\right), n_{-}\left(A_{0}\right)$, dados por

$$
n_{+}\left(A_{0}\right)=\operatorname{dim}\left[D_{+}\right], \quad \text { e } n_{-}\left(A_{0}\right)=\operatorname{dim}\left[D_{-}\right],
$$

são chamados os indices de deficiência do operador $A_{0}$. Agora consideremos o operador autoadjunto $A=-\frac{d^{2}}{d x^{2}}$ sobre $L^{2}(\mathbb{R})$ com seu domínio natural $D(A)=H^{2}(\mathbb{R})$. Então como $\delta \in H^{-1}(\mathbb{R})$ temos o seguinte resultado (ver [3]).

Lema 5.2.1. O operador de restrição $\left.A_{0} \equiv A\right|_{D\left(A_{0}\right)}$ com

$$
D\left(A_{0}\right)=\{g \in D(A) \mid \delta(g) \equiv g(0)=0\}
$$

é fechado, densamente definido e simétrico, com indices de deficiência iguais a 1. Precisamente temos

1- Fechado: $\overline{\Gamma\left(A_{0}\right)}=\Gamma\left(A_{0}\right)$, aqui $\Gamma\left(A_{0}\right)$ denota o gráfico do operador $A_{0}$.

2- simetria: $\left(A_{0} g, h\right)=\left(g, A_{0} h\right)$ para $g, h \in D\left(A_{0}\right)$.

3- densidade: $\overline{D\left(A_{0}\right)}=L^{2}(\mathbb{R})$.

4- Os elementos de deficiência de $A_{0}$ são,

$$
\left\{\begin{array}{l}
\text { Para } \lambda=i, \quad \psi_{i} \equiv(A-i)^{-1} \delta, \\
\text { Para } \lambda=-i, \quad \psi_{-i} \equiv(A+i)^{-1} \delta,
\end{array}\right.
$$

satisfazendo $\psi_{ \pm i} \in D\left(A_{0}^{*}\right)$ e $A_{0}^{*} \psi_{ \pm i}= \pm i \psi_{ \pm i}$.

Demonstração. A prova deste lema é analoga à prova presentada em [11], portanto aqui só mostraremos uma idéia da prova do item 4. Como $(A-i)^{-1} \in B\left(H^{-2}(\mathbb{R}) ; L^{2}(\mathbb{R})\right)$ então temos que $\psi_{i} \equiv(A-i)^{-1} \delta \in L^{2}(\mathbb{R})$ e como $\widehat{\delta}(\xi)=1$, entaõ para $g \in D\left(A^{0}\right) \subset$ $D(A)$, obtemos

$$
\begin{aligned}
\left\langle A^{0} g, \psi_{i}\right\rangle & =\left\langle A g,(A-i)^{-1} \delta\right\rangle=\int_{-\infty}^{\infty} \xi^{2} \widehat{g}(\xi) \overline{\frac{1}{\xi^{2}-i} \widehat{\delta}(\xi)} d \xi \\
& =g(0)+\int_{-\infty}^{\infty} \widehat{g}(\xi) \overline{i \widehat{\psi}_{i}(\xi)}=g(0)+\left\langle g, i \psi_{i}\right\rangle=\left\langle g, i \psi_{i}\right\rangle .
\end{aligned}
$$


Istos calculos implicam que $\psi_{i} \in D\left(A^{0^{*}}\right)$ e $A^{0^{*}}\left(\psi_{i}\right)=i \psi_{i}$. Da mesma forma é possível mostrar que $\psi_{-i} \in D\left(A^{0^{*}}\right)$ e $A^{0^{*}}\left(\psi_{-i}\right)=-i \psi_{-i}$.

Agora vamos a calcular explicitamente os elementos de deficiência $\psi_{ \pm i}$ do operador $A_{0}$. Observamos primeiro que a equação

$$
A_{0}^{*} \psi(k)=k^{2} \psi(k), \quad \psi(k) \in D\left(A_{0}^{*}\right), \quad k^{2} \in \mathbb{C}-\mathbb{R}, \quad \operatorname{Im}(k)>0,
$$

tem uma única solução dada por $\psi(k, x)=e^{i k|x|}, \operatorname{Im}(k)>0$. Logo para $k^{2}= \pm i$, com $\operatorname{Im}(\sqrt{ \pm i})>0$ temos os elementos de deficiência

$$
\psi_{ \pm}(x)=\frac{i}{2 \sqrt{ \pm i}} e^{i \sqrt{ \pm i}|x|}, \quad \operatorname{Im}(\sqrt{ \pm i})>0
$$

Notamos de $(5.17)$ e $(5.18)$ que $\widehat{\psi_{ \pm i}}(\xi)=\frac{1}{\xi^{2} \pm i}$. Assim, pela Teoria de Von Neumann para a extensão de operadores simétricos [45], todas as extensões autoadjuntas $A_{\theta, 0}$ de $A_{0}$ são dadas pela família a um parâmetro

$$
\begin{aligned}
& D\left(A_{\theta, 0}\right)=\left\{g+c \psi_{+}+c e^{i \theta} \psi_{-} \mid g \in D\left(A_{0}\right), c \in \mathbb{C}\right\} \\
& A_{\theta, 0}\left(g+c \psi_{+}+c e^{i \theta} \psi_{-}\right)=A_{0} g+i c \psi_{+}-i c e^{i \theta} \psi_{-}
\end{aligned}
$$

com $\psi_{+}, \psi_{-}$definidos em (5.18) e $\theta \in[0,2 \pi)$. Além disso, se definimos $\phi(0 \pm)=$ $\lim _{\epsilon \downarrow 0} \phi( \pm \epsilon)$ obtemos que para $\xi=g+c \psi_{+}+c e^{i \theta} \psi_{-}$

$$
\begin{aligned}
& \xi^{\prime}(0+)-\xi^{\prime}(0-)=-c\left(1+e^{i \theta}\right) \\
& =\gamma\left[g(0)+c \psi_{+}(0)+c e^{i \theta} \psi_{-}(0)\right]=\gamma \xi(0)
\end{aligned}
$$

onde $\gamma$ vem dado por

$$
\gamma(\theta)=\frac{-\sqrt{2}[1+\cos (\theta)+\operatorname{sen}(\theta)]}{1+\operatorname{sen}(\theta)} .
$$

Notemos que para $\theta=\frac{3 \pi}{2}$, a função $\gamma$ tem uma assintota vertical, como se mostra no seguinte gráfico 


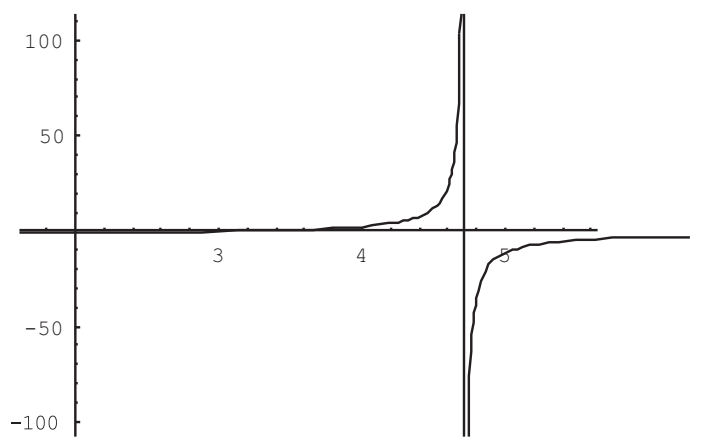

O teorema seguinte mostra que todas as extensões autoadjuntas $A_{\theta, 0}$ de $A_{0}$ podem ser parametrizadas em termos do paramemetro $\gamma$, mais precisamente:

Teorema 5.2.1. Todas as extensões autoadjuntas de $A_{0}$, para $-\infty<\gamma \leq \infty$ são dadas por

$$
\begin{aligned}
& -\Delta_{\gamma} \equiv A_{\gamma, 0}=-\frac{d^{2}}{d x^{2}}, \\
& D\left(-\Delta_{\gamma}\right)=\left\{g \in H^{1}(\mathbb{R}) \cap H^{2}(\mathbb{R}-\{0\}) \mid g^{\prime}(0+)-g^{\prime}(0-)=\gamma g(0)\right\},
\end{aligned}
$$

o caso $\gamma=0$ resulta no operador de Laplace no espaço $L^{2}(\mathbb{R})$, isto é

$$
-\Delta_{0}=-\frac{d^{2}}{d x^{2}}, \quad D\left(-\Delta_{0}\right)=H^{2}(\mathbb{R}) .
$$

O caso $\gamma=\infty$, divide a reta real em dois intervalos $(-\infty, 0),(0, \infty)$, isto acontece devido à aparição da condição de fronteira do tipo Dirichlet no ponto 0 , isto é

$$
\begin{aligned}
D\left(-\Delta_{\infty}\right) & =\left\{g \in H^{1}(\mathbb{R}) \cap H^{2}(\mathbb{R}-\{0\}) \mid g(0)=0\right\}, \\
& =H_{0}^{2}((-\infty, 0)) \oplus H_{0}^{2}((0, \infty)), \\
& -\Delta_{\infty}=\left(-\Delta_{D-}\right) \oplus\left(-\Delta_{D+}\right),
\end{aligned}
$$

onde $\left(-\Delta_{D \pm}\right)$ denota o Laplaciano de Dirichlet sobre $(-\infty, 0),(0, \infty)$ respectivamente, (ver [46], pag 253), com $D\left(-\Delta_{D_{+}}\right)=H_{0}^{2}((0, \infty)), \quad D\left(-\Delta_{D_{-}}\right)=H_{0}^{2}((-\infty, 0))$.

Demonstração. Da equação (5.21) segue que $A_{\theta, 0} \subset-\Delta_{\gamma}$, com $\gamma=\gamma(\theta)$ dado pela equação (5.22). Mas $-\Delta_{\gamma}$ é simétrico sobre seu correspondente domínio $D\left(-\Delta_{\gamma}\right)$ para todo $-\infty<\gamma \leq+\infty$, o qual implica a relação

$$
A_{\theta, 0} \subset-\Delta_{\gamma} \subset\left(-\Delta_{\gamma}\right)^{*} \subset A_{\theta, 0}
$$

o que completa a prova. 
O seguinte teorema nos da uma fórmula explícita para a resolvente do operador $A_{\gamma, 0}$ e é baseado na fórmula de Krein (ver [3]).

Teorema 5.2.2. A resolvente de $\Delta_{\gamma}$ é dada por

$$
\begin{aligned}
& \left(\Delta_{\gamma}-k^{2}\right)^{-1}=\left(-\Delta-k^{2}\right)^{-1}-\frac{2 \gamma k}{i \gamma+2 k}\left(\overline{G_{k}(\cdot)}, \cdot\right) G_{k}(\cdot), \\
& k^{2} \in \rho\left(\Delta_{\gamma}\right), \quad \operatorname{Im}(k)>0, \quad-\infty<\gamma \leq \infty
\end{aligned}
$$

onde

$$
G_{k}(x)=\frac{i}{2 k} e^{i k|x|}, \quad \operatorname{Im}(k)>0,
$$

$\operatorname{satisfaz}\left(-\Delta-k^{2}\right)^{-1} f=G_{k} * f$.

Sobre as propriedades espectrais do operador $\Delta_{\gamma}$ temos o seguinte teorema.

Teorema 5.2.3. Seja $-\infty<\gamma \leq \infty$. Então o espectro essencial de $\Delta_{\gamma}$ é absolutamente contínuo e

$$
\sigma_{e s s}\left(\Delta_{\gamma}\right)=[0, \infty)
$$

Se $-\infty<\gamma<0, \Delta_{\gamma}$, tem exatamente um autovalor negativo e simples, isto é seu espectro pontual $\sigma_{p}\left(A_{\gamma, 0}\right)$ vem dado por

$$
\sigma_{p}\left(\Delta_{\gamma}\right)=\left\{-\frac{\gamma^{2}}{4}\right\}
$$

onde

$$
\psi_{\gamma}(x)=\sqrt{\frac{-\gamma}{2}} e^{\frac{\gamma|x|}{2}}
$$

é a autofunção estritamente positiva e normalizada associada. Se $\gamma \geq 0, \Delta_{\gamma}$ não tem autovalores de tipo finito, isto é

$$
\sigma_{p}\left(\Delta_{\gamma}\right)=\emptyset
$$

Observação 5.2.1. O Teorema 5.2.2 pode ser usado para mostrar que as famílias $Z \rightarrow \mathcal{L}_{i, Z}$ definidas em (5.49) são analíticas. Teorema 5.2.3 é essencial para mostrar que o problema de Cauchy associado a equação (5.2) é bem posto em $H^{1}(\mathbb{R})$. 


\subsection{Boa colocação local e global para a equação CQD-S}

Sobre a boa colocação local (no sentido da definição 4.1.1) do problema de valor inicial

$$
\begin{aligned}
& i u_{t}+u_{x x}+Z \delta(x) u+u\left(|u|^{2}+|u|^{4}\right)=0, \\
& u(0) \in H^{1}(\mathbb{R})
\end{aligned}
$$

temos o seguinte Teorema:

Teorema 5.3.1. O problema de Cauchy (5.32), é localmente bem posto em $H^{1}(\mathbb{R})$. Além disso, se o dado inicial u(0) é par então a solução u(t) também é par.

Demonstração. A prova segue do Teorema 3.7.1 em [20] aplicado a nosso problema. De fato do Teorema 5.2.3 temos que o operador $-\Delta_{-Z} \geq-\beta$, $\operatorname{com} \beta=\frac{Z^{2}}{4}$ para $Z>0$ e $\beta=0$ se $Z<0$. Assim, para o operador auto-adjunto $\mathcal{A} \equiv \Delta_{-Z}-\beta$ sobre o espaço $X=L^{2}(\mathbb{R})$ com domínio $D(\mathcal{A})=D\left(-\Delta_{-Z}\right)$ temos que $\mathcal{A} \leq 0$. Além disso, em nosso caso é possível considerar o espaço $X_{\mathcal{A}}=H^{1}(\mathbb{R})$ com norma

$$
\|u\|_{X_{\mathcal{A}}}=\left\|u_{x}\right\|_{L^{2}}^{2}+(\beta+1)\|u\|_{L^{2}}^{2}-Z|u(0)|^{2},
$$

a qual é equivalente à norma usual de $H^{1}(\mathbb{R})$. Portanto, não é difícil ver que a unicidade de soluções e as condições (3.7.1), (3.7.3)-(3.7.6) em [20] são satisfeitas com $r=\rho^{\prime}=2$. Finalmente, a condição (3.7.2) em [20] é válida pois $\mathcal{A}$ é um operador auto-adjunto no espaço $L^{2}(\mathbb{R})$.

Já, para um resultado sobre existência de soluções globais (definidas para todo tempo) observamos primeiramente que os funcionais $E$ e $Q$, definidos por:

$$
\begin{aligned}
& E(u)=\frac{1}{2} \int\left|u_{x}\right|^{2} d x-\frac{Z}{2} \int \delta(x)|u(x)|^{2} d x-\frac{1}{4} \int|u|^{4} d x-\frac{1}{6} \int|u|^{6} d x, \\
& Q(u)=\frac{1}{2} \int|u|^{2} d x .
\end{aligned}
$$

são quantidades conservadas pelo fluxo da equação CQD-S. Com isto, temos o seguinte teorema. 
Teorema 5.3.2. Seja $\phi \in H^{1}(\mathbb{R})$. Então a solução u do problema (5.32) é globalmente definida sempre que a norma do dado inicial $u(0)=\phi$, seja pequena no espaço $L^{2}(\mathbb{R})$.

Demonstração. Como sabemos (ver seção 4.1), basta mostrar que a norma $H^{1}(\mathbb{R})$ da solução $u(t)$, não explode em tempo finito. Assim, usando a notação do teorema anterior e a desigualdade de Gagliardo-Nirenberg (no caso da reta) da mesma forma que foi feito no Teorema 4.1.5, temos que

$$
\begin{aligned}
\left\|u_{x}(t)\right\|_{L^{2}}^{2}-Z|u(0)|^{2} & =2 E(u)+\frac{1}{2}\|u\|_{L^{4}}^{4}+\frac{1}{3}\|u\|_{L^{6}}^{6} \\
& \leq 2 E(u)+\frac{d_{0}}{4}\left(\left\|u_{x}\right\|_{L^{2}}^{2}+\|u\|_{L^{2}}^{6}\right)+\frac{c_{0}}{3}\left\|u_{x}\right\|_{L^{2}}^{2}\|u\|_{L^{2}}^{4} \\
& \leq 2 E(u)+\frac{d_{0}}{4}\|u\|_{L^{2}}^{6}+\left(\frac{d_{0}}{4}+\frac{c_{0}}{3}\|u\|_{L^{2}}^{4}\right)\left\|u_{x}\right\|_{L^{2}}^{2},
\end{aligned}
$$

de onde deduzimos da conservação das quantidades $E$ e $Q$ que

$$
\left(1-h\left(\|\phi\|_{L^{2}}\right)\right)\left\|u_{x}\right\|_{L^{2}}-Z|u(0)|^{2} \leq 2 E(\phi)+2 d_{0} Q(\phi)^{3},
$$

$\operatorname{com} h(x)=\frac{d_{0}}{4}+\frac{c_{0}}{3} x^{4}$. Agora, se $\phi$ satisfaz a condição $1-h\left(\|\phi\|_{L^{2}}\right)>0$, obtemos então que

$$
\left\|u_{x}(t)\right\|_{L^{2}}^{2}-\frac{Z|u(0)|^{2}}{1-h\left(\|\phi\|_{L^{2}}\right)} \leq \frac{2 E(\phi)+2 d_{0} Q(\phi)^{3}}{1-h\left(\|\phi\|_{L^{2}}\right)} .
$$

Da anterior desigualdade segue a limitação uniforme da quantidade

$$
\left\|u_{x}(t)\right\|_{L^{2}}^{2}-Z|u(0)|^{2}
$$

isto finaliza a prova do teorema.

\subsection{Existência de picos solitários para a equação CQD-S}

Nesta seção vamos encontrar soluções explícitas do tipo (5.1) para a equação (5.2). Assim, substituindo (5.1) em (5.2) temos que o perfil $\phi$ deve satisfazer no sentido distribucional a equação elíptica semilinear,

$$
\phi^{\prime \prime}+w \phi+Z \delta(x) \phi+\phi^{3}+\phi^{5}=0 .
$$


Como queremos que esta solução esteja no domínio do operador $-\frac{d^{2}}{d x^{2}}-Z \delta$, isto é, no conjunto

$$
D:=\left\{u \in H^{1}(\mathbb{R}) \cap H^{2}(\mathbb{R}-\{0\}) \mid u^{\prime}(0+)-u^{\prime}(0-)=-Z u(0)\right\},
$$

então precisamos que a solução da equação (5.37) satisfaça no ponto $x=0$ a condição de salto sobre a primeira derivada, $\phi^{\prime}(0+)-\phi^{\prime}(0-)=-Z \phi(0)$. De fato seguindo as idéias dadas em [25] é possível mostrar o seguinte lema.

Lema 5.4.1. Seja $Z \in \mathbb{R} e-w>\frac{Z^{2}}{4}$. Então qualquer solução $g \in H^{1}(\mathbb{R})$ de (5.37), satisfaz as seguintes propriedades

$$
\begin{aligned}
& g \in C^{j}(\mathbb{R}-\{0\}) \cap C(\mathbb{R}), \quad j=1,2 . \\
& -g^{\prime \prime}-w g-g^{3}-g^{5}=0, \quad \text { para } x \neq 0 . \\
& g(0+)-g(0-)=-Z g(0) . \\
& g^{\prime}(x), g(x) \rightarrow 0, \quad \text { se }|x| \rightarrow \infty .
\end{aligned}
$$

Demonstração. ver Lema 3.1 em [25].

Agora, vamos mostrar como é possível construir uma solução do problema (5.37) $(\operatorname{com} Z \neq 0)$ da solução do problema com $Z=0$. Para este fim seja então $\alpha=\frac{-1}{4 w} \mathrm{e}$ $\beta=\frac{\sqrt{9-48 w}}{-12 w}$, logo da simetria por traslações da equação (5.5) e da fórmula (5.6) vemos que a função dada por

$$
\phi_{w, b}(x)=[\alpha+\beta \cosh (2 \sqrt{-w}(x+b))]^{-\frac{1}{2}}
$$

satisfaz a equação (5.5) para todo $b \in \mathbb{R}$. Do Lema 5.4.1 é claro que a solução $\phi$ da equação (5.37) (com $Z \neq 0$ ), deve ser dada do modo seguinte

$$
\phi(x)= \begin{cases}\phi_{w, b}(x) & \text { se } x>0 \\ \phi_{w, r}(x) & \text { se } x<0 .\end{cases}
$$

Para algum $b$ e $r \in \mathbb{R}$. Como a função $\phi$ deve ser contínua então necessariamente $r=|b|$. Agora se $r=b$ a função $\phi$ em (5.41) é uma solução suave do problema (5.37) 
com $Z=0$. No caso $r=-b \neq 0, \phi$ é uma solução do problema (5.37) para $Z \neq 0$, neste caso a função $\phi$ em (5.41) pode ser reescrita na forma $\phi(x)=\phi_{w, b}(|x|)$. Pela paridade da função $\phi$, vemos que a condição de salto em $(5.39 \mathrm{c})$ se reduz à condição $\phi^{\prime}(0+)=-\frac{Z}{2} \phi(0)$. Assim, se $x \geq 0$ temos que $\phi(x)=\phi_{w, b}(x)$ logo de (5.40) deduzimos para $x>0$

$$
\phi_{w, b}^{\prime}(x)=-\frac{\beta \sqrt{-w}}{2}[\alpha+\beta \cosh (2 \sqrt{-w}(x+b))]^{-\frac{3}{2}} \operatorname{senh}(2 \sqrt{-w}(x+b)) .
$$

Usando (5.40) e (5.42) deduzimos que $Z, b, w$ satisfazem a seguinte relação

$$
\frac{\beta \operatorname{senh}(2 \sqrt{-w} b)}{\alpha+\beta \cosh (2 \sqrt{-w} b)}=\frac{Z}{2 \sqrt{-w}}
$$

É facíl ver que a função $b \rightarrow R(b)=\frac{\beta \sinh (2 \sqrt{-w} b)}{\alpha+\beta \cosh (2 \sqrt{-w} b)}$, é um difeomorfismo crescente do intervalo $(-\infty, \infty)$, no intervalo $(-1,1)$. Para $w=-1$ temos o seguinte gráfico:

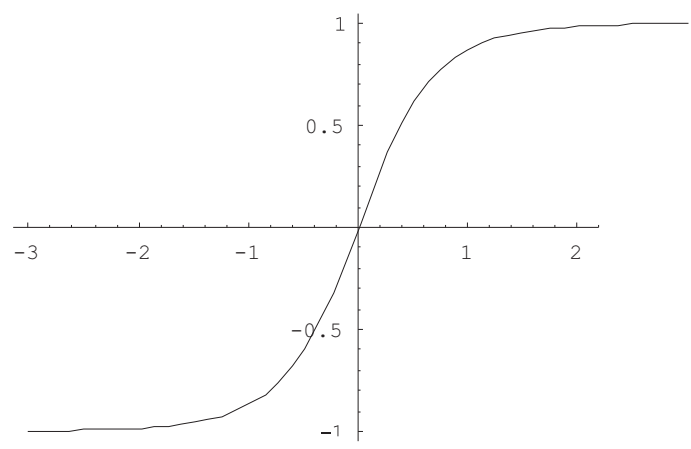

Portanto da equação (5.43) deduzimos que $w, Z$ devem satisfazer a relação

$$
\frac{Z^{2}}{4}<-w
$$

Como $R$ é um difeomorfismo então existe uma função suave $R^{-1}:(-1,1) \rightarrow(-\infty, \infty)$, tal que $b=R^{-1}\left(\frac{Z}{2 \sqrt{-w}}\right)$. Logo obtemos que a função dada por

$$
\phi_{w, Z}(x)=\left[-\frac{1}{4 w}-\frac{\sqrt{9-48 w}}{12 w} \cosh \left(2 \sqrt{-w}\left(|x|+R^{-1}\left(\frac{Z}{2 \sqrt{-w}}\right)\right)\right)\right]^{-\frac{1}{2}}
$$

é a solução positiva associada à equação (5.37). Por exemplo se $w=-3, Z=-2$, então obtemos o seguinte gráfico para as soluções dadas em (5.45) 


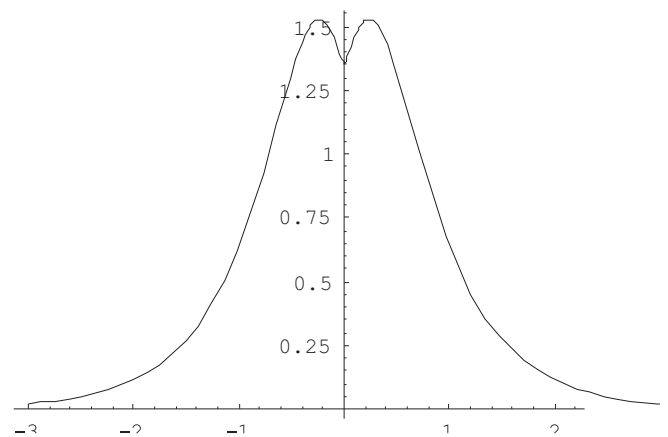

Já se $w=-3, Z=2$, temos

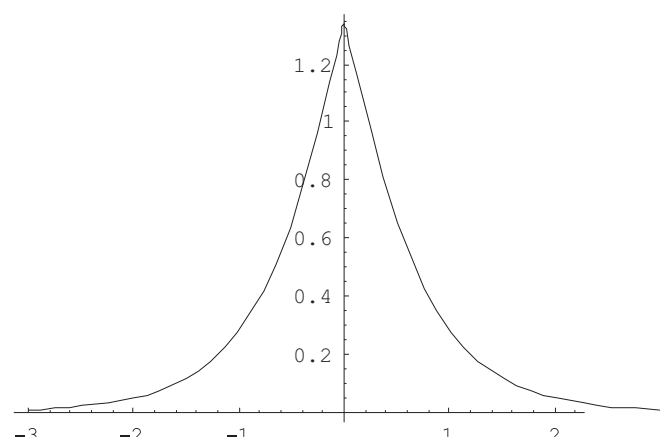

Observação 5.4.1. Em geral para $Z<0, w<0$ satisfazendo a relação (5.44), $\phi_{w, Z}$ tem o perfil dado pela primeira figura acima. Para $Z>0, w<0$ satisfazendo a relação (5.44), $\phi_{w, Z}$ tem o perfil dado pela segunda figura acima.

Usando o Lema 5.4.1 e seguindo as idéias expostas em [25], é possível mostrar que $\phi_{w, Z}$ dada em (5.45) é a única solução do problema (5.37). Mais precisamente

Lema 5.4.2. Seja $Z \in \mathbb{R}-\{0\} e-w>\frac{Z^{2}}{4}$. Então (5.37) tem uma única solução não negativa não trivial. Deduzimos que o conjunto de todas as soluções de tipo standing waves para (5.32) são dadas por

$$
\left\{e^{i \theta} \phi_{w, Z} \mid \theta \in \mathbb{R}\right\} .
$$




\subsection{Estabilidade de standig waves para a equação CQD-S}

Nesta seção estudaremos a estabilidade da órbita $\Omega_{\phi_{w, Z}}=\left\{e^{i \theta} \phi_{w, Z} \mid \theta \in[0,2 \pi)\right\}$,gerada pela curva suave $w \rightarrow \phi_{w, Z}$, com $\phi_{w, Z}$ dada em (5.45). Notamos que a equação (5.32) não é invariante por translações. Assim, a nossa definição de estabilidade é dada por:

Definição 5.5.1. Para $\eta>0$, seja $\phi$ a solução de (5.37) e definamos

$$
U_{\eta}(\phi)=\left\{v \in H^{1}(\mathbb{R}) \mid \inf _{\theta \in \mathbb{R}}\left\|v-e^{-i \theta} \phi\right\|_{1}<\eta\right\} .
$$

Dizemos que a standing wave $e^{-i w t} \phi$ é orbitalmente estável em $H^{1}(\mathbb{R})$, se para todo $\epsilon>0$ existe $\eta>0$ tal que para todo $u_{0} \in U_{\eta}(\phi)$, a solução $u(t)$ de (5.2) com $u(0)=u_{0}$ satisfaz $u(t) \in U_{\epsilon}(\phi)$ para todo $t \in \mathbb{R}$. Caso contrário, $e^{-i w t} \phi$ é dita orbitalmente instável.

Agora, para $u \in H^{1}(\mathbb{R})$ escrevemos $u=u_{1}+i u_{2} \operatorname{com} u_{1}=\operatorname{Re}(u)$ e $u_{2}=\operatorname{Im}(u)$. Seja $H_{w, Z}$ definido por

$$
H_{w, Z}(u)=\mathcal{L}_{1, Z} u_{1}+i \mathcal{L}_{2, Z} u_{2}
$$

onde os operadores lineares $\mathcal{L}_{i, Z}, i=1,2$ são definidos sobre o domínio:

$$
\mathcal{D} \equiv D\left(\mathcal{L}_{i, Z}\right)=\left\{g \in H^{1}(\mathbb{R}) \cap H^{2}(\mathbb{R}-\{0\}) \mid g^{\prime}(0+)-g^{\prime}(0-)=-Z g(0)\right\}
$$

da seguinte maneira, para $g \in \mathcal{D}$

$$
\begin{aligned}
& \mathcal{L}_{1, Z} g=-\frac{d^{2}}{d x^{2}} g-w g-3 \phi_{w, Z}^{2} g-5 \phi_{w, Z}^{4} g \\
& \mathcal{L}_{2, Z} g=-\frac{d^{2}}{d x^{2}} g-w g-\phi_{w, Z}^{2} g-\phi_{w, Z}^{4} g .
\end{aligned}
$$

aqui $-w>0$ e $\phi_{w, Z}$ definida em (5.45). Não é difícil ver que os operadores $\mathcal{L}_{1, Z}$ e $\mathcal{L}_{2, Z}$ são auto-adjuntos sobre $\mathcal{D}$, ainda mais eles aparecem naturalmente como a segunda variação associada ao funcional

$$
G_{w, Z}=E-w Q,
$$


com $E$ e $Q$ dados em (5.33). Mais exatamente, seja $u=\zeta+i \psi \operatorname{com} \zeta, \psi \in \mathcal{D}$ e $v=v_{1}+i v_{2} \in H^{1}(\mathbb{R})$, então

$$
\left\langle G_{w, Z}^{\prime \prime}\left(\phi_{w, Z}\right) u, v\right\rangle=\left\langle H_{w, Z} u, v\right\rangle=\left\langle\mathcal{L}_{1, Z} \zeta+i \mathcal{L}_{2, Z} \psi, v\right\rangle=\left\langle\mathcal{L}_{1, Z} \zeta, v_{1}\right\rangle+\left\langle\mathcal{L}_{2, Z} \psi, v_{2}\right\rangle
$$

É importante observar que os funcionais $E, Q$ definidos em (5.33) são invariantes pelo grupo de rotações $T(\theta) u=e^{i \theta} u$ e formalmente conservados pelo fluxo da CQD-S. Agora vamos analizar as propriedades espectrais dos operadores $\mathcal{L}_{1, Z}$ e $\mathcal{L}_{2, Z}$. Sobre o operador $\mathcal{L}_{2, Z}$ temos o seguinte resultado o qual segue do Teorema 5.2.3 e da positividade da solução $\phi_{w, Z}$.

Lema 5.5.1. Sejam $w<0, Z \in \mathbb{R}-\{0\} e-w>\frac{Z^{2}}{4}$. Então, $\mathcal{L}_{2, Z}$ é um operador não negativo com espectro dado por

$$
\sigma_{p}\left(\mathcal{L}_{2, Z}\right)=\{0\}, \quad \sigma_{\text {ess }}\left(\mathcal{L}_{2, Z}\right)=[-w, \infty),
$$

onde zero é um autovalor simples com autofunção $\phi_{w, Z}$.

Agora estudaremos as propriedades espectrais do operador $\mathcal{L}_{1, Z}$, as quais são mais delicadas. Começamos com o seguinte lema.

Lema 5.5.2. Sejam $w<0, Z \in \mathbb{R}-\{0\} e-w>\frac{Z^{2}}{4}$. Se $\lambda$ é um autovalor simples de $\mathcal{L}_{1, Z}$ então a autofunção associada é par ou ímpar.

Demonstração. Sejam $w<0 v \in D\left(\mathcal{L}_{1, Z}\right)-\{0\}$ tal que $\mathcal{L}_{1, Z} v=\lambda v$. Do fato que $\varphi_{w, Z}$ é par, obtemos que $g(x):=v(-x)$ tambem satisfaz $\mathcal{L}_{1, Z} g=\lambda g$. Como $\lambda$ é um autovalor simples para o operador $\mathcal{L}_{1, Z}$, então existe $\beta \in \mathbb{R}$ tal que $v(x)=\beta v(-x)$ para $x \in \mathbb{R}$. Se $v(0) \neq 0$ então $\beta=1$ assim $v$ é par. Se $v(0)=0$ então do Lema 5.4.1 ou da definição de $\mathcal{D}$ obtemos que $v^{\prime}(x)$ existe para todo $x \in \mathbb{R}$, portanto temos que $v^{\prime}(0)=-\beta v^{\prime}(0)$ logo do principio de unicidade de Cauchy $v^{\prime}(0) \neq 0$ (em outro caso $v \equiv 0)$. Assim $\beta=-1$ de onde $v$ é ímpar.

Lema 5.5.3. Sejam $w<0, Z \in \mathbb{R}-\{0\} e-w>\frac{Z^{2}}{4}$. Então $\mathcal{L}_{1, Z}$ tem núcleo trivial. 
Demonstração. Notamos primeiro que todo elemento $u$ no núcleo do operador $\mathcal{L}_{1, Z}$ é uma solução do problema

$$
\left\{\begin{array}{l}
\mathcal{L}_{1, Z} \phi(x)=0, \quad x>0 . \\
\phi \in L^{2}((0, \infty)) .
\end{array}\right.
$$

É conhecido (ver Teorema 8.1 em [21]) que o problema linear (5.52) tem dimensão um. Como $\phi_{w, Z}^{\prime}$ satisfaz o problema (5.52), então é claro que se $v \in \mathcal{D}\left(\mathcal{L}_{1, Z}\right)$ e $\mathcal{L}_{1, Z} v=0$ então existe $\alpha \in \mathbb{R}$ tal que $v(x)=\alpha \phi_{w, Z}^{\prime}(x)$, para $x>0$. Um argumento análogo aplicado ao problema

$$
\left\{\begin{array}{l}
\mathcal{L}_{1, Z} \phi(x)=0, \quad x<0 . \\
\phi \in L^{2}((-\infty, 0))
\end{array}\right.
$$

nos permite concluir a existência de um número real $\beta$ tal que $v(x)=\beta \phi_{w, Z}^{\prime}(x)$, para $x<0$. Pela continuidade de $v$ e a paridade da função $\phi_{w, Z}$ deduzimos que $\alpha=-\beta$. Por outro lado, como $v \in \mathcal{D}\left(\mathcal{L}_{1, Z}\right)$, então $v^{\prime}(0+)-v^{\prime}(0-)=-Z v(0)$, de onde

$$
\begin{aligned}
v^{\prime}(0+)-v^{\prime}(0-) & =\alpha \phi_{w, Z}^{\prime \prime}(0+)+\alpha \phi_{w, Z}^{\prime \prime}(0-), \\
& =-Z \alpha \phi_{w, Z}^{\prime}(0+) .
\end{aligned}
$$

Assim, se $\alpha \neq 0$ então concluimos que $\phi_{w, Z}^{\prime \prime}(0+)=-\frac{Z}{2} \phi_{w, Z}^{\prime}(0+)$ (pois de $(5.39 \mathrm{~b})$ $\left.\phi^{\prime \prime}(0+)=\phi^{\prime \prime}(0-)\right)$. Agora, multiplicando a equação (5.39b) por $g^{\prime}$ e integrando no intervalo $(0, R)$, obtemos

$$
-\frac{1}{2}\left(g^{\prime}(R)\right)^{2}+\frac{1}{2}\left(g^{\prime}(0+)\right)^{2}-F(g(R))+F(g(0+))=0,
$$

com $F(s)=w \frac{s^{2}}{2}+\frac{s^{4}}{4}+\frac{s^{6}}{6}$, fazendo $R \rightarrow \infty$ e usando (5.39d) deduzimos então que,

$$
\frac{1}{2}\left(g^{\prime}(0+)\right)^{2}+F(g(0+))=0
$$

Agora, como $\phi_{w, Z}$ satisfaz a equação (5.39b) então de (5.56) obtemos que $\frac{1}{2}\left(\phi_{w, Z}^{\prime}(0+)\right)^{2}=$ $-F\left(\phi_{w, Z}(0)\right)$, e como $\phi_{w, Z}$ é par temos também que $\phi_{w, Z}^{\prime}(0+)=\frac{-Z}{2} \phi_{w, Z}(0)$. Logo deduzimos que $\phi_{w, Z}(0) \equiv c_{0}>0$ é um zero do seguinte polinômio

$$
P(c)=\frac{Z^{2}+4 w}{8} c^{2}+\frac{c^{4}}{4}+\frac{c^{6}}{6}
$$


de onde

$$
c_{0}=\sqrt{\frac{-6+\sqrt{36-48\left(Z^{2}+4 w\right)}}{8}} .
$$

Assim usando a equação (5.37) observamos que $\phi_{w, Z}$ satisfaz

$$
\begin{aligned}
\frac{Z^{2}}{4} c_{0} & =\frac{Z^{2}}{4} \phi_{w, Z}(0)=-\frac{Z}{2} \phi_{w, Z}^{\prime}(0+)=\phi_{w, Z}^{\prime \prime}(0+) \\
& =\lim _{x \rightarrow 0+} \phi_{w, Z}^{\prime \prime}(x)=-w c_{0}-c_{0}^{3}-c_{0}^{5} .
\end{aligned}
$$

Logo considerando os zeros não nulos de

$$
\frac{Z^{2}}{4} s=-w s-s^{3}-s^{5}
$$

isto é

$$
s_{0}= \pm \sqrt{\frac{-4+\sqrt{16-16\left(Z^{2}+4 w\right)}}{8}}
$$

vemos que $\left|s_{0}\right|<\left|c_{0}\right|$, isto é uma contradição. Portanto $\alpha=0$ de onde $v \equiv 0$ e assim $\mathcal{L}_{1, Z}$ tem núcleo trivial.

\subsubsection{Autovalores do operador $\mathcal{L}_{1, Z}$}

Nesta subseção usamos a teoria de perturbação analítica para estudar o número de autovalores negativos de $\mathcal{L}_{1, Z}$ para $Z \neq 0$. Como o domínio dos operadores $\mathcal{L}_{1, Z}$ varia com $Z$ usaremos a teoria de perturbação analítica para operadores lineares (ver Kato [35] e Reed-Simon [46]), vamos dividir nosso estudo em vários items,

(I) Pelas equações $(5.43),(5.45)$ sabemos que para $-w>\frac{Z^{2}}{4}$, obtemos que

$$
\lim _{Z \rightarrow 0} \phi_{w, Z}(x)=\phi_{w, 0}(x), \quad \text { para } x \in \mathbb{R}
$$

onde $\phi_{w, 0}$ denota o perfil da standing wave associada com a CQ-S dada pela equação (5.6).

(II) Os operadores lineares $\mathcal{L}_{i, Z}$, em (5.49), $i=1,2$, são operadores auto-adjuntos sobre $L^{2}(\mathbb{R})$, associados com as seguintes formas bilineares, para $p, v \in H^{1}(\mathbb{R})$,

$$
\begin{aligned}
& \mathcal{Q}_{w, Z}^{1}(v, p)=\int_{\mathbb{R}} v_{x} p_{x} d x-\int_{\mathbb{R}}\left(w+3 \phi_{w, Z}^{2}+5 \phi_{w, Z}^{4}\right) v p d x-Z v(0) p(0) \\
& \mathcal{Q}_{w, Z}^{2}(v, p)=\int_{\mathbb{R}} v_{x} p_{x} d x-\int_{\mathbb{R}}\left(w+\phi_{w, Z}^{2}+\phi_{w, Z}^{4}\right) v p d x-Z v(0) p(0) .
\end{aligned}
$$


Como estas formas tem o mesmo domínio $D\left(\mathcal{Q}_{w, Z}^{i}\right)=H^{1}(\mathbb{R})$ e elas são simétricas, limitadas por abaixo e fechadas, temos então pela teoria de representação de formas para operadores (ver primeiro teorema de representação em Kato [35], VI. Seção 2.1), existem operadores autoadjuntos $\widetilde{\mathcal{L}}_{1}: D\left(\widetilde{\mathcal{L}}_{1}\right) \subset L^{2}(\mathbb{R}) \rightarrow L^{2}(\mathbb{R})$ e $\widetilde{\mathcal{L}}_{2}: D\left(\widetilde{\mathcal{L}}_{2}\right) \subset L^{2}(\mathbb{R}) \rightarrow L^{2}(\mathbb{R})$ tal que

$$
\begin{aligned}
& D\left(\widetilde{\mathcal{L}}_{1}\right)=\left\{v \in H^{1}(\mathbb{R}) \mid \exists p \in L^{2}(\mathbb{R}) \text { s.t. } \forall z \in H^{1}(\mathbb{R}), \mathcal{Q}_{w, Z}^{1}(v, z)=(p, z)\right\} \\
& D\left(\widetilde{\mathcal{L}}_{2}\right)=\left\{v \in H^{1}(\mathbb{R}) \mid \exists p \in L^{2}(\mathbb{R}) \text { s.t. } \forall z \in H^{1}(\mathbb{R}), \mathcal{Q}_{w, Z}^{2}(v, z)=(p, z)\right\}
\end{aligned}
$$

e para $v \in D\left(\widetilde{\mathcal{L}}_{1}\right)$ (respectivamente $v \in D\left(\widetilde{\mathcal{L}}_{2}\right)$ ) definimos $\widetilde{\mathcal{L}}_{1} v \equiv p$ (respectivamente $\left.\widetilde{\mathcal{L}}_{2} v \equiv p\right)$, onde $p$ é a única função de $L^{2}(\mathbb{R})$ que satisfaz $\mathcal{Q}_{w, Z}^{1}(v, z)=(p, z)$ (respectivamente $\mathcal{Q}_{w, Z}^{2}(v, z)=(p, z)$ ) para todo $z \in H^{1}(\mathbb{R})$. Agora mostraremos explicitamente os operadores $\widetilde{\mathcal{L}}_{1}$ e $\widetilde{\mathcal{L}}_{2}$.

Lema 5.5.4. O dominio de $\widetilde{\mathcal{L}}_{1}$ e $\widetilde{\mathcal{L}}_{2}$ em $L^{2}(\mathbb{R})$ é

$$
D_{Z}:=\left\{\zeta \in H^{1}(\mathbb{R}) \cap H^{2}(\mathbb{R}-\{0\}) \mid \zeta^{\prime}(0+)-\zeta^{\prime}(0-)=-Z \zeta(0)\right\},
$$

e para $v$ em $D_{Z}$ os operadores são dados por

$$
\begin{aligned}
& \widetilde{\mathcal{L}}_{1} v=-\frac{d^{2}}{d x^{2}} v-w v-3 \phi_{w, Z}^{2} v-5 \phi_{w, Z}^{4} v, \\
& \widetilde{\mathcal{L}}_{2} v=-\frac{d^{2}}{d x^{2}} v-w v-\phi_{w, Z}^{2} v-\phi_{w, Z}^{4} v .
\end{aligned}
$$

Demonstração. Vamos fazer a prova para o operador $\widetilde{\mathcal{L}}_{1}$ (a prova no caso do operador $\widetilde{\mathcal{L}}_{2}$ é similar). Vamos decompor $\mathcal{Q}_{w, Z}^{1}$ na forma $\mathcal{Q}_{w, Z}^{1}=\mathcal{Q}_{Z}^{1}+\mathcal{Q}_{w}^{1}$ com $\mathcal{Q}_{Z}^{1}: H^{1}(\mathbb{R}) \times H^{1}(\mathbb{R}) \rightarrow \mathbb{R}$ e $\mathcal{Q}_{w}^{1}: L^{2}(\mathbb{R}) \times L^{2}(\mathbb{R}) \rightarrow \mathbb{R}$ dadas por

$$
\begin{aligned}
& \mathcal{Q}_{Z}^{1}(v, z)=\int_{\mathbb{R}} v_{x} z_{x} d x-Z v(0) z(0), \\
& \mathcal{Q}_{w}^{1}(v, z)=-\int_{\mathbb{R}}\left(w+3 \phi_{w, Z}^{2}+5 \phi_{w, Z}^{4}\right) v z d x .
\end{aligned}
$$

Denotemos por $\Upsilon_{1}$ (respectivamete $\Upsilon_{2}$ ) o operador auto-adjunto sobre $L^{2}(\mathbb{R})$ associado com $\mathcal{Q}_{Z}^{1}\left(\right.$ respectivamente $\left.\mathcal{Q}_{w}^{1}\right)$. Claramente $D\left(\Upsilon_{2}\right)=L^{2}(\mathbb{R})$ e $D\left(\Upsilon_{1}\right)=$ $D\left(\widetilde{\mathcal{L}}_{1}\right)$. Vamos mostrar agora que $\Upsilon_{1}$ é uma extensão auto-adjunta do operador 
$A_{0}$ definido em (5.14), de fato, seja $v \in H^{2}(\mathbb{R})$ tal que $v(0)=0$, e definamos $w \equiv-v_{x x} \in L^{2}(\mathbb{R})$, então para todo $z \in H^{1}(\mathbb{R})$ temos que $\mathcal{Q}_{Z}^{1}(v, z)=(w, z)$. Então $v \in D\left(\Upsilon_{1}\right)$ e $\Upsilon_{1} v=w=-\frac{d^{2}}{d x^{2}} v$, assim $A_{0} \subset \Upsilon_{1}$. Usando Teorema 5.2.1, existe $\beta \in \mathbb{R}$ tal que $D\left(\Upsilon_{1}\right)=D\left(-\Delta_{\beta}\right)$. Agora mostramos que $\beta=-Z$. Seja $v \in D\left(\Upsilon_{1}\right) \operatorname{com} v(0) \neq 0$, então obtemos

$$
\left(\Upsilon_{1} v, v\right)=\left[v^{\prime}(0+)-v^{\prime}(0-)\right] v(0)+\int_{\mathbb{R}}\left|v_{x}\right|^{2} d x=\int_{\mathbb{R}}\left|v_{x}\right|^{2} d x+\beta[v(0)]^{2},
$$

o qual deveria ser igual a $\mathcal{Q}_{Z}^{1}(v, v)=\int_{\mathbb{R}}\left|v_{x}\right|^{2} d x-Z[v(0)]^{2}$, de onde $\beta=-Z$, como queríamos provar.

(III) O lema anterior nos permite identificar os operadores $\widetilde{\mathcal{L}}_{1}, \widetilde{\mathcal{L}}_{2}$ com os operadores $\mathcal{L}_{1, Z}, \mathcal{L}_{2, Z}$. O seguinte teorema verifica a analíticidade dos operadores $\mathcal{L}_{i, Z}$.

Lema 5.5.5. Como função de $Z,\left(\mathcal{L}_{1, Z}\right)$ e $\left(\mathcal{L}_{2, Z}\right)$ são famílias real-analíticas de operadores auto-adjuntos do tipo (B) no sentido de Kato.

Demonstração. Pelo Lema 5.5.4, Teorema VII-4.2 em Kato [35] e Reed\&Simon em [46] é suficiente mostrar que a família de formas bilineares $\left(\mathcal{Q}_{w, Z}^{1}\right)$ e $\left(\mathcal{Q}_{w, Z}^{2}\right)$ definidas em (5.63) são reais analíticas do tipo (B). De fato, o domínio das formas não depende de $Z$, isto é $H^{1}(\mathbb{R})$ para todo $Z \in \mathbb{R}$, basta provar então que elas são fechadas, limitadas inferiormente e para cada $v \in H^{1}(\mathbb{R})$ a função $Z \rightarrow$ $\mathcal{Q}_{w, Z}^{i}(v, v)$ é analítica. É imediato que elas são fechadas e limitadas inferiormente, a analiticidade da função $Z \rightarrow \mathcal{Q}_{w, Z}^{i}(v, v)$ segue da analiticidade da curva $Z \rightarrow$ $\phi_{w, Z} \in H^{1}(\mathbb{R}), \operatorname{com} \phi_{w, Z}$ dada em (5.45).

Observação 5.5.1. Notemos que para $-w>\frac{Z^{2}}{4}$ e $v, p \in H^{1}(\mathbb{R})$,

$$
\begin{aligned}
& \mathcal{Q}_{w, 0}^{1}(v, p)=\lim _{Z \rightarrow 0} \mathcal{Q}_{w, Z}^{1}(v, p)=\int_{\mathbb{R}} v_{x} p_{x} d x-\int_{\mathbb{R}}\left(w+3 \phi_{w, Z}^{2}+5 \phi_{w, Z}^{4}\right) v p d x \\
& \mathcal{Q}_{w, 0}^{2}(v, p)=\lim _{Z \rightarrow 0} \mathcal{Q}_{w, Z}^{2}(v, p)=\int_{\mathbb{R}} v_{x} p_{x} d x-\int_{\mathbb{R}}\left(w+\phi_{w, Z}^{2}+\phi_{w, Z}^{4}\right) v p d x
\end{aligned}
$$

onde $\mathcal{Q}_{w, 0}^{1}$ e $\mathcal{Q}_{w, 0}^{2}$, são as formas quadráticas associadas aos operadores definidos em (5.3). 
Pelo Teorema 5.1.1, sabemos que zero é um autovalor simples do operador $\mathcal{L}_{1,0}$ com autofunção associada $\frac{d}{d x} \phi_{w}$. A seguir veremos como dito autovalor é perturbado pelos operadores $\mathcal{L}_{1, Z}$.

Lema 5.5.6. Existe $Z_{0}>0$ e duas funções analíticas $\Pi:\left(-Z_{0}, Z_{0}\right) \rightarrow \mathbb{R} e$ $\Omega:\left(-Z_{0}, Z_{0}\right) \rightarrow L^{2}(\mathbb{R})$ tal que

(a) $\Pi(0)=0$ e $\Omega(0)=\frac{d}{d x} \phi_{w, 0}$.

(b) Para todo $Z \in\left(-Z_{0}, Z_{0}\right)$, $\Pi(Z)$ é o segundo autovalor de $\mathcal{L}_{1, Z}$ o qual é simples e $\Omega(Z)$ é a auto-função associada a $\Pi(Z)$.

(c) $Z_{0}$ pode ser escolhido de forma que o espectro do operador $\mathcal{L}_{1, Z}$ com $Z \in$ $\left(-Z_{0}, Z_{0}\right)$ é positivo exceto pelos dois primeiros autovalores.

Demonstração. Do Teorema 5.1.1 separamos o espectro $\sigma\left(\mathcal{L}_{1,0}\right)$ do operador $\mathcal{L}_{1,0}$ dado em (5.3) em duas partes $\sigma_{0}=\left\{\tau_{0}, 0\right\}$ e $\sigma_{1}$ por uma curva fechada $\Gamma$ (por exemplo um círculo) tal que $\sigma_{0}$ pertence ao interior da curva $\Gamma$ e $\sigma_{1}$ na parte exterior de $\Gamma$ (note que $\sigma_{1} \subset(a, \infty)$ para $a>0$ ). Usando o Lema 5.5.5 segue-se que $\mathcal{L}_{1, Z}$ converge a $\mathcal{L}_{1,0}$ quando $Z \rightarrow 0$ no sentido generalizado, logo do Teorema IV-3.16 em Kato [35] temos que $\Gamma \subset \rho\left(\mathcal{L}_{1, Z}\right)$ para $|Z|$ suficientemente pequeno e $\sigma\left(\mathcal{L}_{1, Z}\right)$ é separado por $\Gamma$ em duas partes tal que a parte de $\sigma\left(\mathcal{L}_{1, Z}\right)$ dentro de $\Gamma$ consiste de um número finito de autovalores com multiplicidade algébrica total dois (lembremos que zero não é um autovalor do operador $\mathcal{L}_{1, Z}$, para $Z \neq 0$ ). Consideramos para $\epsilon>0$ pequeno os conjuntos $\Gamma_{1}\left(\tau_{0}\right)=\left\{z \in \mathbb{C}|| z-\tau_{0} \mid<\epsilon\right\}$ e $\Gamma_{2}(0)=\{z \in \mathbb{C}|| z \mid<\epsilon\}$ tal que $\Gamma_{1}\left(\tau_{0}\right) \cap \Gamma_{2}(0)=\emptyset$ e os únicos pontos de $\sigma\left(\mathcal{L}_{1,0}\right)$ dentro do interior dos $\Gamma_{i}$ são $\tau_{0}$ e 0 . Como $\tau_{0}$ e 0 são simples temos então pelo Teorema de Kato-Rellich (ver Teorema XII.8 em Reed\&Simon [46]) a existência de duas funções analíticas $\Pi, \Omega$, definidas numa vizinhança de zero e satisfazendo os items (a),(b) e (c) acima. Isto finaliza a prova do lema.

Mostramos como o segundo autovalor perturbado $\Pi(Z)$ se movimenta conforme $Z$ muda de sinal. 
Lema 5.5.7. Existe $0<Z_{1}<Z_{0}$ tal que $\Pi(Z)<0$, para qualquer $Z \in\left(-Z_{1}, 0\right)$ e $\Pi(Z)>0$ para qualquer $Z \in\left(0, Z_{1}\right)$. Então para $Z$ negativo e pequeno $\mathcal{L}_{1, Z}$ tem exatamente dois autovalores negativos e para $Z$ positivo e pequeno $\mathcal{L}_{1, Z}$ tem exatamente um autovalor negativo.

Observação 5.5.2. Do Lema 5.5.7 vemos então que para $Z<0$ o autovalor 0 de $\mathcal{L}_{1,0}$ se movimenta para à esquerda, já para $Z>0$ este se movimenta para à direita.

Demonstração. Usando o Teorema de Taylor ao redor do ponto zero, temos que as funções $\Pi$ e $\Omega$ do Lema 5.5.6 podem ser escritas como

$$
\begin{aligned}
& \Pi(Z)=\beta Z+O\left(Z^{2}\right) \\
& \Omega(Z)=\phi_{w, 0}^{\prime}+Z \psi_{0}+O\left(Z^{2}\right),
\end{aligned}
$$

onde $\phi_{w, 0}^{\prime}=\frac{d}{d x} \phi_{w, 0}, \beta \in \mathbb{R}\left(\beta=\Pi^{\prime}(0)\right)$ e $\psi_{0} \in L^{2}(\mathbb{R})\left(\psi_{0}=\Omega^{\prime}(0)\right)$. Para obter o nosso resultado basta provar então que $\beta>0$. Assim, como a função $Z \rightarrow \phi_{w, Z}$ é analítica então para $Z$ perto de zero existe $\chi_{0} \in H^{1}(\mathbb{R})$ tal que

$$
\phi_{w, Z}=\phi_{w, 0}+Z \chi_{0}+O\left(Z^{2}\right) .
$$

Agora, da equação (5.37) temos que para todo $\psi \in H^{1}(\mathbb{R})$,

$$
\left\langle-\phi_{w, Z}^{\prime \prime}-w \phi_{w, Z}-\phi_{w, Z}^{3}-\phi_{w, Z}^{5}, \psi\right\rangle=Z \phi_{w, Z}(0) \psi(0)
$$

Logo, derivando (5.71) com respeito de $Z$ e avaliando em $Z=0$, obtemos

$$
\left\langle\mathcal{L}_{1,0} \chi_{0}, \psi\right\rangle=\phi_{w, 0}(0) \psi(0)
$$

Agora expandimos $\beta$ como função de $Z$. Vamos calcular a quantidade $\left\langle\mathcal{L}_{1, Z} \Omega(Z), \phi_{w, 0}^{\prime}\right\rangle$, de dois modos diferentes

(1) Como $\mathcal{L}_{1, Z} \Omega(Z)=\Pi(Z) \Omega(Z)$ entaõ segue de (5.69) que

$$
\left\langle\mathcal{L}_{1, Z} \Omega(Z), \phi_{w, 0}^{\prime}\right\rangle=\beta Z\left\|\phi_{w, 0}^{\prime}\right\|^{2}+O\left(Z^{2}\right)
$$


(2) Como $\mathcal{L}_{1, Z}$ é auto-adjunto e $\phi_{w, 0}^{\prime} \in D\left(\mathcal{L}_{1, Z}\right)$, então $\left\langle\mathcal{L}_{1, Z} \Omega(Z), \phi_{w, 0}^{\prime}\right\rangle=$ $\left\langle\Omega(Z), \mathcal{L}_{1, Z} \phi_{w, 0}^{\prime}\right\rangle$. De (5.70) deduzimos que

$$
\begin{aligned}
\mathcal{L}_{1, Z} \phi_{w, 0}^{\prime} & =\mathcal{L}_{1,0}\left(\phi_{w, 0}^{\prime}\right)+3\left(\phi_{w, 0}^{2}-\phi_{w, Z}^{2}\right) \phi_{w, 0}^{\prime}+5\left(\phi_{w, 0}^{4}-\phi_{w, Z}^{4}\right) \phi_{w, 0}^{\prime} \\
& =3\left(\phi_{w, 0}^{2}-\phi_{w, Z}^{2}\right) \phi_{w, 0}^{\prime}+5\left(\phi_{w, 0}^{4}-\phi_{w, Z}^{4}\right) \phi_{w, 0}^{\prime} \\
& =-6 Z \phi_{w, 0} \phi_{w, 0}^{\prime} \chi_{0}-20 Z \phi_{w, 0}^{3} \phi_{w, 0}^{\prime} \chi_{0}+O\left(Z^{2}\right)
\end{aligned}
$$

logo de (5.69) e (5.74) obtemos que

$$
\begin{aligned}
\left\langle\mathcal{L}_{1, Z} \Omega(Z), \phi_{w, 0}^{\prime}\right\rangle & =-6 Z\left\langle\phi_{w, 0}^{\prime}, \chi_{0} \phi_{w, 0} \phi_{w, 0}^{\prime}\right\rangle \\
& -20 Z\left\langle\phi_{w, 0}^{\prime}, \chi_{0} \phi_{w, 0}^{3} \phi_{w, 0}^{\prime}\right\rangle+O\left(Z^{2}\right) .
\end{aligned}
$$

Por outro lado observamos que

$$
\mathcal{L}_{1,0}\left(-w \phi_{w, 0}-\phi_{w, 0}^{3}-\phi_{w, 0}^{5}\right)=6 \phi_{w, 0}\left(\phi_{w, 0}^{\prime}\right)^{2}+20 \phi_{w, 0}^{3}\left(\phi_{w, 0}^{\prime}\right)^{2}
$$

portanto de (5.72), (5.75) e (5.76), deduzimos que

$$
\begin{aligned}
\left\langle\mathcal{L}_{1, Z} \Omega(Z), \phi_{w, 0}^{\prime}\right\rangle & =-Z\left\langle\chi_{0}, 6 \phi_{w, 0}\left(\phi_{w, 0}^{\prime}\right)^{2}+20 \phi_{w, 0}^{3}\left(\phi_{w, 0}^{\prime}\right)^{2}\right\rangle \\
& =-Z\left\langle\mathcal{L}_{1,0} \chi_{0},-w \phi_{w, 0}-\phi_{w, 0}^{3}-\phi_{w, 0}^{5}\right\rangle \\
& =-Z \phi_{w, 0}(0)\left(-w \phi_{w, 0}(0)-\phi_{w, 0}^{3}(0)-\phi_{w, 0}^{5}(0)\right)+O\left(Z^{2}\right) .
\end{aligned}
$$

(3) Em fim de (5.73) e (5.77) temos que

$$
\beta=\frac{w \phi_{w, 0}^{2}(0)+\phi_{w, 0}^{4}(0)+\phi_{w, 0}^{6}(0)}{\left\|\phi_{w, 0}^{\prime}\right\|^{2}}+O(Z) .
$$

Por outro lado, não é difícil ver de (5.6) que

$$
\phi_{w, 0}(0)=\sqrt{\frac{-3+\sqrt{9-48 w}}{4}},
$$

e que o polinômio $q(x)=w x^{2}+x^{4}+x^{6}$ é positivo se $x>r_{0}=\sqrt{\frac{-1+\sqrt{1-4 w}}{2}}$. Assim como $\phi_{w, 0}(0)>r_{0}$ deduzimos então que $\Pi^{\prime}[0]=\beta>0$, como queríamos provar. 
Observação 5.5.3. A prova do Lema 5.5.7 mostra de fato que a função $Z \rightarrow$ $\Pi(Z)$ é estritamente crescente numa vizinhança de zero.

(IV) Agora estamos em condições de contar o número de autovalores negativos do operador $\mathcal{L}_{1, Z}$ para todo $Z$. Usaremos um argumento clássico de continuação baseado nas projeções de Riesz. O número de autovalores negativos de $\mathcal{L}_{1, Z}$ será denotado por $n\left(\mathcal{L}_{1, Z}\right)$.

Lema 5.5.8. Seja $w<0$ tal que $-w>\frac{Z^{2}}{4}$. Então

(1) $\operatorname{Para} Z \geq 0, n\left(\mathcal{L}_{1, Z}\right)=1$,

(2) $\operatorname{Para} Z<0, n\left(\mathcal{L}_{1, Z}\right)=2$.

Demonstração. Seja $Z<0$ e definamos $Z_{\infty}$ por

$$
\begin{array}{r}
Z_{\infty}=\inf \left\{z<0 \mid \mathcal{L}_{1, Z}\right. \text { tem exatamente dois autovalores } \\
\text { negativos para todo } Z \in(z, 0)\} .
\end{array}
$$

Do Lema 5.5.7 temos que $\mathcal{L}_{1, Z}$ tem exatamente dois autovalores negativos para todo $Z \in\left(Z_{1}, 0\right)$, $\log$ o $Z_{\infty}$ é bem definido e $Z_{\infty} \in[-\infty, 0)$. Vamos mostrar que $Z_{\infty}=-\infty$. Suponhamos o cóntrario, isto é, suponhamos que $Z_{\infty}>-\infty$. Seja $N=n\left(\mathcal{L}_{1, Z_{\infty}}\right)$ e $\Gamma$ uma curva fechada (por exemplo un retângulo ou um círculo) tal que $0 \in \Gamma \subset \rho\left(\mathcal{L}_{1, Z_{\infty}}\right)$ e tal que todo o espectro negativo de $\mathcal{L}_{1, Z_{\infty}}$ pertençã ao interior da curva $\Gamma$. Usando o Lema 5.5.5 temos que $\mathcal{L}_{1, Z} \rightarrow \mathcal{L}_{1, Z_{\infty}}$ quando $Z \rightarrow Z_{\infty}$ no sentido generalizado, logo existe $\delta>0$ tal que para todo $Z \in\left[Z_{\infty}-\delta, Z_{\infty}+\delta\right]$ temos que $\Gamma \subset \rho\left(\mathcal{L}_{1, Z}\right)$ e $N=n\left(\mathcal{L}_{1, Z}\right)$. Lembramos aqui que esta conclusão é deduzida da existência de uma família analítica de projeções de Riesz $Z \rightarrow P(Z)$ sobre a parte negativa do espectro dos operadores $\mathcal{L}_{1, Z}$ contida dentro da curva $\Gamma$, ou seja, para

$$
P(Z)=-\frac{1}{2 \pi i} \int_{\Gamma}\left(\mathcal{L}_{1, Z}-\xi\right)^{-1} d \xi
$$


implica que

$$
\operatorname{dim}(\operatorname{Rank} P(Z))=\operatorname{dim}\left(\operatorname{Rank} P\left(Z_{\infty}\right)\right)=N, \forall Z \in\left[Z_{\infty}-\delta, Z_{\infty}+\delta\right] .
$$

Agora, pela definição de $Z_{\infty}$, existe $z_{0}$ tal que $Z_{\infty}<z_{0}<Z_{\infty}+\delta$ e $\mathcal{L}_{1, Z}$ tem exatamente dois autovalores negativos para todo $Z \in\left(z_{0}, 0\right)$. Então $\mathcal{L}_{1, Z_{\infty}+\delta}$ tem dois autovalores negativos e do Lema 5.5.7 obtemos que $N=2$ e portanto $\mathcal{L}_{1, Z}$ tem dois autovalores negativos para $Z \in\left(Z_{\infty}-\delta, 0\right)$ o que contradiz a definição de $Z_{\infty}$. Assim, $Z_{\infty}=-\infty$. Um análise similar pode ser aplicado ao caso $Z>0$. Isto finaliza a prova do lema.

Observação 5.5.4. No lema anterior, é possivel escolher $\Gamma$ independentemente do parâmetro $Z$. De fato, consideremos $Z<0$. Então, para todo $Z$ temos que $\phi_{w, Z}(x) \leq \phi_{w, 0}(0)=: \alpha$, com $\phi_{w, 0}(0)$ dado em (5.79). Logo para $\|f\|=1 e$ $f \in D$

$$
\left\langle\mathcal{L}_{1, Z} f, f\right\rangle \geq-3 \int \phi_{w, Z}^{2} f^{2} d x-5 \int \phi_{w, Z}^{4} f^{2} d x \geq-3 \alpha^{2}-5 \alpha^{4}
$$

Então, $\inf \sigma\left(\mathcal{L}_{1, Z}\right) \geq-3 \alpha^{2}-5 \alpha^{4}$ para todo $Z<0$. Portanto $\Gamma$ pode ser escolhido como o retângulo $\Gamma=\partial R$, onde $R$ é dado por

$$
\begin{gathered}
R=\left\{z \in \mathbb{C} \mid z=z_{1}+i z_{2},\left(z_{1}, z_{2}\right) \in\left[-3 \alpha^{2}-5 \alpha^{4}-1,0\right] \times[-d, d],\right. \\
\text { para algum } d>0\} .
\end{gathered}
$$

Lema 5.5.9. A função $Z \rightarrow \Omega(Z)$ definida no Lema 5.5.6 e associada ao segundo autovalor do operador $\mathcal{L}_{1, Z}$ pode ser estendida ao intervalo $(-\infty, \infty)$. Além disso, $\Omega(Z) \in H^{1}(\mathbb{R})$ é uma função ímpar para todo $Z \in(-\infty, \infty)$.

Demonstração. Do Lema 5.5.5 e o Teorema XII.7 em [46] o conjunto $\Gamma_{0}=$ $\left\{(Z, \lambda) \mid Z \in \mathbb{R}, \lambda \in \rho\left(\mathcal{L}_{1, Z}\right)\right\}$ é aberto e

$$
(Z, \lambda) \in \Gamma_{0} \rightarrow\left(\mathcal{L}_{1, Z}-\lambda\right)^{-1}
$$

é uma função analítica nas duas variáveis. Então, podemos repetir o argumento do Lema 5.5.6 em cada ponto $Z$ e em cada vizinhança de $Z$ para obter funções 
$\Omega(Z)$ e $\Pi(Z)$ são analíticas para todo $Z \in \mathbb{R}$. Agora consideramos o caso $Z<0$ (o caso $Z>0$ é análogo). Sabemos dos Lemas 5.5.2 e 5.5.6 que as autofunções $\Omega(Z)$ são pares ou ímpares e $\Omega(0)=\frac{d}{d x} \phi_{w, 0}$ é ímpar. Então da igualdade

$$
\lim _{Z \rightarrow 0}\langle\Omega(Z), \Omega(0)\rangle=\|\Omega(0)\|^{2} \neq 0
$$

obtemos que $\langle\Omega(Z), \Omega(0)\rangle \neq 0$ para $Z$ perto de 0 . Logo $\Omega(Z)$ é ímpar para $Z$ perto de 0. Agora, seja $z_{\infty}$ definido por

$$
z_{\infty}=\inf \{z<0 \mid \Omega(Z) \text { é ímpar } \forall Z \in(z, 0]\}
$$

Suponhamos que $z_{\infty}>-\infty$. Se $\Omega\left(z_{\infty}\right)$ é ímpar, então por continuidade existe $\delta>0$ tal que $\Omega\left(z_{\infty}-\delta\right)$ é ímpar, isto contradiz a escolha de $z_{\infty}$. Portanto o Lema 5.5.2 implica que $\Omega\left(z_{\infty}\right)$ é par. Por outro lado $\Omega\left(z_{\infty}\right)$ é o limite de funções ímpares portanto $\Omega\left(z_{\infty}\right)$ é ímpar. Assim deduzimos que $\Omega\left(z_{\infty}\right) \equiv 0$, o qual é uma contradição pois $\Omega\left(z_{\infty}\right)$ é uma autofunção. Isto conclue a prova do lema.

\subsubsection{Condição de convexidade}

Nesta subseção vamos estudar o crescimento-decrescimento da função $w \rightarrow\left\|\phi_{w, Z}\right\|^{2}$, com $-w>\frac{Z^{2}}{4}$. Como veremos nossos cálculos serão de tipo numérico, pois as fórmulas que obtemos resultam ser muito complicadas para ser analizadas analiticamente. Note que de (5.45) temos

$$
\begin{aligned}
\int_{-\infty}^{\infty} \phi_{w, Z}^{2}(x) d x & =\int_{-\infty}^{\infty} \frac{1}{\alpha(w)+\beta(w) \cosh (2 \sqrt{-w}(|x|+b))} d x \\
& =\frac{1}{\sqrt{-w}} \int_{2 \sqrt{-w} b}^{\infty} \frac{1}{\alpha(w)+\beta(w) \cosh (u)} d u
\end{aligned}
$$

$\operatorname{com} \alpha(w)=\frac{-1}{4 w}, \beta(w)=\frac{\sqrt{9-48 w}}{-12 w}$ e $b=R_{w}^{-1}\left(\frac{Z}{2 \sqrt{-w}}\right)$ aqui $R_{w}$ é dado por

$$
R_{w}(b)=\frac{\beta(w) \operatorname{senh}(2 \sqrt{-w} b)}{\alpha(w)+\beta(w) \cosh (2 \sqrt{-w} b)},
$$


(ver equações $(5.43)$ e $(5.45))$. Fazendo $\gamma(w)=\frac{\sqrt{3}}{\sqrt{3-16 w}}$ podemos reescrever (5.82) como

$$
\begin{aligned}
\int_{-\infty}^{\infty} \phi_{w, Z}^{2}(x) d x & =\frac{1}{\beta(w) \sqrt{-w}} \int_{2 \sqrt{-w} b}^{\infty} \frac{1}{\gamma(w)+\cosh (u)} d u \\
& =-\left.2 \sqrt{3} \operatorname{arctg}\left(\frac{\sqrt{3}-\sqrt{3-16 w}}{4 \sqrt{-w}} \operatorname{tagh}\left(\frac{u}{2}\right)\right)\right|_{u=2 \sqrt{-w} b} ^{u=\infty}
\end{aligned}
$$

isto é

$$
\left\|\phi_{w, Z}\right\|^{2}=-2 \sqrt{3}[\operatorname{arctg}(\theta(w))-\operatorname{arctg}(\theta(w) \operatorname{tagh}(\sqrt{-w} b))],
$$

$\operatorname{com} \theta(w)=\frac{\sqrt{3}-\sqrt{3-16 w}}{4 \sqrt{-w}}$. Agora é claro que para obter uma expressão para $\partial_{w}\left\|\phi_{w, Z}\right\|^{2}$ precisamos achar uma fórmula que nos permita calcular $\frac{d}{d w} b$, assim da relação (5.83) temos que para

$$
H(w, b)=2 \sqrt{-w} R_{w}(b)
$$

de (5.43) segue que $H(w, b)=Z$. Agora, como $H_{b}>0$ então aplicando o Teorema da função implícita deduzimos que

$$
\frac{d b}{d w}=-\frac{H_{w}}{H_{b}},
$$

assim de (5.83) e (5.86), deduzimos que $b^{\prime}(w)=\frac{d b}{d w}$ é dado por,

$$
\frac{d b}{d w}(w, b)=\frac{4 \sqrt{3} \sqrt{-w} h(w) b \cosh (s(w) b)+2 \sqrt{3}(3-32 w) \operatorname{senh}(s(w) b)+t(w)}{8(-w)^{\frac{3}{2}} \sqrt{h(w)}(h(w)+\sqrt{3} \sqrt{h(w)} \cosh (s(w) b))},
$$

$\operatorname{com} t(w)=h(w)^{\frac{3}{2}}(2 s(w) b+\operatorname{senh}(2 s(w) b)), h(w)=3-16 w$ e $s(w)=2 \sqrt{-w}$. Istos calculos mostram que é possível obter uma fórmula para analisar $\partial_{w}\left\|\phi_{w, Z}\right\|^{2}$. Assim, de (5.85) obtemos

$$
\begin{aligned}
& \frac{1}{2 \sqrt{3}} \partial_{w}\left\|\phi_{w, Z}\right\|^{2}=\frac{3-\sqrt{3 h(w)}}{u(w) \sqrt{-w h(w)}}+ \\
& \frac{\operatorname{sech}^{2}(\sqrt{-w} b)\left[-2 b u(w) \sqrt{-w}+\operatorname{senh}(2 \sqrt{-w} b)(3-\sqrt{3 h(w)})+4 u(w) b^{\prime}(w)(-w)^{\frac{3}{2}}\right]}{2 \sqrt{-w h(w)}\left(8 w+\tanh ^{2}(\sqrt{-w} b)(-3+8 w+\sqrt{3 h(w)})\right)},
\end{aligned}
$$

com $u(w)=h(w)+\sqrt{3 h(w)}$. Usando o programa Mathematica obtemos os seguintes gráficos para as funções $(w, Z) \rightarrow-\left\|\phi_{w, Z}\right\|^{2}$ e $(w, Z) \rightarrow-\partial_{w}\left\|\phi_{w, Z}\right\|^{2}$ dadas em (5.85) e (5.88) respectivamente. Lembramos aqui que $(w, Z)$ devem satizfazer a relação $\frac{Z^{2}}{4}<$ $-w$. Começamos com alguns gráficos da função $(w, Z) \rightarrow-\left\|\phi_{w, Z}\right\|^{2}$, 


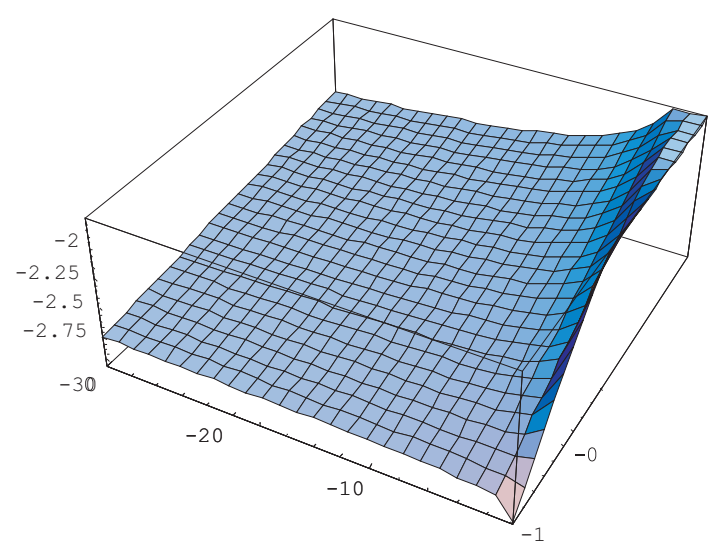

com $w \in(-30,-0.25), Z \in(-1,1)$.

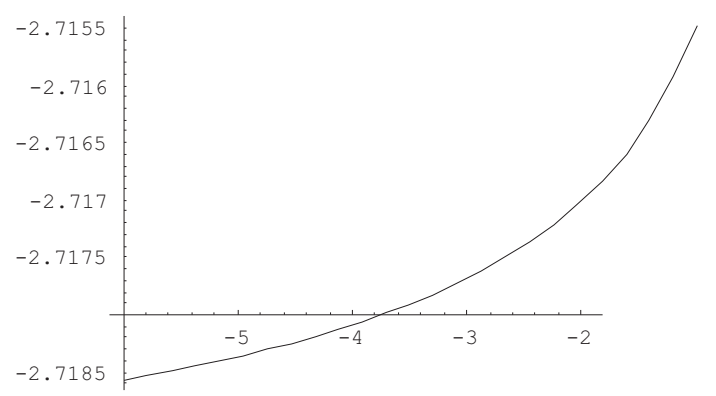

$\operatorname{com} w \in(-6,-1), Z=-0.86$.

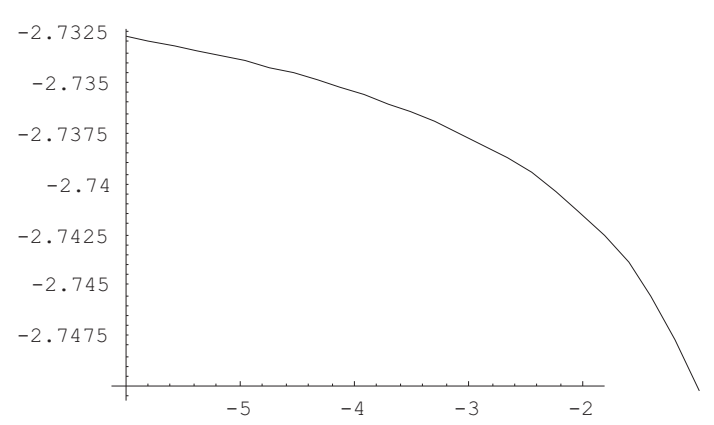

com $w \in(-6,-1), Z=-0.9$.

Uma análise mais detalhado dos gráficos nos mostram uma mudança do crescimento da função $(w, Z) \rightarrow-\left\|\phi_{w, Z}\right\|^{2}$ para algum valor do parâmetro $Z \in(-0.9,-0.8)$. Agora, mostramos o comportamento da função $(w, Z) \rightarrow-\partial_{w}\left\|\phi_{w, Z}\right\|^{2}$ para diferentes valores dos parâmetros $w$ e $Z$, particular interesse damos aos valores da função no intervalo $Z \in(-0.9,-0.8)$. Assim, 


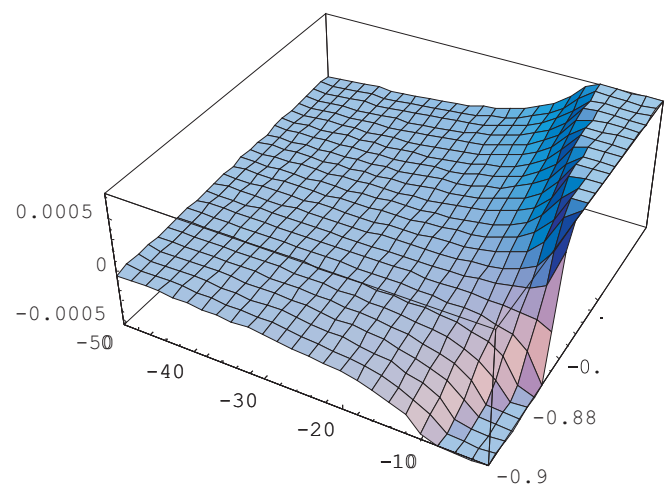

para $w \in(-50,-2), Z \in(-0.9,-0.8)$.

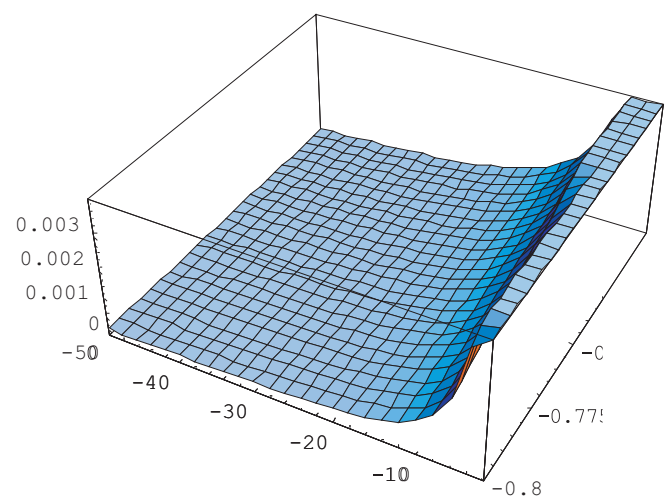

para $w \in(-50,-2), Z \in(-0.8,-0.7)$.

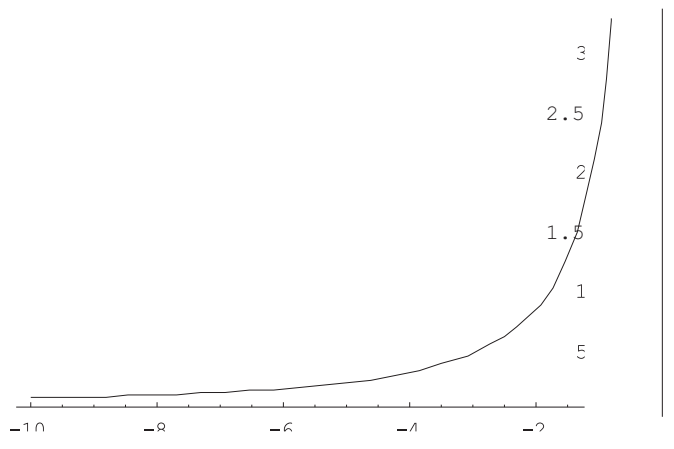

para $w \in(-10,-0.8), Z=-0.86602$. 


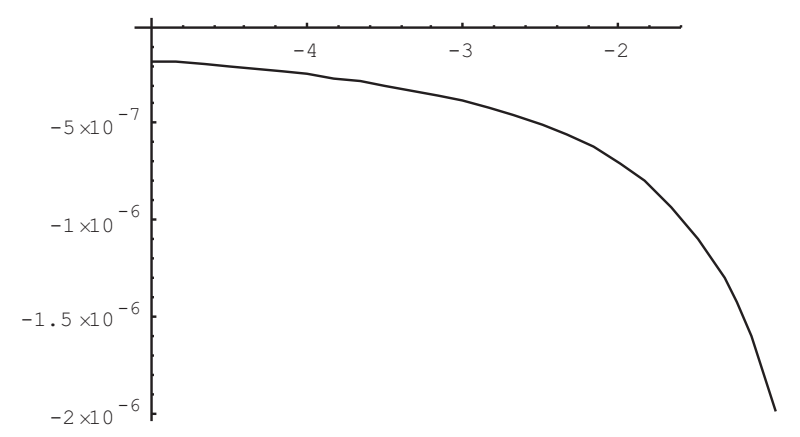

para $w \in(-5,-1), Z=-0.86603$.

Assim, dos nossos cálculos numéricos podemos estabelecer o seguinte teorema que resume o análise do crescimento da função $(w, Z) \rightarrow-\left\|\phi_{w, Z}\right\|^{2}$ feito nesta subseção,

Teorema 5.5.1. Sejam $w<0, Z \in \mathbb{R}$ satisfazendo $\frac{Z^{2}}{4}<-w$. Então para $Z^{*} \approx$ -0.866025403784 , a função $(w, Z) \rightarrow-\left\|\phi_{w, Z}\right\|^{2}$ satisfaz as seguintes propriedades de crescimento

$$
\left\{\begin{array}{l}
-\partial_{w}\left\|\phi_{w, Z}\right\|^{2}>0, \quad \text { se } Z>Z^{*}, \\
-\partial_{w}\left\|\phi_{w, Z}\right\|^{2}<0, \quad \text { se } Z<Z^{*} .
\end{array}\right.
$$

Observação 5.5.5. O valor exato para $Z^{*}$ é obtido fazendo o lado direito da equação em (5.88) igual a zero.

\subsubsection{Resultados de Estabilidade}

Agora, estamos em condições de estabelecer os nossos resultados de estabilidadeinstabilidade associados com a órbita $\Omega_{\phi_{w, Z}}$ definida em (5.5) gerada pelas soluções $\phi_{w, Z}$ dadas em (5.45). O critério para obter estabilidade ou instabilidade em nosso caso é uma aplicação da teoria abstrata de Grillakis\&Shatah\&Straus em [30]. Desta forma, consideremos o operador linear $H_{w, Z}$ definido em (5.47) e denotemos por $n\left(H_{w, Z}\right)$ o número de autovalores negativos de $H_{w, Z}$. Definamos

$$
p_{Z}\left(w_{0}\right)= \begin{cases}1, & \text { se }-\partial_{w}\left\|\phi_{w, Z}\right\|^{2}>0 \text { em } w=w_{0} \\ 0, & \text { se }-\partial_{w}\left\|\phi_{w, Z}\right\|^{2}<0 \text { em } w=w_{0} .\end{cases}
$$


Agora, como a equação (5.2) não é invariante por translações, temos que o núcleo do operador $H_{w, Z}$ é gerado pelo vetor $\left[0, \phi_{w, Z}\right]=:\left[0, a_{w}\right]$ (na notação do Apêndice A). Assim, seguindo as idéias na prova do teorema de estabilidade no Apêndice A, junto com as hipóteses 1 e 3 da página 106 abaixo e do teorema de instabilidade em [30], temos o seguinte resultado.

Teorema 5.5.2. Seja $-w_{0}>\frac{Z^{2}}{4}$.

(1) A standing wave $e^{-i w_{0} t} \phi_{w_{0}, Z}$ é estável em $H^{1}(\mathbb{R})$ se $n\left(H_{w_{0}, Z}\right)=p_{Z}\left(w_{0}\right)$.

(2) A standing wave $e^{-i w_{0} t} \phi_{w_{0}, Z}$ é instável em $H^{1}(\mathbb{R})$ se $n\left(H_{w_{0}, Z}\right)-p_{Z}\left(w_{0}\right)$ é ímpar.

Com isto temos os seguintes resultados sobre estabilidade para a CQD-S.

Teorema 5.5.3. Seja $-w>\frac{Z^{2}}{4}$, então temos

I- Para $Z \geq 0$, a órbita $\Omega_{\phi_{w, Z}}$ gerada por $\phi_{w, Z}$ em (5.45), é estável pelo fluxo da equação $C Q D-S$ no espaço $H^{1}(\mathbb{R})$.

II- Para $Z \in\left(Z^{*}, 0\right)$, a órbita $\Omega_{\phi_{w, Z}}$ gerada por $\phi_{w, Z}$ em (5.45), é instável pelo fluxo da equação $C Q D-S$ no espaço $H^{1}(\mathbb{R})$.

III- Para $Z \in\left(Z^{*}, \infty\right)$, a órbita $\Omega_{\phi_{w, Z}}$ gerada por $\phi_{w, Z}$ em (5.45), é estável pelo fluxo da equação $C Q D$-S no espaço $H_{\text {even }}^{1}(\mathbb{R})$.

$I V$ - Para $Z<Z^{*}$, a órbita $\Omega_{\phi_{w, Z}}$ gerada por $\phi_{w, Z}$ em (5.45), é instável pelo fluxo da equação $C Q D-S$ no espaço $H_{\text {even }}^{1}(\mathbb{R})$.

Demonstração. Do Teorema 5.5.1 temos que para $-w>\frac{Z^{2}}{4}$ e $Z>Z^{*}, p_{Z}(w)=1$. Por outro lado, do Lema 5.5.1 temos que 0 é um autovalor simples do operador $\mathcal{L}_{2, Z}$ e do Lema 5.1.1 temos que $\mathcal{L}_{1, Z}$ tem núcleo trivial. Logo do Teorema 5.5.2 e do Lema 5.5.8 deduzimos os items $I$ e $I I$ do teorema.

Agora, do Lema 5.5.9 sabemos que o segundo autovalor do operador $\mathcal{L}_{1, Z}$ considerado no espaço $L^{2}(\mathbb{R})$ é associado com uma autofunção ímpar. Portanto dito autovalor desaparece quando o problema é considerado no espaço $H_{\text {even }}^{1}(\mathbb{R})$. Além disso, como 
$\phi_{w, Z}$ é uma função par e satisfaz a relação $\left\langle\mathcal{L}_{1, Z} \phi_{w, Z}, \phi_{w, Z}\right\rangle<0$, para todo $Z \in \mathbb{R}$, então obtemos que o primeiro autovalor do operador $\mathcal{L}_{1, Z}$ ainda está presente quando o problema é restrito ao espaço das funções pares de $H^{1}(\mathbb{R}), H_{\text {even }}^{1}(\mathbb{R})$. Logo deduzimos que $n\left(\left.H_{w, Z}\right|_{H_{\text {even }}^{1}(\mathbb{R})}\right)=1$ e portanto os items $I I I$ e $I V$ seguem do Teorema 5.5.2 trocando $H^{1}(\mathbb{R})$ por $H_{\text {even }}^{1}(\mathbb{R})$ e do Teorema 5.3.1. Isto finaliza a prova.

Nota 5.5.1. O critério no Teorema 5.5.2, não diz nada da estabilidade da órbita $\Omega_{\phi_{w, Z}}$ no espaço $H^{1}(\mathbb{R})$ para $Z<Z^{*}$, pois $n\left(\left.H_{w, Z}\right|_{H_{\text {even }}^{1}(\mathbb{R})}\right)=2$ e $p_{Z}(w)=0$.

Como não temos um resultado de boa colocação global em $H^{1}(\mathbb{R})$ para a Eq CQD$\mathrm{S}$ (ver Teorema 5.3.2). Os resultados de estabilidade orbital para a órbita $\Omega_{\phi_{w, Z}}$ (ver 5.5.1) no Teorema 5.5.3, são apenas válidos para os valores de $t$ onde a solução $u(t)$ existe.

Porém, dito resultado junto com o teorema de boa colocação local para a CQD-S (ver Teorema 5.3.1), implicam a existência global de soluções quando o dado inicial está perto da órbita. De fato, temos o seguinte teorema:

Teorema 5.5.4. (Existência global de soluções próximas da órbita) Toda solução u da equação $C Q D-S$, com dado inicial $u(0)=f$ próximas da órbita $\Omega_{\phi_{w, Z}}$, é globalmente bem definida.

Demonstração. Claramente, para todo $s \in \mathbb{R}$ temos

$$
\begin{aligned}
\|u(t)\|_{1} & \leq\left\|u(t)-e^{-i s} \phi_{w, Z}\right\|_{1}+\left\|e^{-i s} \phi_{w, Z}\right\|_{1} \\
& =\left\|u(t)-e^{-i s} \phi_{w, Z}\right\|_{1}+\left\|\phi_{w, Z}\right\|_{1} .
\end{aligned}
$$

Assim de (5.91) e da estabilidade da órbita $\Omega_{\phi_{w, Z}}$, temos que, para $\epsilon>0$ existe $\delta>0$ tal que

$$
\|u(t)\|_{1} \leq \epsilon+\left\|\phi_{w, Z}\right\|_{1}, \quad \forall t \in\left[0, T^{*}(f)\right),
$$

sempre que $\inf _{s \in[0,2 \pi)}\left\|f-e^{-i s} \phi_{w, Z}\right\|_{1}<\delta$, aqui $T^{*}(f)$ denota o tempo maximal de existência da solução $u$ dado pelo Teorema 5.3.1.

Em particular, (5.92) implica a limitação da solução u. Isto junto com o Teorema 4.1.4 prova o teorema. 
Assim, o Teorema 5.5.4, nos permite obter os resultados de estabilidade do Teorema 5.5.3 para todo $t \in \mathbb{R}$, pois a solução $u(t)$ é globalmente definida. 


\section{CAPÍTULO 6}

Neste capítulo, nosso principal objetivo será apresentar algumas idéias dos problemas que podem ser abordados num futuro próximo usando possivelmente as ferramentas desenvolvidas ao longo deste trabalho.

1. Um dos modelos dispersivos no qual estamos interessados em estudar é a equação não-linear de tipo Schrödinger:

$$
i u_{t}+u_{x x}+\lambda|u|^{2} u+\mu|u|^{4} u=0
$$

com $\lambda, \mu$ parâmetros reais, $x, t \in \mathbb{R}$ e $u: \mathbb{R} \times \mathbb{R} \rightarrow \mathbb{C}$. Notamos que quando $\lambda=$ $\mu=1$, obtemos exatamente a equação CQ-S estudada no capítulo 4. Portanto todas as questões estudadas para a equação CQ-S no capítulo 4 podem chegar a ser estudadas para a Eq (6.1).

É conhecido que a mudança nos sinais dos parâmetros $\lambda, \mu$, produz diferentes comportamentos na dinâmica da equação (6.1). Por exemplo, quando $\mu \leq 0$ é possivel obter um resultado de boa colocação global no espaço energia $H^{1}$ (periódico ou não periódico). Porém, no caso em que $\mu>0$ dito resultado é só possivel quando a norma $L^{2}$ do dado inicial é pequena (ver seção 4.1). Este tipo de fenômeno nos mostra as complicações que aparecem no comportamento da 
dinâmica da Eq. (6.1) conforme os parâmetros $\lambda, \mu$ são escolhidos. Assim, uma das primeiras perguntas que poderia ser respondidas tem a ver com a forma em que as standing waves associadas com a equação (6.1) mudam dependendo da escolha dos parâmetros $\lambda, \mu$. Isto é, mudança nas soluções do tipo

$$
u(x, t)=e^{-i w t} a_{w}(x),
$$

$\operatorname{com} w \in \mathbb{R}$ e $a_{w}: \mathbb{R} \rightarrow \mathbb{R}$ uma função periódica.

Para entender os diferentes tipos de standing waves que a equação (6.1) admite, substituimos (6.2) em (6.1) e obtemos facilmente que o perfil da onda $a_{w}=a$ satisfaz

$$
a^{\prime \prime}+w a+\lambda a^{3}+\mu a^{5}=0
$$

assim, no apêndice B vemos como a equação (6.3) admite diferentes tipos de soluções dependendo do sinal dos parâmetros $w, \lambda$ e $\mu$. O tipo de onda periódica pode ser classificada dependendo do sinal de seu perfil. Assim, temos ondas positivas, negativas ou simplesmente ondas que mudam de sinal.

De acordo ao análise qualitativo feito no apêndice B sobre a equação differencial (6.3), existem três casos interessantes que tem perfil periódico positivo, além daquele estudado no capítulo 4. à saber,

(a) $\lambda^{2}>4 w \mu$, com $w>0, \lambda<0, \mu>0$. Ver a segunda figura do item III-(a)-i, no apêndice B.

(b) $\lambda^{2}>4 w \mu$, com $w<0, \lambda>0, \mu<0$. Ver a segunda figura do item III-(a)-ii, no apêndice B. Neste mesmo caso observamos a existência de uma onda solitaria e positiva, ver a primeira figura do item III-(a)-ii, no apêndice B.

(c) $w<0, \lambda<0, \mu>0$. Ver o item III-(b)-iv, do apêndice B.

Agora, o caso em que o perfil da onda $a_{w}$ é periódico e muda de sinal aparece com maior frequência (ver apêndice B). Destacamos aqui os seguintes casos: 
(a) $\lambda^{2}>4 w \mu$, com $w>0, \lambda<0, \mu>0$. Ver a segunda figura do item III-(a)-i, no apêndice $B$, onde aparecem dois tipos de soluções periódicas que mudam de sinal.

(b) $\lambda^{2}>4 w \mu$, com $w<0, \lambda>0, \mu<0$. Ver a segunda figura do item III-(a)-ii, no apêndice $B$, onde aparece um tipo de solução periódica.

(c) $w>0, \lambda>0, \mu<0$. Ver a figura do item III-(b)-ii, no apêndice B.

(d) $w<0, \lambda>0, \mu>0$. Ver a figura do item III-(b)-iii, no apêndice B.

Por outro lado, uma pergunta mais delicada de responder é a estabilidade orbital de ditas ondas. Nesta parte, um análise espectral do operador

$$
\mathcal{L}_{1,0}=-\frac{d^{2}}{d x^{2}}-w-3 \lambda a_{w}^{2}-5 \mu a_{w}^{4},
$$

é fundamental. Neste caso, para este análise, esperamos usar de maneira eficaz o recente trabalho realizado por A. Neves em [42] onde ele apresenta uma nova versão do Teorema de Floquet (ver [39]).

2. Inspirados nos cálculos feitos no Capítulo 5, esperamos obter uma teoria geral de existência e estabilidade, para o modelo

$$
i u_{t}+u_{x x}+Z \delta(x) u+\lambda u|u|^{2}+\mu u|u|^{4}=0, \quad Z \in \mathbb{R}
$$

onde $\delta$ é a medida de Dirac centrada no ponto $x=0, \lambda, \mu$ parâmetros reais. Neste caso o perfil $a_{w, Z}:=a$, satisfaz,

$$
a^{\prime \prime}+w a+Z \delta(x) a+\lambda a^{3}+\mu a^{5}=0 .
$$

Logo, levando em consideração o análise feito no Apêndice B, dois casos são de particular interesse para o nosso trabalho posterior:

(a) $\lambda^{2}>4 w \mu$, com $w<0, \lambda>0, \mu<0$. Ver a primeira figura do item III-(a)-ii, no apêndice B.

(b) $w<0, \lambda<0, \mu>0$. Ver o item III-(b)-iv, no apêndice B. 
Já para o análise espectral do operador

$$
\mathcal{L}_{1, Z} g=-\frac{d^{2}}{d x^{2}} g-w g-3 \lambda a_{w, Z}^{2} g-5 \mu a_{w, Z}^{4} g,
$$

com domínio $D$ em (5.38).

3. Outro problema que pode ser abordado é a existência e estabilidade de standing waves para a equação (6.5) quando o perfil da onda é de tipo periódico. Em particular pode ser abordado o caso quando $\lambda=1$ e $\mu=1$ na equação (6.5). Aqui a informação espectral obtida na seção 4.3, assim como as idéias desenvolvidas no capítulo 5 serão de extrema importância para obter um resultado de estabilidade orbital (ver [11]).

\section{Existência e estabilidade de soluções de equilibrio}

Outro tipo de modelos que podem ser estudados usando as ferramentas aplicadas nesta tese, são

$$
u_{t}=u_{x x}+w u+P(u)
$$

$\operatorname{com}(x, t) \in \mathbb{R} \times \mathbb{R}^{+}, u(x, t) \in \mathbb{R}, w \in \mathbb{R}$ um parâmetro e $P$ um polinômio com coeficientes reais de grau maior a um.

O problema da existência de soluções de equilíbrio de tipo periódico, isto é soluções para a Eq. (6.8) do tipo $u(x, t)=a(x)$, parece ser interessante. Outro problema mais envolvente seria a questão de estabilidade/instabilidade das soluções de equilíbrio. Da teoria desenvolvida em Henry, [23], observamos que para obter um resultado deste tipo para soluções de equilíbrio da a Eq. (6.8) basta analizar as propriedades espectrais do operador linear

$$
\mathcal{L}(g)=\frac{d^{2} g}{d x^{2}}+w g+P^{\prime}(a) g
$$

Neste caso, a teoria desenvolvida no capítulo 3 será essencial.

5. Outro tipo de problema que pode ser naturalmente abordado é o da existência e estabilidade de soluções de equilíbrio para modelos mais gerais de tipo parabólico

$$
u_{t}=u_{x x}+Z \delta(x) u+w u+P(u)
$$


$\operatorname{com}(x, t) \in \mathbb{R} \times \mathbb{R}^{+}, u(x, t) \in \mathbb{R}, w \in \mathbb{R}$ um parâmetro, $P$ um polinômio com coeficientes reais de grau maior a um e $\delta$ é a medida de Dirac centrada no ponto $x=0$. Note que neste caso a equação (6.10) não tem nenhum tipo de simetria básica. 



\section{APÊNDICE A}

¿ PROVA DA ESTABILIDADE PARA A EQUAÇÃO CQ-S

No presente apêndice apresentaremos uma idéia idéia da prova do Teorema 3.1.1 enunciado no capítulo 3 sobre a estabilidade orbital de standing waves para a CQ-S

$$
i u_{t}+u_{x x}+u\left(|u|^{2}+|u|^{4}\right)=0
$$

com $u: \mathbb{R} \times \mathbb{R} \rightarrow \mathbb{C}$, para $u=u(x, t)$. As idéias aqui apresentadas fazem parte da teoria que foi desenvolvida por Grillakis\&Shatah\&Straus em [27]-[30]. Desta maneira, se substituimos $u=R(u)+i \operatorname{Im}(u)=\alpha+i \beta$ na equação (A.1), obtemos o seguinte sistema de equações diferenciais parciais

$$
\left\{\begin{array}{l}
\alpha_{t}=-\beta_{x x}-\beta\left(\alpha^{2}+\beta^{2}\right)-\beta\left(\alpha^{2}+\beta^{2}\right)^{2} \\
\beta_{t}=\alpha_{x x}+\alpha\left(\alpha^{2}+\beta^{2}\right)+\alpha\left(\alpha^{2}+\beta^{2}\right)^{2}
\end{array},\right.
$$

que pode ser escrito na forma matricial

$$
\frac{d}{d t}\left[\begin{array}{l}
\alpha \\
\beta
\end{array}\right]=\left[\begin{array}{cc}
0 & 1 \\
-1 & 0
\end{array}\right]\left[\begin{array}{l}
-\alpha_{x x}-\alpha\left(\alpha^{2}+\beta^{2}\right)-\alpha\left(\alpha^{2}+\beta^{2}\right)^{2} \\
-\beta_{x x}-\beta\left(\alpha^{2}+\beta^{2}\right)-\beta\left(\alpha^{2}+\beta^{2}\right)^{2}
\end{array}\right] .
$$

Se definimos $J$ como

$$
J=\left[\begin{array}{cc}
0 & 1 \\
-1 & 0
\end{array}\right]
$$


e $T(s)=e^{J s}$, para $s \in \mathbb{R}$, isto é

$$
T(s)=\left[\begin{array}{cc}
\cos (s) & -\sin (s) \\
\sin (s) & \cos (s)
\end{array}\right]
$$

observamos que a equação (A.3) apresenta simetrias de rotação, ou seja, se $[\alpha, \beta]^{t}$ é solução de (A.3) então $T(s)[\alpha, \beta]^{t}$ é também solução de (A.3) para todo $s \in \mathbb{R}$. Da mesma forma se $[\alpha, \beta]^{t}$ é solução de (A.3) então

$$
S(r)\left[\begin{array}{l}
\alpha \\
\beta
\end{array}\right](x, t)=\left[\begin{array}{l}
\alpha \\
\beta
\end{array}\right](x+r, t),
$$

é também solução de (A.3) para todo $r \in \mathbb{R}$. Isto significa que a equação (A.3) presenta simetrias de translação. Por outro lado, as soluções do tipo standing waves nesta notação tem a seguinte forma

$$
[\alpha, \beta]^{t}(x, t)=T(-w t) \mathbf{A}_{w}^{t}(x)
$$

onde $\mathbf{A}_{w}(x):=\left[a_{w}(x), 0\right]$. Substituindo (A.7) em (A.3), deduzimos facilmente que $a_{w}$ satisfaz a seguinte equação diferencial ordinária

$$
a_{w}^{\prime \prime}+w a+a^{3}+a^{5}=0
$$

Agora seja $X=H_{p e r}^{1}([0, L])$. Para $[\alpha, \beta] \in X \times X$ definimos os funcionais $E, F$ : $X \times X \rightarrow \mathbb{R}$, por

$$
\begin{aligned}
& E\left([\alpha, \beta]^{t}\right)=\frac{1}{2} \int_{0}^{L} \alpha_{x}^{2}+\beta_{x}^{2}-\frac{1}{2}\left[\alpha^{4}+2 \alpha^{2} \beta^{2}+\beta^{4}\right]-\frac{1}{3}\left[\alpha^{6}+3 \alpha^{4} \beta^{2}+3 \alpha^{2} \beta^{4}+\beta^{6}\right] d x, \\
& F\left([\alpha, \beta]^{t}\right)=\frac{1}{2} \int_{0}^{L} \alpha^{2}+\beta^{2} d x .
\end{aligned}
$$

Notamos que os funcionais $E, F$ são quantidades conservadas para a equação (A.3) e invariantes pelos grupos $T$ e $S$ definidos em (A.5) e (A.6) respectivamente. As derivadas dos funcionais $E, F$, são dados por

$$
E^{\prime}\left([\alpha, \beta]^{t}\right)=\left[\begin{array}{c}
-\alpha_{x x}-\alpha\left(\alpha^{2}+\beta^{2}\right)-\alpha\left(\alpha^{2}+\beta^{2}\right)^{2} \\
-\beta_{x x}-\beta\left(\alpha^{2}+\beta^{2}\right)-\beta\left(\alpha^{2}+\beta^{2}\right)^{2}
\end{array}\right],
$$


e

$$
\begin{aligned}
& E^{\prime \prime}\left([\alpha, \beta]^{t}\right)= \\
& {\left[\begin{array}{cc}
-\frac{d^{2}}{d x^{2}}-3 \alpha^{2}-\beta^{2}-5 \alpha^{4}-6 \alpha^{2} \beta^{2}-\beta^{4} & -2 \alpha \beta-4 \alpha^{3} \beta-4 \alpha \beta^{3} \\
-2 \alpha \beta-4 \alpha^{3} \beta-4 \alpha \beta^{3} & -\frac{d^{2}}{d x^{2}}-3 \beta^{2}-\alpha^{2}-5 \beta^{4}-6 \alpha^{2} \beta^{2}-\alpha^{4}
\end{array}\right] .}
\end{aligned}
$$

Da mesma forma para $F$ temos que

$$
F^{\prime}\left([\alpha, \beta]^{t}\right)=[\alpha, \beta]^{t}
$$

e

$$
F^{\prime \prime}\left([\alpha, \beta]^{t}\right)=\left[\begin{array}{ll}
1 & 0 \\
0 & 1
\end{array}\right] .
$$

Comparando (A.3) com (A.10), observamos que (A.3) pode ser escrita na forma Hamiltoniana

$$
\frac{d \mathbf{u}}{d t}=J E^{\prime}(\mathbf{u}(t))
$$

aqui $\mathbf{u}=[\alpha, \beta]^{t}$. Seja agora $\Psi: X \times X \rightarrow \mathbb{R}$, definida por

$$
\Psi\left([\alpha, \beta]^{t}\right)=E\left([\alpha, \beta]^{t}\right)-w F\left([\alpha, \beta]^{t}\right),
$$

então é facil ver que

$$
\Psi^{\prime}\left(\mathbf{A}_{w}^{t}\right)=\left[-a_{w}^{\prime \prime}-a_{w}^{3}-a_{w}^{5}-w a_{w}, 0\right]^{t}=[0,0]^{t}
$$

isto é, $\mathbf{A}_{w}^{t}$ é um ponto crítico do funcional $\Psi$. Agora para nosso estudo de estabilidade, o funcional $\Psi$ será estimado em termos de $\rho\left(\mathbf{u}(t), \Omega_{\mathbf{A}_{w}}\right)$ e será usado para medir a divergência de $\mathbf{u}(t)$ da órbita $\Omega_{\mathbf{A}_{w}}$ (ver Definição 3.1.1). Para isto vamos considerar (com base nas simétrias da equação (A.3)) a seguinte variável

$$
\left[\phi_{1}, \phi_{2}\right]^{t} \equiv T(\theta) \mathbf{u}(x-s, t)-\mathbf{A}_{w}^{t},
$$


então, por conservação das quantidades (A.9), (A.14) e o Teorema de Taylor temos que

$$
\begin{aligned}
\Delta \Psi & \equiv \Psi(\mathbf{u}(\cdot, 0))-\Psi\left(\mathbf{A}_{w}^{t}(\cdot)\right) \\
& =\Psi(\mathbf{u}(\cdot, t))-\Psi\left(\mathbf{A}_{w}^{t}(\cdot)\right) \\
& =\Psi(T(\theta) \mathbf{u}(\cdot-s, t))-\Psi\left(\mathbf{A}_{w}^{t}(\cdot)\right) \\
& =\Psi\left(\mathbf{A}_{w}^{t}+\left[\phi_{1}, \phi_{2}\right]^{t}\right)-\Psi\left(\mathbf{A}_{w}^{t}\right) \\
& =\left\langle\Psi^{\prime \prime}\left(\mathbf{A}_{w}^{t}\right)\left[\phi_{1}, \phi_{2}\right]^{t},\left[\phi_{1}, \phi_{2}\right]^{t}\right\rangle+o\left(\left\|\left[\phi_{1}, \phi_{2}\right]^{t}\right\|_{1}^{2}\right) .
\end{aligned}
$$

Das fórmulas obtidas anteriormente para $E^{\prime \prime}$ e $F^{\prime \prime}$ deduzimos que o operador linear $\Psi^{\prime \prime}\left(\mathbf{A}_{w}^{t}\right): X \times X \rightarrow X^{*} \times X^{*}$, vem dado pelo operador diagonal:

$$
\Psi^{\prime \prime}\left(\left[a_{w}, 0\right]^{t}\right)=\left[\begin{array}{cc}
\mathcal{L}_{R} & 0 \\
0 & \mathcal{L}_{\text {Im }}
\end{array}\right],
$$

com $\mathcal{L}_{R}$ e $\mathcal{L}_{\text {Im }}$ dados em (4.83). Em particular a forma quadrática

$$
\left\langle\Psi^{\prime \prime}\left(\mathbf{A}_{w}^{t}\right)\left[\phi_{1}, \phi_{2}\right]^{t},\left[\phi_{1}, \phi_{2}\right]^{t}\right\rangle,
$$

em (A.16), vem dada por

$$
\left\langle\Psi^{\prime \prime}\left(\mathbf{A}_{w}^{t}\right)\left[\phi_{1}, \phi_{2}\right]^{t},\left[\phi_{1}, \phi_{2}\right]^{t}\right\rangle=\left\langle\mathcal{L}_{R} \phi_{1}, \phi_{1}\right\rangle+\left\langle\mathcal{L}_{I m} \phi_{2}, \phi_{2}\right\rangle
$$

Por outro lado, definindo $M_{w} \subset X \times X$, por

$$
M_{w}:=\left\{\mathbf{h} \in X \times X \mid F(\mathbf{h})=F\left(\mathbf{A}_{w}^{t}\right)\right\}
$$

com $F$ definido em (A.9). Como $F$ é um funcional suave definido sobre $X \times X$ e de posto um sobre $M_{w}$, então $M_{w}$ é uma variedade infinito dimensional de codimensão um que contem a órbita $T(\theta) \mathbf{A}_{w}^{t}$ gerada pelo grupo $T$. O espaço tangente a $M_{w}$ em $\mathbf{A}_{w}^{t}$ esta dado por,

$$
\begin{aligned}
T_{\mathbf{A}_{w}} M_{w} & :=\left\{\mathbf{h}=\left[h_{1}, h_{2}\right]^{t} \in X \times X \mid\left\langle\mathbf{h}, F^{\prime}\left(\mathbf{A}_{w}^{t}\right)\right\rangle=0\right\}, \\
& =\left\{\left[h_{1}, h_{2}\right]^{t} \in X \times X \mid\left\langle h_{1}, a_{w}\right\rangle=0\right\} .
\end{aligned}
$$

O objetivo agora será mostrar que o operador $\Psi^{\prime \prime}\left(\mathbf{A}_{w}^{t}\right)$ é não negativo sobre o espaço $T_{\mathbf{A}_{w}} M_{w}$. Da equação (4.80), temos que $E^{\prime}\left(\mathbf{A}_{w}^{t}\right)=w F^{\prime}\left(\mathbf{A}_{w}^{t}\right)$, e assim derivando esta 
igualdade respeito da variável $w$, obtemos,

$$
\Psi^{\prime \prime}\left(\mathbf{A}_{w}^{t}\right) \frac{\partial}{\partial w} \mathbf{A}_{w}^{t}=F^{\prime}\left(\mathbf{A}_{w}^{t}\right)=\left[a_{w}, 0\right]^{t}
$$

Agora, se definimos $d:(-\infty, f(L)) \rightarrow \mathbb{R}$, por

$$
d(w)=\Psi\left(\mathbf{A}_{w}^{t}\right)=E\left(\mathbf{A}_{w}^{t}\right)-w F\left(\mathbf{A}_{w}^{t}\right)
$$

com $f(L)$ dado em (4.66) e as funções $E, F$, dadas em (A.9), temos que

$$
d^{\prime \prime}(w)=-\left\langle F^{\prime}\left(\mathbf{A}_{w}^{t}\right), \frac{\partial}{\partial w} \mathbf{A}_{w}^{t}\right\rangle=-\frac{d}{d w} \int_{0}^{L} a_{w}^{2}(x) d x,
$$

logo de (A.20), (A.22) e a convexidade da função $d$ mostrada na seção 4.4 do capítulo 4, deduzimos que

$$
d^{\prime \prime}(w)=-\left\langle\Psi^{\prime \prime}\left(\mathbf{A}_{w}^{t}\right) \frac{\partial}{\partial w} \mathbf{A}_{w}^{t}, \frac{\partial}{\partial w} \mathbf{A}_{w}^{t}\right\rangle>0 .
$$

Por outro lado dos Teoremas 4.3.2, 4.3.1 e a expressão (A.17), obtemos as seguintes propriedades espectrais para $\Psi^{\prime \prime}\left(\mathbf{A}_{w}^{t}\right)$

1. $\Psi^{\prime \prime}\left(\mathbf{A}_{w}^{t}\right)$ tem um único autovalor negativo $-\lambda_{1}^{2}$, com auto-função $\left[\chi_{1}, 0\right]^{t}$, onde $\mathcal{L}_{R} \chi_{1}=-\lambda_{1}^{2} \chi_{1}$

2. O núcleo do operador $\Psi^{\prime \prime}\left(\mathbf{A}_{w}^{t}\right)$ tem dimensão dois, uma base para o núcleo deste operador é dada pelo conjunto $\left\{\left[a_{w}^{\prime}, 0\right]^{t},\left[0, a_{w}\right]^{t}\right\}$.

3. O resto do espectro é uma sequência de autovalores positivos $\left\{r_{i}\right\}_{i=0}^{\infty}$ satisfazendo $\lim _{i \rightarrow \infty} r_{i}=\infty$. (ver Teoremas 4.3.2 e 4.3.1).

Agora se definimos $\mathcal{P}$, por

$$
\mathcal{P}=\left\{[p, q]^{t} \in X \times X \mid\left\langle p, a_{w}\right\rangle=0,\left\langle q, a_{w}\right\rangle=0 .\left\langle p, a_{w}^{\prime}\right\rangle=0\right\}
$$

A saber, $[p, q]^{t} \in \mathcal{P} \Leftrightarrow[p, q]^{t} \perp \operatorname{Ker}\left(\Psi^{\prime \prime}\left(\mathbf{A}_{w}^{t}\right)\right)$ e $[p, q]^{t} \in T_{\mathbf{A}_{w}} M_{w}$, então temos o seguinte lema.

Lema A.0.10. Se $\left[y_{1}, y_{2}\right]^{t} \in \mathcal{P}$, então $\left\langle\Psi^{\prime \prime}\left(\boldsymbol{A}_{w}^{t}\right)\left[y_{1}, y_{2}\right]^{t},\left[y_{1}, y_{2}\right]^{t}\right\rangle>0$. 
Demonstração. Da equação (A.23) obtemos que $\left\langle\Psi^{\prime \prime}\left(\mathbf{A}_{w}^{t}\right) \frac{\partial}{\partial w} \mathbf{A}_{w}^{t}, \frac{\partial}{\partial w} \mathbf{A}_{w}^{t}\right\rangle<0$, logo tomando a decomposição espectral ortogonal de $\frac{\partial}{\partial w} \mathbf{A}_{w}^{t}$, isto é,

$$
\frac{\partial}{\partial w} \mathbf{A}_{w}^{t}=\alpha_{0}\left[\chi_{1}, 0\right]^{t}+\alpha_{1}\left[a^{\prime}, 0\right]^{t}+\alpha_{2}[0, a]^{t}+\alpha_{3}\left[p_{0}, q_{0}\right]^{t}
$$

onde $\left[p_{0}, q_{0}\right]^{t}$ satisfaz $\left\langle\Psi^{\prime \prime}\left(\mathbf{A}_{w}^{t}\right)\left[p_{0}, q_{0}\right]^{t},\left[p_{0}, q_{0}\right]^{t}\right\rangle \geq c_{0}\left\|\left[p_{0}, q_{0}\right]^{t}\right\|^{2}$, obtemos que

$$
\left\langle\Psi^{\prime \prime}\left(\mathbf{A}_{w}^{t}\right) \frac{\partial}{\partial w} \mathbf{A}_{w}^{t}, \frac{\partial}{\partial w} \mathbf{A}_{w}^{t}\right\rangle=-\alpha_{0}^{2} \lambda_{1}^{2}+\left\langle\Psi^{\prime \prime}\left(\mathbf{A}_{w}^{t}\right)\left[p_{0}, q_{0}\right]^{t},\left[p_{0}, q_{0}\right]^{t}\right\rangle<0 .
$$

Suponha agora que $\left[y_{1}, y_{2}\right]^{t} \in \mathcal{P}$, então

$$
\left[y_{1}, y_{2}\right]=\alpha\left[\chi_{1}, 0\right]+[p, q]
$$

com $[p, q]^{t}$ no espaço positivo associado a $\Psi^{\prime \prime}\left(\mathbf{A}_{w}^{t}\right)$. Como $\Psi^{\prime \prime}\left(\mathbf{A}_{w}^{t}\right) \frac{\partial}{\partial w} \mathbf{A}_{w}^{t}=[a, 0]^{t}$, então deduzimos que

$$
0=\left\langle\Psi^{\prime \prime}\left(\mathbf{A}_{w}^{t}\right) \frac{\partial}{\partial w} \mathbf{A}_{w}^{t},[p, q]^{t}\right\rangle=-\alpha_{0} \alpha \lambda^{2}+\left\langle\Psi^{\prime \prime}\left(\mathbf{A}_{w}^{t}\right)\left[p_{0}, q_{0}\right]^{t},[p, q]^{t}\right\rangle .
$$

Pela desigualdade de Cauchy-Schwarz para formas sesquilineares temos

$$
\begin{aligned}
\left\langle\Psi^{\prime \prime}\left(\mathbf{A}_{w}^{t}\right)\left[y_{1}, y_{2}\right]^{t},\left[y_{1}, y_{2}\right]^{t}\right\rangle & =-\alpha^{2} \lambda^{2}+\left\langle\Psi^{\prime \prime}\left(\mathbf{A}_{w}^{t}\right)[p, q]^{t},[p, q]^{t}\right\rangle \\
& \geq-\alpha^{2} \lambda^{2}+\frac{\left\langle\Psi^{\prime \prime}\left(\mathbf{A}_{w}^{t}\right)[p, q]^{t},\left[p_{0}, q_{0}\right]^{t}\right\rangle^{2}}{\left\langle\Psi^{\prime \prime}\left(\mathbf{A}_{w}^{t}\right)\left[p_{0}, q_{0}\right]^{t},\left[p_{0}, q_{0}\right]^{t}\right\rangle} \\
& >-\alpha^{2} \lambda^{2}+\frac{\left(\alpha_{0} \alpha \lambda^{2}\right)^{2}}{\alpha_{0}^{2} \lambda^{2}}=0,
\end{aligned}
$$

como queríamos provar.

Finalmente, seguindo as idéias do Teorema 3.4 em [27] e do Lema 7.8 em [8], pode-se obter constantes $c_{1}>0, \delta_{1}>0$, tal que,

$$
E(\mathbf{u}(t))-E\left(\mathbf{A}_{w}^{t}\right)=E(\mathbf{u})-E\left(\mathbf{A}_{w}^{t}\right) \geq c_{1} \rho\left(\mathbf{u}, \Omega_{\mathbf{A}_{w}}\right)
$$

para todo $\mathbf{u} \in M_{w} \operatorname{com} \rho\left(\mathbf{u}, \Omega_{\mathbf{A}_{w}}\right)<\delta_{1}$. Então a estabilidade segue da continuidade dos funcionais $E, F$, do fluxo $t \rightarrow u(t)$ e de um argumento por contradição baseado em (A.26). Isto finaliza a prova do Teorema 3.1.1. 


\section{APÊNDICE B}

\section{¿DINÂMICA DA EQUAÇÃO DIFERENCIAL ORDINÁRIA

$$
A^{\prime \prime}+R A+S A^{3}+T A^{5}=0
$$

Conforme foi visto nos Capítulos (4)-(5), a equação diferencial ordinária não linear,

$$
a^{\prime \prime}+r a+s a^{3}+t a^{5}=0
$$

desenpenha um papel muito importante no estudo sobre a existência de diferentes tipos de soluções ondas viajantes para as equações CQ-S (4.1) (caso periódico e não periódico), MC-KdV em (4.121) e CQD-S em (5.2). É por isto que neste apêndice vamos estudar desde o ponto de vista qualitativo o comportamento das soluções da equação (B.1), para diferentes valores dos parametros $r, s, t \in \mathbb{R}$. Este análise seguramente vai dar para nos uma idéia de onde procurar outras soluções relevantes para Eq. (B.1).

Observamos primeiramente que a equação (B.1) admite uma integral primeira $H$ : $\mathbb{R}^{2} \rightarrow \mathbb{R}$, definida por:

$$
H[x, y]=\frac{y^{2}}{2}+r \frac{x^{2}}{2}+s \frac{x^{4}}{4}+t \frac{x^{6}}{6}
$$

isto quer dizer que as curvas de nível da função $H$, coincidem com as trajetórias das curvas $\gamma(t):=\left[a(t), a^{\prime}(t)\right]=:[x(t), y(t)] \in \mathbb{R}^{2}$ no plano $x$-y que são soluções do sistema 
Hamiltoniano:

$$
\left\{\begin{array}{l}
x^{\prime}=y \\
y^{\prime}=-r x-s x^{3}-t x^{5},
\end{array}\right.
$$

que é equivalente a equação (B.1). Logo, para um total entendimento das soluções associadas a equação (B.1) basta conhecer geometricamente as curvas de nível da função $H$.

Antes de comecar nosso análise, daremos as formulas dos pontos de equilíbrio do sistema (B.3). Os pontos de equilíbrio do sistema (B.3) estão ubicados sobre o eixo $x(y=0)$ e são dados pelas seguintes expressões:

$$
\begin{aligned}
& x_{0}=0, \\
& x_{+}^{2}=\frac{-s+\sqrt{s^{2}-4 r t}}{2 t}, \\
& x_{-}^{2}=\frac{-s-\sqrt{s^{2}-4 r t}}{2 t} .
\end{aligned}
$$

Com $x_{+}^{2}, x_{-}^{2}$, sendo os quadrados das raizes do polinômio

$$
q(x):=r+s x^{2}+t x^{4}
$$

Notamos de (B.4) que o ponto $(x, y)=(0,0)$ é sempre um ponto de equilíbrio do sistema (B.3), sem importar o valor dos parâmetros $r, s, t$.

Por outro lado, sabemos que a quantidade de pontos críticos e porém a dinâmica da equação (B.1) vai depender entre outras coisas do sinal da quantidade $\Delta:=s^{2}-4 r t$. Assim, consideramos 3 casos:

I- $\Delta<0$. Neste caso, de (B.4) deduzimos que o polinômio $q$ em (B.5) tem 4 raízes complexas, logo o sistema (B.3) neste caso tem exatamente um único ponto de equilíbrio, a saber o ponto $(x, y)=(0,0)$. Notamos também que do fato que $s^{2}<4 r t$ deduzimos que os parâmetros $r$ e $t$ devem ter o mesmo sinal. Por exemplo, quando $r=-1, s=1$ e $t=-1$ temos o seguinte gráfico, para os niveis $H[\cdot, \cdot]=-1, H[\cdot, \cdot]=0$ e $H[\cdot, \cdot]=1$ 


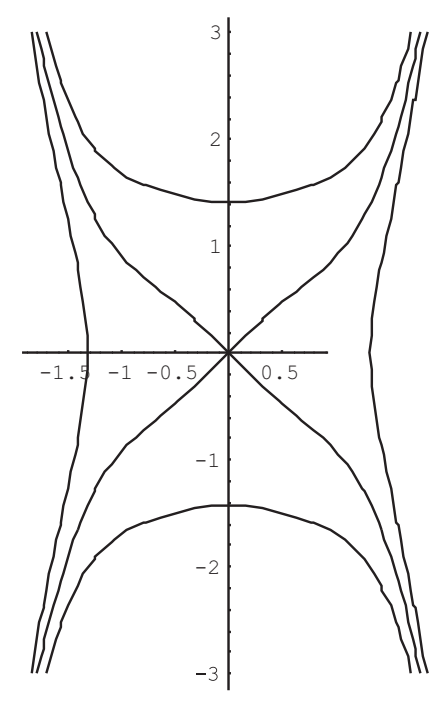

Aqui o ponto crítico $(0,0)$ é um ponto de sela.

Agora se $r=1, s=-1$ e $t=1$ temos o seguinte gráfico, para os niveis $H[\cdot, \cdot]=$ $0.2, H[\cdot, \cdot]=2$ que representão soluções periódicas que mudam de sinal

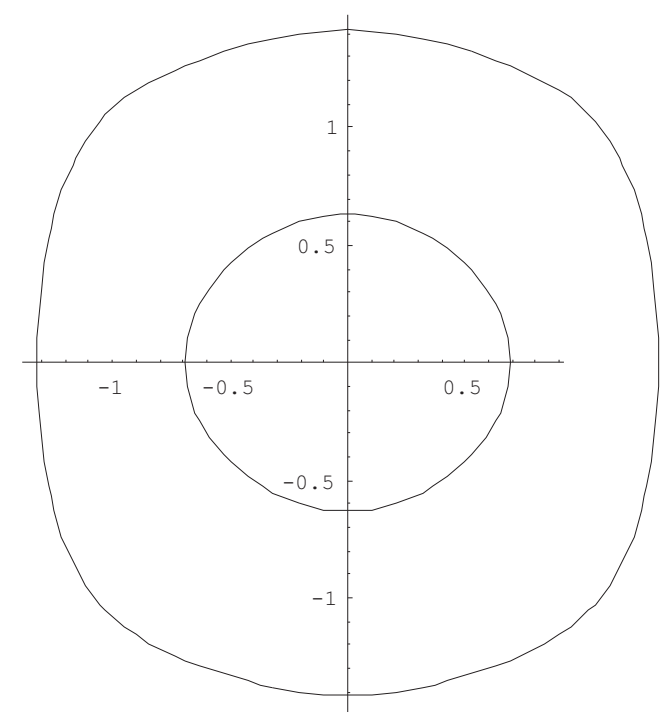

Aqui o ponto de equilíbrio $(0,0)$ é um centro.

Observação B.0.6. 1- O gráfico das curvas de nível da função $H$ no caso em que $\Delta<0, r<0, s<0$ e $t<0$ é semelhante ao apresentado no primeiro gráfico do item I. Em particular o ponto $(0,0)$ contínua sendo uma sela. 
2- O gráfico das curvas de nível da função $H$ no caso em que $\Delta<0, r>0$, $s>0$ e $t>0$ é semelhante ao apresentado no segundo gráfico do item I. Em particular o ponto $(0,0)$ contínua sendo uma centro.

Concluimos disto que a mudançã no sinal do parâmetro $s$ quando $\Delta<0$, não determina uma mudançã radical nas soluções do sistema (B.3).

II- $\Delta=0$. Neste caso, $x_{+}^{2}=x_{-}^{2}=\frac{-s}{2 t}$. Assim, o polinômio $q$ dado em (B.5) tem duas raízes de multiplicidade algébrica 2 . No caso que st $>0$, ditas raízes são complexas. Já para st $<0$ temos a existência de duas raízes reais de multiplicidade algébrica 2. E assim, neste caso teriamos dois pontos de equilíbrio degenerados, isto é, a matriz resultante da linearização em ditos pontos tem o zero 0 como autovalor.

O estudo das curvas de nível da função $H$ neste caso $(\Delta=0)$ é semelhante ao caso $\Delta<0$. Em particular os parâmetros $r$ e $t$ devem ter o mesmo sinal. Quando $r<0$ e $t<0$ o ponto $(0,0)$ é uma sela (ver a primeira figura do item $I$ ). Quando $r>0$ e $t>0$ o ponto $(0,0)$ é um centro (ver a segunda figura do item $I$ ).

III- $\Delta>0$. A dinâmica da equação (B.1) neste caso é muito mais rica. Em particular a quantidade de pontos de equilíbrio muda da seguinte maneira:

\section{(a) 5 pontos de equilíbrio.}

i. $r>0, s<0$ e $t>0$. Por exemplo quando $r=1, s=-4$ e $t=$ 1 temos os seguientes pontos de equilíbrio $(-1.93185,0),(-0.5176,0)$, $(0,0),(0.5176,0)$ e $(1.93185,0)$. Estos pontos de equilíbrio são centro, sela, centro sela e centro respectivamente. Neste caso o nível $H[\cdot, \cdot]=c$ com $c \approx 0.0661$ denota 4 tipos diferentes de soluções não periódicas que são naturalmente separadas pelos pontos de equilíbrio. O seguinte gráfico, mostra dito comportamento: 


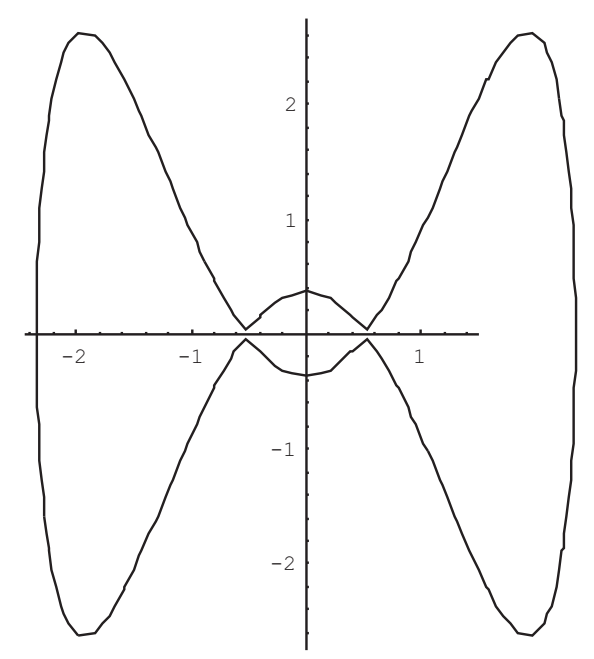

Além disso, vemos que esta curva divide a dinâmica do sistema (B.3) de uma maneira particular, por exemplo pra os niveis $H[\cdot, \cdot]=c$ com $c=0.05, c=0.9$ e $c=-2.5$ obtemos diferentes tipos de soluções periódicas:

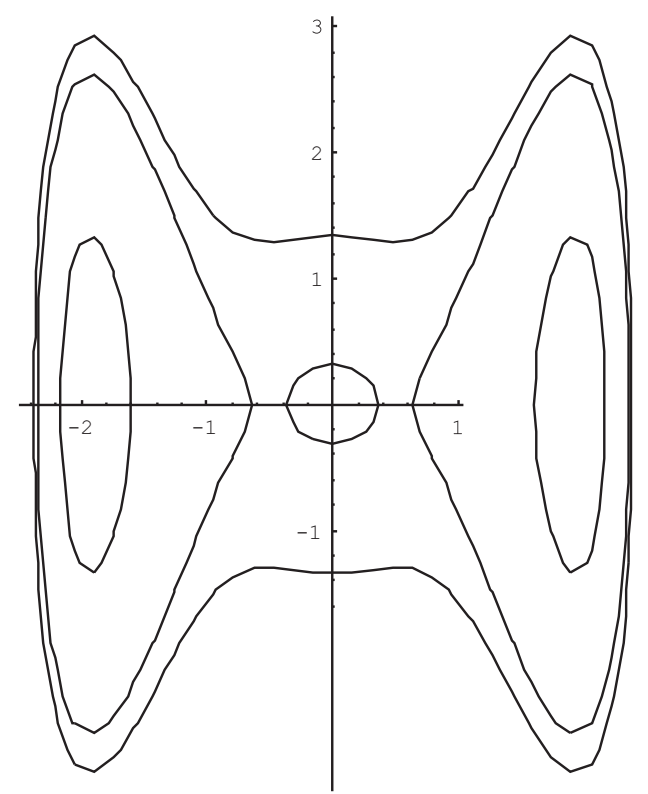

Observamos que em particular o nível $H[\cdot, \cdot]=0.05$, da origem a três tipos de soluções periódicas: uma negativa, uma que muda de sinal e uma positiva, o nível $H[\cdot, \cdot]=0.9$ é a curva exterior e denota soluções que mudam de sinal e o nível $H[\cdot, \cdot]=-2.5$, denota dos tipos de soluções periódicas uma positiva e uma negativa. 
ii. $r<0, s>0$ e $t<0$. Por exemplo quando $r=-1, s=4$ e $t=-1$ temos os seguientes pontos de equilíbrio $(-1.93185,0),(-0.5176,0),(0,0)$, $(0.5176,0)$ e $(1.93185,0)$. Neste caso os pontos de equilíbrio são sela, centro, sela, centro e sela respectivamente. Neste caso o nível $H[\cdot, \cdot]=c$ com $c=3.382$ denota 4 tipos diferentes de soluções não periódicas que são naturalmente separadas pelos pontos de equilíbrio $(-1.93185,0)$ e $(1.93185,0)$. Já o nível $c=0$ denota uma curva que passa pelo origen $(0,0)$ que representa 2 tipos de soluções não periódicas: uma positiva e uma negativa que se aproxima do equilíbrio $(0,0)$ no infinito. O seguinte gráfico da uma idéia de dito comportamento:

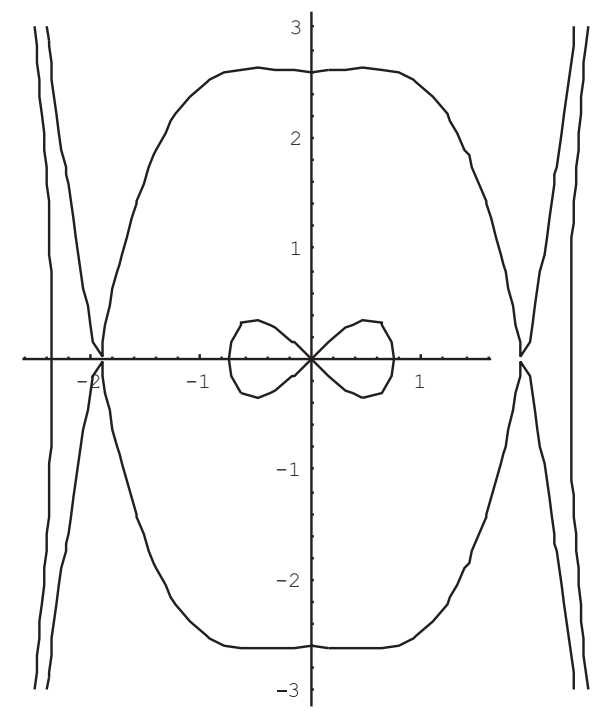

Outro tipo de soluções aparecem no seguinte gráfico: 


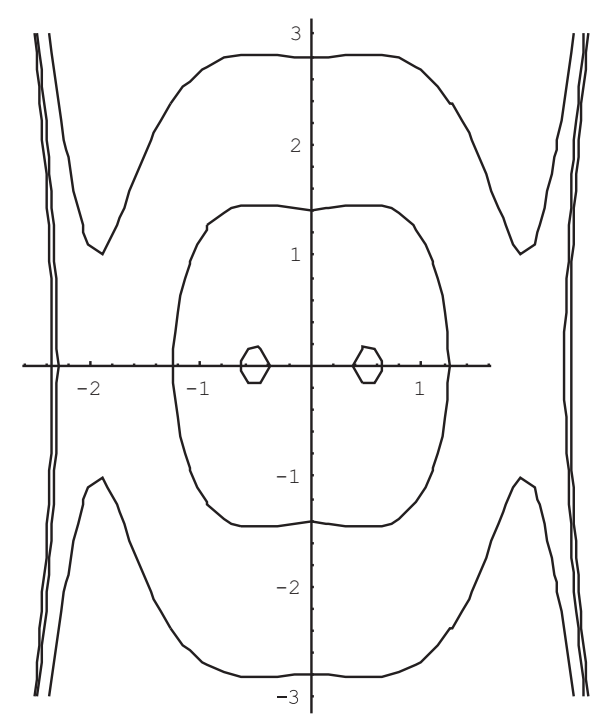

Em particular o nível $c=-0.05$ representa duas soluções periódicas uma positiva que tem o ponto de equilíbrio $(0.5176,0)$ no seu interior e uma negativa que tem o ponto de equilíbrio $(-0.5176,0)$ no seu interior. Já o nível $c=1$ representa uma solução periódica que muda de sinal. Outro tipo de solução aparece quando $H[\cdot, \cdot]=3.9$.

\section{(b) 3 pontos de equilíbrio.}

i. $r>0, s>0$ e $t<0$. Por exemplo quando $r=1, s=4$ e $t=-1$ temos os seguientes pontos de equilíbrio $(-2.05817,0),(0,0)$ e $(2.05817,0)$, Neste caso os pontos de equilíbrio são classificados como sela, centro e sela respectivamente. Neste caso o nível $H[\cdot, \cdot]=1$ denota uma solução periódica que muda de sinal. Já o nível $H[\cdot, \cdot]=7.35$ (a curva fechada e exterior no gráfico abaixo) denota 4 tipos diferentes de soluções não periódicas que são naturalmente separadas pelos pontos de equilíbrio $(-2.05817,0)$ e $(2.05817,0)$. O seguinte gráfico mostra o comportamento descrito anteriormente 


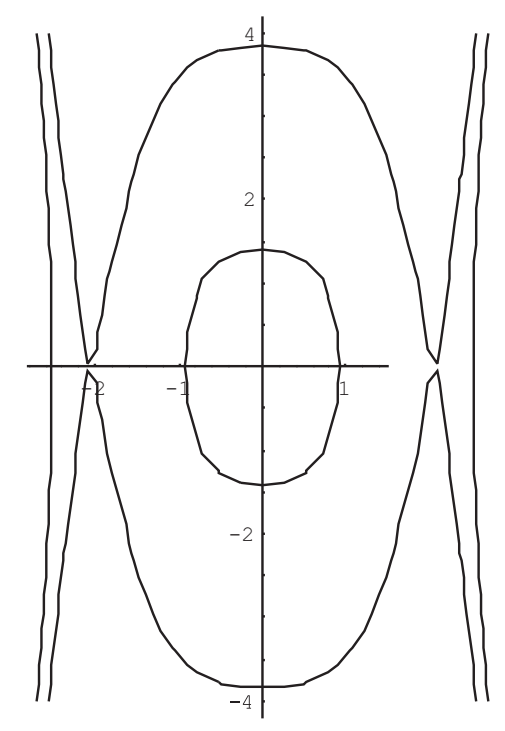

ii. $r>0, s \leq 0$ e $t<0$. Neste caso o comportamento das curvas de nível da função $H$ é semelhante ao caso imediatamente anterior. Notamos que o sinal do parâmetro $s$ não altera o comportameto qualitativo das soluções associadas com o sistema (B.3).

iii. $r<0, s>0$ e $t>0$. Por exemplo quando $r=-1, s=1$ e $t=1$ temos os seguintes pontos de equilíbrio $(-0.7861,0),(0,0)$ e $(-0.7861,0)$ que são classificados como centro, sela e centro respectivamente. O seguinte gráfico é o retrato de fase para a equação (B.1) neste caso

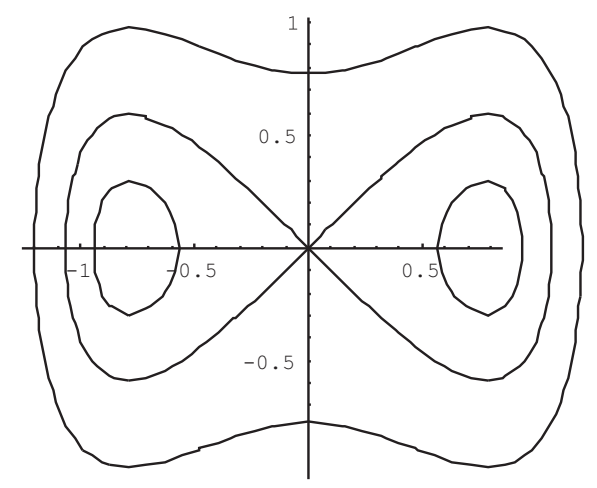

aqui a curva exterior é o nível $H[\cdot, \cdot]=0.3$ a qual denota soluções periódicas que mudam de sinal. A curva do medio é o nível $H[\cdot, \cdot]=0$ e denota soluções com um sinal definido: positivo ou negativo e não 
periódicas. Estas podem ser escritas analíticamente na forma:

$$
a(x)= \pm\left[\frac{1}{4}+\frac{\sqrt{57}}{12} \cosh (2 x)\right]^{-\frac{1}{2}}
$$

(ver formula (4.64)).

A curva interior é o nível $H[\cdot, \cdot]=-0.13$ e denota soluções periódicas da equação (B.1) com un sinal definido: positivo ou negativo. As soluções periódicas positivas foram estudadas no capítulo 4. Lembramos aqui que elas são dadas analíticamente pela formula (4.55).

iv. $r<0, s \leq 0$ e $t>0$. Neste caso o comportamento das curvas de nível da função $H$ é semelhante ao caso imediatamente anterior. Como antes (ver item (b)-ii) vemos que o sinal do parâmetro $s$ não altera o comportameto qualitativo das soluções associadas com o sistema (B.3).

(c) 1 ponto de equilíbrio.

i. $r<0, s<0$ e $t<0$. Neste caso o ponto $(0,0)$ é o único ponto de equilíbrio e é uma sela (ver o primeiro gráfico em I).

ii. $r>0, s>0$ e $t>0$. Neste caso o ponto $(0,0)$ é o único ponto de equilíbrio e é um centro (ver o segundo gráfico em II).

Isto conclue o análise qualitativo da equação (B.1). 
[1] J. P. Albert and J. L. Bona, Total positivity and the stability of internal waves in fluids of finite depth, IMA J. Applied Math. 46, 1-19, 1991.

[2] J. P. Albert, Positivity properties and stability of solitary-wave solutions of model equations for long waves, Comm. PDE. 17, 1-22, 1992.

[3] S. Albeverio, F. Gesztesy, R. Krohn, H. Holden, Solvable Models in quantum mechanics, AMS Chelsea publishing. 2004.

[4] J. Angulo, Non-Linear stability of periodic travelling-wave solutions for the Schrödinger and modified Korteweg-de Vries equation, J. Differential Equations, 1-30, 235, 2007.

[5] J. Angulo, F. Natali, Positivity properties of the Fourier transform and the stability of periodic travelling-wave solutions. SIAM J. Math. Anal., 1123-1151, 40, 2008.

[6] J. Angulo, Stability of dnoidal waves to Hirota-Satsuma system. Differential and Integral Equations, v. 18, no. 6, p. 611-645, 2005.

[7] J. Angulo, Stability of cnoidal waves for the Hirota-Satsuma system. Matemática Contemporanea, v. 27, p. 189-223, 2005. 
[8] J. Angulo, Nonlinear dispersive equations: Existence and stability of solitary and periodic travelling wave solutions, Mathematical Surveys and Monographs (SURV), 156, AMS, 2009.

[9] J. Angulo, J. Bona and M. Scialom, Stability of cnoidal waves, Advances in Differential Equations. 11, No.12, 1321-1374, 2006.

[10] J. Angulo and F.Linares, Periodic pulses of coupled nonlinear Schrödinger equations in optics. Indiana University Mathematics Journal, 56, no. 2, 847-877, 2007.

[11] J. Angulo and G. Ponce, The nonlinear Schrödinger equation with a periodic $\delta$-Interaction. pre-print, 2010.

[12] I. V. Barashenkov, A. D. Gocheva, V. G Makhankhov and I. V. Puzynin, Stability of the soliton like "bubbles", Physica. D, 240-254, 34, 1989.

[13] T. B. Benjamin, The stability of solitary waves. Proc. Roy. Soc. London A338, p. 153-183, 1972.

[14] T. B. Benjamin, Lectures on nonlinear wave motion. Nonlinear Wave Motion, A. C. Newell, ed., AMS, Providence, R. I., v. 15, p. 3-47, 1974.

[15] P. F. Byrd, M.D. Friedmann, Handbook of elliptical integrals for Enginners and Scientist, 2nd ed., Springer, NY, 1971.

[16] J. L. Bona, On the stability of solitary waves. Proc. Roy. Soc. London A344, p. 363-374, 1975.

[17] J. L. Bona and R. W. Smith, The initial-value problem for the Korteweg-de Vries equation. Philos. Trans. Royal Soc. London Series A 278, p. 555-601, 1975.

[18] J. L. Bona, P. Souganidis and W. Strauss, Stability and instability of solitary waves of Korteweg-de Vries type equations. Proc. Roy. Soc. London Ser. A411, p. 395-412, 1987. 
[19] T. Cazenave and F. B. Weissler, The Cauchy problem for the nonlinear Schrödinger in $H^{1}$, Manuscripta Math., 477-494, 61, 1988.

[20] T. Cazenave, Semilinear Schrödinger Equations, American Mathematical Society, (AMS). Lecture Notes, v. 10, 2003.

[21] E. A. Coddington and N. Levinson, Theory of Ordinary Differential Equations, Mc Graw Hill, New York, Toronto, London, 1955.

[22] M. Colin, M. Otha, Stability of solitary waves for nonlinear Schrödinger equation. Ann. Inst. H. Poincaré Anal. Nonlineaire, no. 5 753-764, 232006.

[23] D. Henry, Geometric Theory of Semilinear Parabolic Equation. Springer-Verlag. 1981.

[24] R. K. Dodd, J. C. Eilbeck, J. D. Gibbon and H. C. Morris, Solitons and Nonlinear Wave equations, Academic Press, London, 1982.

[25] R. Fukuizumi, and L. Jeanjean, Stability of standing waves for a nonlinear Schrödinger equation with a repulsive Dirac delta Potential, Discrete Contin. Dyn. Syst. 21, 2008.

[26] H. Goodman , P.J. Holmes and M. I. Weinstein, Strong NLS soliton-defect interaction, Physica D, 192, 215-248, 2004.

[27] M. Grillakis, J. Shatah, and W. Strauss, Stability theory of solitary waves in the presence of symmetry, I, J. Funct. Anal., 160-197, 74, 1987.

[28] M. Grillakis, Linearized instability for nonlinear Schrödinger and Klein-Gordon equations. Comm. Pure Appl. Math. 41, no. 6, 747-774, 1988.

[29] M. Grillakis, Analysis of the linearization around a critical point of an infinitedimensional Hamiltonian system. Comm. Puree Appl. Math., no. 3, 299-333, 43, 1990. 
[30] M. Grillakis, J. Shatah, and W. Strauss, Stability theory of solitary waves in the presence of symmetry, II, J. Funct. Anal., 308-348, 94, 1990.

[31] R.J. Jr. Iorio, V. Iorio de Magalhaes, Fourier Analysis and Partial Differential Equations, Cambridge Stud. Adv. Math., vol. 70, 2001.

[32] A. Jeffrey and T. Kakutani, Weak nonlinear dispersive waves: A discussion centered around the KdV equation. SIAM Rev., v. 14, p. 582-643, 1972.

[33] C. Jones, An instability mechanism for radially symmetric standing waves of a nonlinear Schrödiger equation. J. Differential Equations, no. 1, 34-62, 71, 1988.

[34] S. Karlin, Total Positivity, Stanford University Press, 1968.

[35] T. Kato, Perturbation Theory for Linear Operators. 2nd edition, Springer, Berlin, 1984 .

[36] D. J. Korteweg, G. de Vries, On the change of form of long waves advancing in a rectangular canal, and on a new type of long stationary waves. Philos. Mag. Ser., v. 39, no. 5, p. 422-443, 1895.

[37] E. Kuznetsov, A. Rubenchik and V. Zakharov, Soliton stability in plasma and hydro-dynamics. J. Phys., v. 142, p. 103-165, 1986.

[38] S. Le Coz, R. Fukuizumi, G. Fibich, B. Ksherim and Y. Sivan, Instability of bound states of a nonlinear Schrodinger equation with a Dirac Potential, Phys. D, 1103-1128, 237, 2008.

[39] W. Magnus and S. Winkler, Hill's Equation. Tracts in pure and Appl. Math., 20, Wesley. New York, 1976.

[40] W. Mio, T. Ogino, K. Minami, S. Takeda, Modified nonlinear Schrodinger equation for Alfvn waves propagating along the magnetic field in cold plasmas, J. Phys. Soc. Japan, 265-271, 41, 1976. 
[41] E. Mjolhus, On the modulational instability of hydromagnetic waves parallel to the magnetic field, J. Plasma Phys. 321-334, 16, 1976.

[42] A. Neves, Floquet's theorem and stability of periodic solitary waves. J. Dynam. Differential Equations, no. 3, 555-565, 21, 2009.

[43] M. Ohta, Stability and Instability of standing waves for one dimensional nonlinear Schrödinger equations with double power nonlinearity, Kodai Math. J., no. 1, 68-74, 18, 1995.

[44] F. Natali, J. Angulo. Stability an instability of periodic travelling wave solutions for the critical Korteweg-deVries and nonlinear Schrödinger equations. Physica D, v. 238, p. 603-621, 2009.

[45] S. Reed and B. Simon, Methods of modern mathematical Physics: Fourier Analysis Self-Adjointness, Academic Press, Vol. II, 1978.

[46] S. Reed and B. Simon, Methods of modern mathematical Physics: Analysis of Operators, Academic Press, Vol. IV, 1978.

[47] H. Takaoka, Well-posedness for the one-dimensional nonlinear Schrödinger with the derivative nonlinearity, Advances in Differential Equations, no.4, 561-580, 4, 1999.

[48] M. I. Weinstein, Liapunov stability of ground states of nonlinear dispersive evolution equations. Comm. Pure Appl. Math., v. 39, p. 51-68, 1986.

[49] M. I. Weinstein, Existence and dynamic stability of solitary wave solutions of equations arising in long wave propagation. Comm. PDE., v. 12, p. 1133-1173, 1987.

[50] M. I. Weinstein, Nonlinear Schrödinger equation and sharp interpolation estimates. Comm. Math. Phys., 87, p. 567-576, 1983. 
[51] V. E. Zakharov, Collapse of Langmuir waves. Sov. Phys. JETP., v. 35, p. 908-912, 1972. 